Linköping studies in science and technology. Thesis.

No. 1786

\title{
Parameter Estimation for Mobile Positioning Applications
}

\section{Parinaz Kasebzadeh}


This is a Swedish Licentiate's Thesis.

Swedish postgraduate education leads to a Doctor's degree and/or a Licentiate's degree.

A Doctor's Degree comprises 240 ECTS credits (4 years of full-time studies).

A Licentiate's degree comprises 120 ECTS credits,

of which at least 60 ECTS credits constitute a Licentiate's thesis.

Linköping studies in science and technology. Thesis.

No. 1786

\section{Parameter Estimation for Mobile Positioning Applications}

Parinaz Kasebzadeh

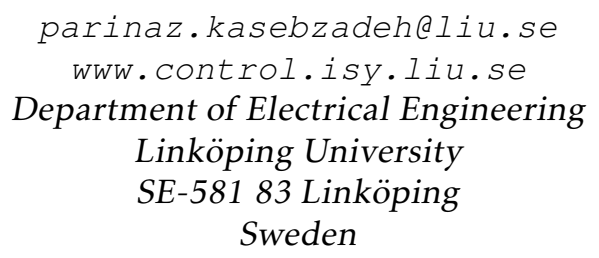

ISBN 978-91-7685-448-8

ISSN 0280-7971

Copyright (C) 2017 Parinaz Kasebzadeh

Printed by LiU-Tryck, Linköping, Sweden 2017 
To my beloved family and friends! 



\section{Abstract}

The availability and reliability of mobile positioning algorithms depend on both the quality of measurements and the environmental characteristics. The positioning systems based on global navigation satellite systems (GNSS), for example, have typically a few meters accuracy but are unavailable in signal denied conditions and unreliable in multipath environments. Other radio network based positioning algorithms have the same drawbacks. This thesis considers a couple of cases where these drawbacks can be mitigated by model-based sensor fusion techniques.

The received signal strength (RSS) is commonly used in cellular radio networks for positioning due to its high availability, but its reliability depends heavily on the environment. We have studied how the directional dependence in the antenna gain in the base stations can be compensated for. We propose a semiempirical model for RSS measurements, composed of an empirical log-distance model of the RSS decay rate, and a deterministic antenna gain model that accounts for non-uniform base station antenna radiation. Evaluations and comparisons presented in this study demonstrate an improvement in estimation performance of the joint model compared to the propagation model alone.

Inertial navigation systems (INS) rely on integrating inertial sensor measurements. INS as a standalone system is known to have a cubic drift in the position error, and it needs supporting sensor information, for instance position fixes from GNSS whenever available. For pedestrians, special tricks such as parametric gait models and step detections can be used to limit the drift. In general, the more accurate gait parameters, the better position estimation accuracy. An improved pedestrian dead reckoning (PDR) algorithm is developed that learns gait parameters in time intervals when direct position measurements (such as GNSS positions) are available. We present a multi-rate filtering solution that leads to improved estimates of both gait parameters and position. To further extend the algorithm to more realistic scenarios, a joint classifier of the user's motion and the device's carrying mode is developed. Classification of motion mode (walking, running, standing still) and device mode (hand-held, in pocket, in backpack) provides information that can assist in the gait learning process and hence improve the position estimation. The algorithms are applied to collected data and promising results are reported. Furthermore, one of the most extensive datasets for personal navigation systems using both rigid body motion trackers and smartphones is presented, and this dataset has also been made publicly available. 



\section{Populärvetenskaplig sammanfattning}

Våra smarta mobiler har sofistikerade mätningar och algoritmer för att beräkna användarens position. Utomhus används främst satellitnavigering (GPS), som har en hög noggrannhet men lider av problem med tillgänglighet när man inte har fri sikt till satelliterna (t.ex. inomhus) och problem med tillförlitligheten i komplexa miljöer som stadskärnor, där satellit-signalerna studsar runt innan de når till mottagaren. För att gardera sig mot dessa utmaningar, har våra smarta telefoner algoritmer som använder radiosignaler signaler från våra radionät. För det första kan man utnyttja privata WiFi-nätverk som telefonen hittar och jämföra med en karta där dessa finns utsatta. För det andra används signalstyrkan från våra mobilnät för att avgöra vår position. Detta är två exempel på hur modeller av radiosignaler används för att beräkna position för mobila användare. Denna avhandling behandlar ett par andra fall för att få bättre skattning av position, också ämnade för mobila användare.

Det första fallet är att utnyttja att utsänd effekt från mobilmaster har ett riktningsberoende pga. antennernas utformning. Detta antennmönster är känt av operatören, men inte för mobila användare. Vi studerar hur man kan skatta detta antennmönster och kombinerar detta med en enkel utbredningsmodell för att förklara hur signalstyrkan beror på positionen. Denna modell kan sedan användas 'baklänges' för att räkna ut positionen från uppmätta signalstyrkor från olika basstationer.

Våra smarta telefoner innehåller tröghetssensorer (accelerometer och gyroskop) samt elektronisk kompass (magnetometer), och dessa kan användas för att ge oss en grov modell av hur användaren rör sig, tex genom att använda modeller för gångstil och stegdetektion. Dessa modeller innehåller en mängd parametrar för att beskriva vår gångstil, som dessutom varierar med promenadhastighet eller om vi börjar springa. Signalerna från tröghetssensorerna beror också på var vi har telefonen (i handen, ficka, väska osv.). Ju bättre modell, desto bättre uppfattning av hur vi rör oss. Vi presenterar en algoritm som lär sig dessa gångstils-beroende parametrar under tidsintervall när det finns stödsystem som ger position, tex GPS, och sedan använder denna parametriska modell 'baklänges' för att förbättra rörelsemodellen.

Ett av de mer praktiska bidragen är genomförandet en omfattande datainsamling där av en mängd olika försökspersoner fick röra sig längs en förutbestämd väg med varierande hastigheter i olika faser, samt med en mängd telefoner i olika positioner. För att få ytterligare referensmätningar av gångstil, användes en kroppsdräkt med tröghetssensorer på varje kroppsdel. Detta dataset har gjorts allmänt tillgängligt, och beskrivs utförligt i avhandlingen. Slutligen presenteras lovande resultat från en klassificerare som utifrån telefonens mätningar kan lista ut hur telefonen bärs och vilken promenadstil användare har för tillfället. 



\section{Acknowledgments}

I would like to take this opportunity to express my gratitude to my supervisor, Prof. Fredrik Gustafsson. Your endless, inspiring ideas were the key to the problems that I have encountered, throughout my career as a PhD student. I am deeply grateful for your valuable reflections that facilitated my academic development. I received nothing but complete support and heart-warming words from you during this time.

I would like to thank Prof. Svante Gunnarsson and Prof. Fredrik Gustafsson for giving me the opportunity to join the Automatic Control Group that I am honored to be part of. Prof. Svante Gunnarsson, many thanks for providing us an extraordinary work environment. Ninna Stensgård, I understand that I bothered you a lot with resident-permit related issues, which apparently, happens to me every single year! Thanks a lot for all your help.

I was privileged enough to have great co-supervisors, Dr. Gustaf Hendeby, Dr. Carsten Fritsche, and Dr. Fredrik Gunnarsson. Gustaf, a special thanks for the many hours that you devoted to the questions, concerns, stressful situations and basically everything that I brought to you. Thank you for having a superpower of being unbelievably available all the time, even at nights and weekends. Carsten, thank you for your help with final polishing of the papers. You have an eagle eye for finding subtle mistakes.

I would like to thank Dr. Gustaf Hendeby, Prof. Fredrik Gustafsson and Dr. Carsten Fritsche for your all valuable inputs and proofreading that improved this thesis and its structure. I would also like to thank Prof. Fredrik Gustafsson for helping me with the Swedish summary. As for the proofreading gang, Kamiar Radnosrati, Nader Daneshfar, Lic. Yuxin Zhao and Erik Hedberg, it goes without saying how grateful I am for your comments and time. Dr. Gustaf Hendeby and Dr. Henrik Tidefelt, a big thanks for developing such a comfortable $\mathrm{IAT}_{\mathrm{E}} \mathrm{X}$ template. The writing would not have been this smooth without it.

I would like to gratefully acknowledge the European Union FP7 Marie Curie training program on Tracking in Complex Sensor Systems (TRAX) for the financial support from grant number 607400 .

I am certain that everyone who works in Automatic Control agrees on what an impeccable environment we have. This would not be possible without great colleagues. Thank you all for making such a friendly and amazing atmosphere at work. Angela Fontan, I am happy that you joined the group. You are a good friend and I enjoy spending time with you, we should do it more often. Christian Andersson Naesseth, my former office-mate and new neighbor, thanks for all your tips and help that made everything smoother in the beginning. Martin Lindfors, thanks for helping me with the control labs when I had just started here and most of the assistant manuals were only in Swedish. Finally, thanks to Lic. Yuxin Zhao and Gustav Lindmark, for all of the course-related collaborations and discussions.

Living abroad would be impossible without having close friends and I am honored to have some awesome ones; Mona, Orod, Nader, Afsaneh and Saeed, even though we are not close to each other anymore (distance-wise! And not 
you Nader!), I am always looking forward to weekends, Easter times or other occasions that we get together. Our amazing trips, beer-time over Skype and its consequences that we usually realised the next morning, are all unforgettable. Nader, you are so special! I have never seen such a selfless and caring person like you. Thanks for cheering me up with your perfect blueberry cheesecakes and your Saturday breakfasts with infinite variety of jams and freshly-baked bread. I also would like to thank my friends Sarah and Mohammad for your support, understanding and for sharing your experiences with me whenever I got nervous.

Mom, Dad and Pedi, you know how much I love you and how much I am proud of you. Mom, Dad, without your support and your endless love I would never be able to pursue my dreams to this stage. Thank you so much for dedicating all your life to giving us everything and making us happy. Pedi, life cannot get any better when you are around. You are the best brother ever. PLEASE get here soon!

And of course, I left the toughest one for last. Kamiar, where should I put you! You were first mentioned as one of my colleagues that I am utterly grateful to have. You then jumped few paragraphs down, when I talked about the amazing friends I have, which obviously, you are one of them! And here we are again, when I am talking about my family and guess what, you are part of that too! Can you stop moving around my acknowledgment please?! Just like this acknowledgment, you have also left your footprints all over my heart.

Linköping, September 2017 Parinaz kasebzadeh 


\section{Contents}

Notation

1 Introduction 1

1.1 Motivation and Background . . . . . . . . . . . 2

1.1.1 Antenna gain parameter estimation . . . . . . . . 2

1.1.2 Pedestrian dead reckoning positioning . . . . . . . . 3

1.1.3 Motion and device mode classification . . . . . . . . . . 4

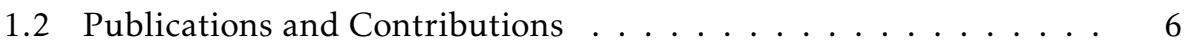

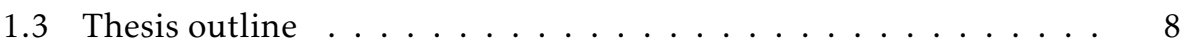

\section{Background}

2 Estimation Prerequisites $\quad 11$

2.1 Parameter Estimation . . . . . . . . . . . . . . . . . . 11

2.1.1 General Definition . . . . . . . . . . . . 12

2.1.2 Maximum Likelihood Estimator . . . . . . . . . . . . . . 12

2.1.3 Least Squares Estimator . . . . . . . . . . . . . . . . 13

2.1.4 Gaussian Noise Scenarios . . . . . . . . . . . . . . . . 14

2.2 State-Space Estimation . . . . . . . . . . . . . . . 14

2.2.1 Stochastic State Space Models . . . . . . . . . . . . . . 14

2.2.2 State Estimation . . . . . . . . . . . . . . . . . . 17

2.2 .3 Kalman Filter . . . . . . . . . . . . . . 19

2.2.4 Extended Kalman Filter . . . . . . . . . . . . 21

3 Pedestrian Dead Reckoning Positioning 23

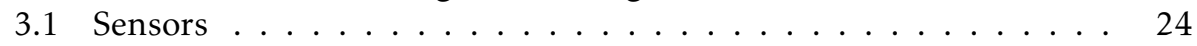

3.2 Sensor Fusion $\ldots \ldots \ldots \ldots \ldots$

3.3 Model Framework . . . . . . . . . . . . . . . . . . . . . . 27

3.4 Supporting measurements . . . . . . . . . . . . . 29

3.4 .1 GPS outdoor . . . . . . . . . . . . . . . . . . 29

3.4 .2 RSS Outdoor . . . . . . . . . . . . . . . . . . . . . . 29

3.4.3 RSS Fingerprint Maps Indoor . . . . . . . . . . 30 
3.4.4 Proximity Sensors . . . . . . . . . . . . . 31

\section{Applications}

4 Antenna Gain Parameter Estimation 35

4.1 Rss Measurement Model . . . . . . . . . . . . . . . . . 35

4.1 .1 Path Loss Model . . . . . . . . . . . . . . . . . . . . . . . . . . . . 36

4.1.2 Antenna Gain Model . . . . . . . . . . . . . . . 37

4.2 Joint Path Loss and Antenna Parameter Estimation . . . . . . . . . 37

4.3 Simulated Data Experiments . . . . . . . . . . . . . . . 40

4.4 Real Data Experiments . . . . . . . . . . . . . 42

5 Gait parameter Estimation Learning 45

5.1 Pedestrian Odometry . . . . . . . . . . . . . . . . 46

5.1.1 Extended Pedestrian Odometric Model . . . . . . . . . . . 46

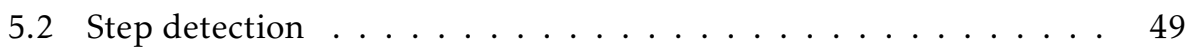

5.2 .1 Step Detection Algorithm . . . . . . . . . . . . 50

5.2.2 Step Length Estimation . . . . . . . . . . . . . . . . 52

5.3 Filter Bank . . . . . . . . . . . . . . . . . 54

5.3.1 Offline Kalman Filter Bank . . . . . . . . . . . . . . . . . 54

5.3.2 Online Kalman Filter Bank . . . . . . . . . . . . . . . 57

5.4 Dead reckoning in different environments . . . . . . . . . . 60

5.4.1 Simulated Environment .................. 60

5.4.2 Real Environment ................663

6 Motion and Device Mode Classification $\quad 73$

6.1 Experiment Setup . . . . . . . . . . . . . . . 73

6.1 .1 Sensors ...................... 74

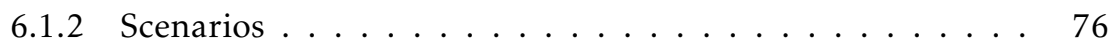

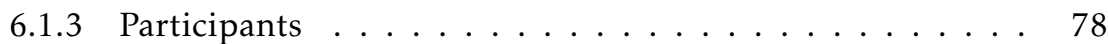

6.2 Available Data . . . . . . . . . . . . . . 78

6.2 .1 Collected Data . . . . . . . . . . . . . . . . 78

6.2 .2 Ground Truth . . . . . . . . . . . . . . . . 79

6.2 .3 Acquire Data . . . . . . . . . . . . . . . . 81

6.3 Data Analysis . . . . . . . . . . . . . . . 82

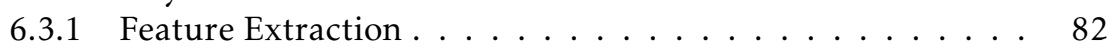

6.3.2 Classification ................. 87

\section{Conclusion and Future works}

7 Concluding Remarks $\quad 91$

7.1 Antenna gait parameters estimation . . . . . . . . . . . . . 92

7.2 Pedestrian dead reckoning positioning . . . . . . . . . . . . . 92

7.3 Motion and Device Mode Classification . . . . . . . . . . . . . 92

7.4 Future Work . . . . . . . . . . . . . . . . . 93 
Bibliography 

Notation

Mathematical Style

\begin{tabular}{cl}
\hline Notation & Meaning \\
\hline$z$ & Scalar parameter or variable \\
$\mathbf{Z}$ & Parameter or variable vector \\
$\mathbf{Z}$ & Parameter or variable matrix \\
\hline
\end{tabular}

Symbols AND OPERATIONS

\begin{tabular}{cl}
\hline Notation & Meaning \\
\hline $\mathbf{x}_{k}$ & State vector at time $k$ \\
$\mathbf{x}_{1: m}$ & Set of states from time 1 to $m$ \\
$\mathbf{u}_{k}$ & Known input vector at time $k$ \\
$\mathbf{y}_{k}$ & Measurements at time $k$ \\
$\mathbf{y}_{1: m}$ & Set of measurements from time 1 to $m$ \\
$\mathbf{f}_{k}(\cdot)$ & State update equation at time $k$ \\
$\mathbf{h}_{k}(\cdot)$ & Measurement equation at time $k$ \\
$\hat{\mathbf{x}}_{k \mid k}$ & State estimate at time $k$ given measurements up to and \\
& including time $k$ \\
$\mathbf{P}_{k \mid k}$ & State covariance at time $k$ given measurements up to \\
$\hat{\boldsymbol{\theta}}$ & and including time $k$ \\
$\mathcal{N}(\mu, \Sigma)$ & Parameter estimate \\
$\arg \max$ & Gaussian distribution with mean $\mu$ and covariance $\Sigma$ \\
$\arg \min$ & Maximizing argument \\
$|\cdot|$ & Euclidean norm of a vector \\
$\|\cdot\|$ & L norm \\
$p(a \mid b)$ & Conditional PDF of stochastic variable \\
$\operatorname{Cov}(\cdot)$ & Covariance \\
$\mathbf{E}(\cdot)$ & Expected value \\
$\mathbb{R}$ & Set of real numbers
\end{tabular}




\begin{tabular}{cl} 
AbBREVIATION & \\
\hline Abbreviation & Meaning \\
\hline BS & Base station \\
CT & Coordinated turn \\
CV & Constant velocity \\
EKF & Extended Kalman filter \\
GNSS & Global Navigation Satellite System \\
IPS & Indoor positioning system \\
IMU & Inertial measurement unit \\
INS & Inertial navigation system \\
IMM & Interacting multiple model \\
KF & Kalman filter \\
LSE & Least squares estimator \\
LTE & Long-term evolution \\
LOS & Line of sight \\
MLE & Maximum likelihood estimator \\
MEMS & Micro-machined electromechanical systems \\
MS & Mobile station \\
PDR & Pedestrian dead reckoning \\
PNS & Pedestrian Navigation System \\
RSS & Received signal strength \\
SSM & State-space model \\
TOA & Time of arrival \\
WLAN & Wireless local area network \\
3GPP & Third generation partnership project \\
\hline &
\end{tabular}




\section{1}

\section{Introduction}

In this thesis, we investigate parameter estimation problems for mobile positioning applications based on measurements provided by common smartphones. The measurements include received signal strength (RSS) which depends on the distance to the radio transmitter but also on the environment and other uncertainties. There are well established but approximate parametric models that describe how RSS depends on distance and direction to the transmitter, and if these parameters were known one could trilaterate the position of the mobile user given a couple of RSS values from different transmitters.

Conversely, the smartphones also contain inertial sensors and an electronic compass that can be used to detect user movement and estimate the speed and direction of the movement. Together, these can be seen as inputs to a motion model for the mobile user, a so called gait model. Again, there are parameters in this gait model that need to be adopted to each user and each use case.

The gait model and the RSS model can be processed by a nonlinear filter model, if only the parameters were known. This parameter estimation problem is the focus in this thesis. The proposed RSS model jointly accounts for RSS decay rate and the base station antenna characteristics. The gait model takes advantage of supporting measurements as a solution to cumulative error problem caused by using inertial sensors. A more sophisticated approach is to utilize the user's behavior. Knowing the user's motion behavior allows for adopting the gait model parameters accordingly. It is studied how this can be learned from measurements through classification algorithms.

Part I of this thesis provides the basis for the work in Part II, in which three applications are presented and further studied. The motivation and background of this work are introduced in Section 1.1. Section 1.2 presents the author's contributions followed by the outline of the thesis given in Section 1.3. 


\subsection{Motivation and Background}

The high pace of development of geographical positioning methods along with tracking mobile users can be traced back to various sources. People and assets tracking, mapping the location of disaster victims, Navigation, and location based security, are all examples of different applications and services which rely on accurate position estimation [14].

The Global Positioning System (GPS), the first of the Global Navigation Satellite System (GNSS), is still the enabler in many applications. Briefly speaking, the GNSS methods are based on signals transmitted from satellites. However, the limiting factors require these methods to be evolved. For instance, in outdoor environments such as street canyons, bad weather conditions resulting in poor signals, and indoor environments where the signal is totally non-accessible, other alternatives must be applied.

Assisting GNSS signals with information from other wireless networks such as the long term evolution (LTE) or IEEE 802.11, which are studied widely in the literature $[64,93,17,68,67]$, is one solution to increase both availability of service and the accuracy of estimation. On the other hand, in indoor environments, relying on available wireless local area network (WLAN) infrastructure for position estimation purposes has been investigated for long time $[46,24,16,19,91,53,90]$.

In this thesis, we use measurements collected by sensors and receivers available in most recent smartphones. These measurements are used in three different applications as follows. The motivation and background theory for first two applications are previously published by the author in [47, ( 2015 IEEE], [48, (02016 IEEE], and the third application will be published in [49, C2017 IEEE].

\subsubsection{Antenna gain parameter estimation}

Cellular radio network positioning can be seen as an alternative to the GNSS systems mentioned above, when they are unavailable. Emergency call positioning is an example use case. Furthermore, from a radio network management perspective, the positioned radio measurement enables an operator to identify where issues such as poor coverage or excessive interference are located.

The positioning solutions can be characterized as network-centric and mobilecentric. In the former, a network entity estimates the position of a terminal, possibly based on measurements reported by the terminal. In the latter, the terminal is provided with assistance data to enable it to estimate its position. The position estimate may be based on a measurement snapshot, or a time series of measurements. The measurements are typically related to time of arrival (TOA), RSS and angle of arrival (AOA) of transmitted reference signals, or combinations on them [34].

RSS measurements are typically reported from the terminal to the base station for other reasons than positioning, such as handover from a serving cell to a target cell, radio resource management in general, or to assess the properties of the radio conditions in a cell as part of network management. Therefore, they can be seen as readily available. In a positioning context, RSS measurements are used 
for ranging, fingerprinting and channel modeling [61, 8, 34, 65, 92]. Recently, it has also been shown how RSS measurements together with information about base station antenna properties, can be used to estimate the AOA [31]. In addition, knowledge about the serving sector cell together with information about the sectorized antenna, yields a crude estimate of the AOA for the terminal. The performance of positioning based on such bearing estimates is assessed in [13], and in [34]. The latter also addresses the positioning performance given RSS measurements used for ranging, given a parametric radio propagation model.

These models, however, suffer from lack of considering simultaneous effects of channel and antenna parameters on RSS measurements used for positioning algorithms. The changes of channel and antenna parameters based on the instantaneous propagation condition are neglected. If detailed propagation model calibration is ruled out, the applicability of the RSS measurements for ranging is subject to significant uncertainty. Such uncertainty can be tolerated in the positioning algorithm, but still with fairly inaccurate positioning performance as a result [81].

To improve performance, an alternative is to take advantage of the recent developments of smartphones. By logging accurate GPS positions and RSS measurements, it is possible to retrieve calibration measurements and use them for parameter calibrations. Traditionally, close range measurements have been used to measure the antenna gain in detail, while avoiding significant propagation effects. Moreover, positioned RSS measurements have been used together with the antenna models to determine the corresponding RSS measurements from an isotropic antenna, which in turn has been used to estimate the parameters of the propagation model.

\subsubsection{Pedestrian dead reckoning positioning}

The dead reckoning principle can be applied to a pedestrian navigation system (PNS) in order to locate the mobile user in indoor and/or outdoor environments. These systems are gaining increasing interest as a tool to improve the localization aspects specifically in indoor-based problems. In these cases, either the accuracy of the GPS is degraded significantly or the signal is totally inaccessible, due to blocking line-of-sight (LOS) or strong signal attenuation. The application areas of PNSs are many, where navigation for blind, helping people suffering from Alzheimer's, emergency coordination, assets tracking, rescue, and tracking in big malls are a few examples.

Traditionally, PNSs use micro-electromechanical systems (MEMS) in order to locate the mobile user when GPS signals are blocked. Strap-down inertial navigation system (INS) is one example of PNSs that takes advantage of MEMS sensors for the positioning process. However, these systems are not self-contained navigation systems. The reason comes from the positioning error caused by gyroscope and accelerometer resulting in a rapid drift growth in such systems.

Various systems and algorithms for PNSs have been introduced in the literature. Comparing them reveals that for pedestrian navigation technology, Pedestrian Dead Reckoning (PDR) using inertial measurement units IMUs has attracted 
the most interest as it imposes no extra cost and does not rely on additional infrastructure.

PDR integrates embedded IMUs to detect when the user takes footsteps and how the direction changes between footsteps. The IMUs use gyroscopes to determine the heading, and accelerometers to estimate gait parameters such as the number of steps and step lengths. More details about these systems will be presented in Section 3.1. For estimating the gait parameters, it is important to detect step occurrences and their length. These gait characteristics depend on individual walking patterns and vary between people. Besides, the same person does not have the same gait in all situations. That is, step length is a time-varying process which depends on the speed and frequency of steps. These are the main challenges of using PDR algorithms. More details about these systems will be presented in Section 5.2.

PNSs can be generally classified based on the location of the installed sensors. The most popular classes are waist-mounted $[4,56]$, foot-mounted $[60,11]$ and hand-held $[58,83,85,94]$ types. Besides the large class of IMU-based systems, there are also other approaches that use other sensors such as electronic pedometers [42]. Chapter 6 is dedicated to discussing this issue.

Since body-fixed sensors, introduced above, require extra devices to be produced and mounted, hand-held devices gain more interest thanks to the rapid development of smartphones. One example of using a hand-held device for positioning purposes is presented by [58]. It uses an empirical model which is based on the accelerometer signal for detecting steps and a back-propagation neural network for step length estimation. In [51], a step determination method based on pattern recognition is proposed.

Another class of methods used in this context are methods that use constant, pre-learned gait parameters. As mentioned before, the step length depends on the user's behavioral and physical characteristics. In order to take this relation into account, a few parameters such as weight and height must be calibrated before starting measurement and performing gait parameter estimation. Different studies on this problem propose constant pre-learned parameters in their models. For example, in [55] a linear relation between the measured frequency of steps and a pre-learned constant parameter is proposed for online step length estimation. In [77], the step frequency and variation of the acceleration is taken into account together with a pre-learned constant parameter.

In this thesis, a filtering approach that can learn gait parameters of the PDR algorithm, such as the step detection threshold and step length, is proposed. This approach is based on a multi-rate Kalman filter bank that estimates the gait parameters when position measurements from GPS are available, which improves PDR in time intervals when GPS position estimates are unavailable. Fig. 1.1 is an illustration of a smartphone using IMUs for positioning purposes.

\subsubsection{Motion and device mode classification}

PNS is used in a range of applications, from pure navigation and guidance tools, healthcare assistance systems to infotainment applications, and more generally 


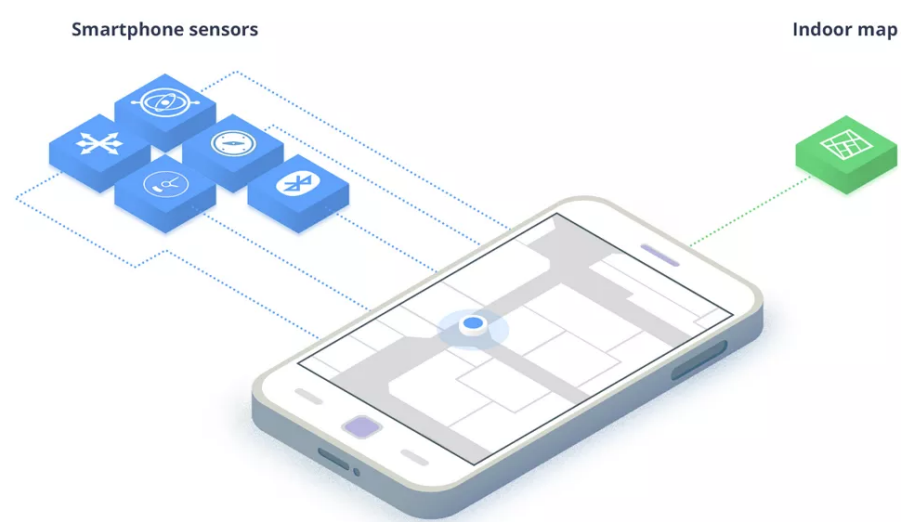

Figure 1.1: Indoor positioning using smartphone. By courtesy of Senion [2].

location based services. The main goal of a PNS is to have an accurate and reliable position estimate, but there are certain metadata that can provide additional information in itself. In this contribution, the problem of classifying the activity mode (standing still, walking, running) and the device mode (handheld in view, handheld in swinging hand, in front/back pocket, and in a backpack) is considered.

The activity mode is a key feature in sports and healthcare applications, where it is logged for its own sake. The activity mode can also select a set of appropriate internal parameters in the PNS, such as step length and step detection thresholds [48]. It can also be an enabler for energy efficient PNS. For instance, in outdoor applications, an energy demanding GPS fix can be obtained first when the user has moved a certain distance, and here it is useful to know if the user is standing still, walking or running. Similar compromises about using additional information sources from infrastructure can be made in indoor PNS. For certain personnel, such as guards and rangers, running may indicate danger and a sudden and unexpected stand still can indicate an accident, and in both cases officers can be automatically alerted.

The device mode is crucial for the design and performance of a PNS. For instance, if it is known that the device is rigidly attached to a foot, special tricks can be used [26, 11, 60]. Most importantly, using the knowledge that the foot is at rest, at least for a short while in each stance, the bias in the accelerometer and gyroscope can be read off directly. These are referred to as zero velocity updates and zero angular rate updates, respectively. The elimination of bias enables the use of dead-reckoning principles to integrate acceleration and angular rate into a precise trajectory.

Other assumptions on the device mode include that the IMU is fixed on the waist rather than the foot $[4,56]$, located in the front pocket [79], carried horizontally in hand [32] or carried in hand not necessarily horizontally [58, 83, 85, 94].

Classification of various motion modes could be one step towards more real- 
istic scenarios in which the smartphone is allowed to switch arbitrarily among different device modes, just as normal users operate their smartphones. Classification of motion and device modes is a less studied area in literature. However, some studies are presented in the following. The classifier introduced in [66] is based on accelerations and magnetic field data recorded with a hand-held unit. Another study dealing with different motion and device modes is performed and reported in [80], where standing still and walking patterns are studied. An extended investigation is to also add the running mode as in [94]. The classification of motion mode is also studied in $[20,21,82,70]$.

The importance of the modes classification for PNS can be summarized as follows. The main design parameters include the step length and the step detection threshold determining when the magnitude of the measured acceleration is deemed to be caused by a step. Both of these depend on the motion mode. Basically, the smaller the step length, the smaller the threshold needs to be. The device mode can simplify the model further. For instance, if the device is hand held flat, the heading corresponding to the projection (rotation) to the horizontal plane (heading) can be computed by just integrating the angular rate around the gravity vector. There are many other similar tricks described in literature. One recent proposal of a multi-mode PDR algorithm can be found in [82], otherwise mode-switching algorithms seem to be rare in literature.

This application is based on an extensive experimental study where different users participated. Several smartphones were carried in different ways, as well as inertial measurements units configured in a body suit. More details about the experimental setup and the methods are given in Chapter 6. Fig. 1.2 shows a subject while collecting data wearing a suit, provided by Xsens, containing the IMU sensors and carrying several smartphones.

\subsection{Publications and Contributions}

The main contributions and publications of the work can be listed as follows

1. The development and analysis of models for jointly antenna and propagation model parameters estimation. The contribution is published in the paper

P. Kasebzadeh, C. Fritsche, E. Özkan, F. Gunnarsson, F. Gustafsson. Joint Antenna and Propagation Model Parameter Estimation using RSS measurements. In Proceedings of the 18th International Conference on Information Fusion, pages 98-103, Washington D. C., USA, July 2015.

This contribution corresponds to the material presented in Chapters 1, 4, and 7. The generic models that form the basis of parameter estimation models used in this application are presented and described in Section 2.1.

2. Development of an extension of a pedestrian dead reckoning model. Some parts of the contribution is published in the paper 


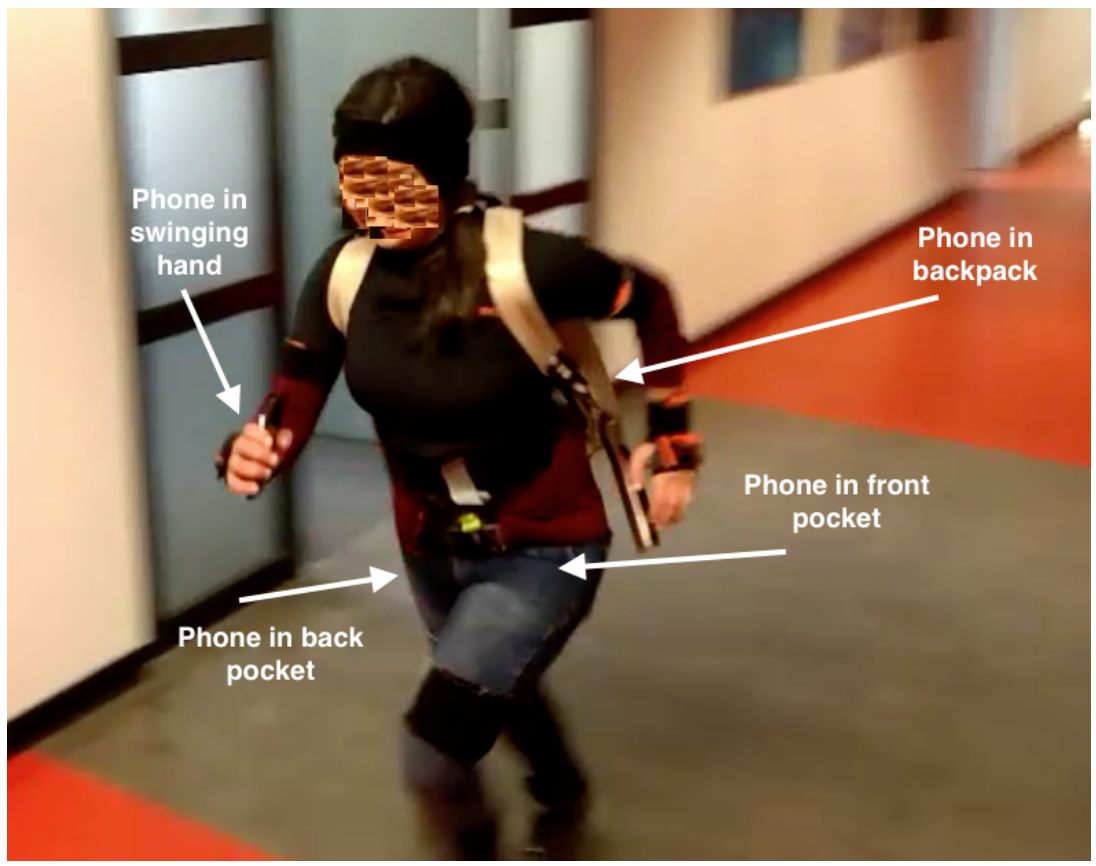

Figure 1.2: Photo from measurement campaign. Activity recognition, the subject is running while carrying four smartphones in swinging hand, in her front and back pocket, and also in her backpack [49, O2017 IEEE].

P. Kasebzadeh, C. Fritsche, G. Hendeby, F. Gunnarsson, F. Gustafsson. Improved Pedestrian Dead Reckoning Positioning With Gait Parameter Learning. In Proceedings of the 19th International Conference on Information Fusion, pages 379-385, Heidelberg, Germany, July 2016.

This contribution corresponds to the material presented in Chapters 1, 5 and 7 . The models that are used for state estimation in this application are presented in Section 2.2. The version presented in this thesis is an extended version of the paper.

3. Classification of human motion activity modes and device modes. The contribution is published in the paper

P. Kasebzadeh, G. Hendeby, C. Fritsche, F. Gunnarsson, F. Gustafsson. IMU Dataset For Motion and Device Mode Classification. In Proceedings of the 8th International Conference on Indoor positioning and indoor navigation, Sapporo, Japan, September 2017.

This contribution corresponds to the material presented in Chapters 1, 6 and 7. 
The content of these three contributions are reused in this thesis by courtesy of IEEE.

\subsection{Thesis outline}

This thesis is divided into two parts and founded upon theories formulated in estimation theory and machine learning. The first part is devoted to the theoretical backgrounds relevant to the applications which are introduced in the second part. These applications are the edited and/or extended versions of published papers listed in Section 1.2.

In Chapter 2, we describe the relevant background information for the rest of this work. Representations of state space models, common dynamics, and measurement models are provided in this chapter. Given a set of measurements, the measurement model is used to infer the desired parameters.

In Chapter 3, we present a general odometry model for PDR and also introduce supporting measurements used to increase accuracy and reliability of the system. Furthermore, detailed measurement model descriptions to relate the observed quantity to the desired state to be estimated are provided. Additionally, we describe all the sensors and receivers that are used as the measurement tools.

Chapter 4 is dedicated to the first contribution in Section 1.2. In this application, a semi-empirical model for RSS measurements is introduced that can be used to predict base station antenna coverage in cellular radio networks. The model is composed of an empirical log-distance model and a deterministic antenna gain model that accounts for possible non-uniform base station antenna radiation. A least squares estimator is proposed to jointly estimate the path loss and antenna gain model parameters. Efficacy of this application is verified in both simulated and experimental fields.

Chapter 5 presents second contribution introduced in Section 1.2. We consider PNSs in devices equipped with inertial sensors and GPS, where we propose an improved PDR algorithm that learns gait parameters in time intervals when GPS is available. A novel filtering approach is proposed that is able to learn internal gait parameters in the PDR algorithm, such as the step length and the step detection threshold. Our approach is based on a multi-rate Kalman filter bank that estimates the gait parameters when position measurements are available, which improves PDR in time intervals when the position is not available.

Chapter 6 is dedicated to the third contribution in Section 1.2. In this chapter, we study the classification of human motion modes (walking, standing still and running) and device modes (hand-held, in pocket, in backpack). This classification is an enabler in PNSs for the purpose of saving energy and design parameter settings and also for its own sake. The main contribution is to publish one of the most extensive datasets for this problem. Moreover, a first study on a joint human motion and device mode classifier is presented.

The work is concluded and a discussion of the future work is presented in Chapter 7. 
Part I

\section{Background}





\section{2}

\section{Estimation Prerequisites}

Estimation is the process in which imprecise and uncertain observations are used for inferring the "best guess" of a quantity of interest. In the context of this work, estimation is the basis for determining parameters or states of a dynamic system. In other words, it is applied to maximize the knowledge about an unknown variable given unreliable observations.

This chapter sets the stage for all subsequent chapters by introducing the key problems considered in this thesis; parameter estimation for nonlinear models and estimation of unknown states of a dynamic system. Section 2.1 defines the generic estimation problems and methods used for solving these problems. In Section 2.2, a general introduction to stochastic state-space models is given. Finally, three different types of state estimation problems are defined, including the filtering problem that is the main idea of this thesis.

\subsection{Parameter Estimation}

Parameter estimation models use the measured data to obtain the best estimate of the deterministic parameters. The term parameter is used to designate a quantity, scalar or vector valued, that is assumed time invariant [9].

A broad variety of parameter estimation techniques are presented by $[50,54]$. This section presents an overview of two well-known methods used for data analysis; maximum likelihood (ML) and least squares (LS) estimation.

In order to estimate the unknown parameters, the estimator uses information from the measurement model, probabilistic characterization of the various random factors such as disturbances, and the evaluation of the variables from the dynamic system. 


\subsubsection{General Definition}

Unknown parameters $\theta$ can be associated with the measured data using a generic measurement model

$$
y_{i}=h\left(\mathbf{u}_{i}, \boldsymbol{\theta}\right)+e_{i} \quad i=1, \ldots, m,
$$

where $\mathbf{u}_{i}$ is the known input vector, $\theta$ is the vector of unknown parameters belonging to the parameter space $\Theta \subset \mathbb{R}^{n}$, and errors $e_{i}$ are unobserved independent random variables with zero means and finite variances. Here, the problem is to infer $\theta$ from noise measurements, $y$. The inference is performed in the statistical framework. In the non-Bayesian approach, which is the scope of this section, no prior knowledge of the unknown parameters are assumed. Thus, no prior distribution is associated with the parameters. The estimated parameters $\hat{\theta}$ are given by

$$
\hat{\theta} \triangleq\left[\begin{array}{c}
\hat{\theta}_{1} \\
\hat{\theta}_{2} \\
\vdots \\
\hat{\theta}_{n}
\end{array}\right] .
$$

Two common alternatives to find $\hat{\theta}$ in (2.2) from measurements (2.1) are maximum likelihood and least squares estimators. Both methods are based on statistical procedures that are explained in more detail in the following. In brief, the least squares method is used to estimate the coefficients in a model by minimizing the sum of squares of the differences between fitted values and observed values regardless of the form of the distribution of the errors. The least squares produces the best linear unbiased estimate of those coefficients, in linear models. However, if the form of the distribution of the errors is known, the maximum likelihood estimator (MLE) can alternatively be used to estimate those coefficients. Although the optimization criterion is different between the two methods, it will be shown that under certain assumptions they are equivalent.

\subsubsection{Maximum Likelihood Estimator}

Maximum likelihood estimation (MLE) is a well-known method for estimating nonrandom parameters that uses given observations to estimate the parameters. Let $\mathbf{y}=\left[y_{1}, \ldots, y_{m}\right]^{T}$ be a random vector of observations with probability density function (PDF) $p\left(y_{i} ; \boldsymbol{\theta}\right)$.

The joint distribution of the measurement, assumed to be independent, identically distributed (i.i.d.), is given by the product of the marginal densities

$$
p\left(y_{1}, \ldots, y_{m} ; \boldsymbol{\theta}\right)=\prod_{i=1}^{m} p\left(y_{i} ; \boldsymbol{\theta}\right) .
$$


The joint density is an $m$-dimensional function of the data $y_{1}, \ldots, y_{m}$ given the parameter vector $\theta$ satisfying

$$
\begin{aligned}
p\left(y_{1}, \ldots, y_{m} ; \boldsymbol{\theta}\right) & \geq 0, \\
\int \ldots \int p\left(y_{1}, \ldots, y_{m} ; \boldsymbol{\theta}\right) \mathrm{d} y_{1} \ldots \mathrm{d} y_{m} & =1 .
\end{aligned}
$$

For a given realization $\mathbf{y}$, the likelihood function of $\mathbf{y}$ is considered as a function of $\theta$, i.e.

$$
\mathrm{L}(\boldsymbol{\theta} \mid \mathbf{y})=\prod_{i=1}^{m} p\left(y_{i} ; \boldsymbol{\theta}\right)=p(\mathbf{y} ; \boldsymbol{\theta}) .
$$

Note that the likelihood function is a function of the parameters $\theta$ and not the data $\mathbf{y}$ and thus is not a proper pdf, i.e. generally

$$
\int \ldots \int \mathrm{L}\left(\boldsymbol{\theta} \mid y_{1}, \ldots, y_{m}\right) \mathrm{d} \theta_{1} \ldots \mathrm{d} \theta_{n} \neq 1 .
$$

The MLE finds the parameter values by maximizing the likelihood function that serves as a measure of the evidence from the data. The MLE of the unknown true parameter $\theta$ is the one that maximizes (2.5) over $\Theta$

$$
\hat{\boldsymbol{\theta}}^{\mathrm{ML}}=\arg \max _{\boldsymbol{\theta} \in \Theta} \mathrm{L}(\boldsymbol{\theta} \mid \mathbf{y})=\arg \max _{\boldsymbol{\theta} \in \Theta} p(\mathbf{y} ; \boldsymbol{\theta}) .
$$

It is often easier to maximize the $\log$-likelihood function $\ln \mathrm{L}(\boldsymbol{\theta} \mid \mathbf{y})$ instead of direct maximization of $\mathrm{L}(\theta \mid \mathbf{y})$. Since $\ln (\cdot)$ is a monotonically increasing function, the value of $\boldsymbol{\theta}$ that maximizes $\ln \mathrm{L}(\boldsymbol{\theta} \mid \mathbf{y})$ also maximizes $\mathrm{L}(\boldsymbol{\theta} \mid \mathbf{y})$.

\subsubsection{Least Squares Estimator}

The least squares estimator (LSE) is another well-known method for estimating nonrandom parameters that uses given measurements. LSE estimates parameters by minimizing the squared discrepancies between the measured data and their expected values.

Let $\mathbf{h}(\mathbf{u}, \boldsymbol{\theta})=\left[h\left(\mathbf{u}_{1}, \boldsymbol{\theta}\right), \ldots, h\left(\mathbf{u}_{m}, \boldsymbol{\theta}\right)\right]^{T}$ and $\mathbf{e}=\left[e_{1}, \ldots, e_{m}\right]^{T}$ be points in $\mathbb{R}^{n}$. The generic model defined in (2.1) specifies a surface $M_{\Theta}=\{\mathbf{h}(\mathbf{u}, \theta): \theta \in \Theta\}$ in $\mathbb{R}^{n}$. The LSE estimate $\hat{\theta}^{\mathrm{LS}}$ of the unknown parameters is the one that minimizes the distance from $y$ to $M_{\Theta}$. Thus, the LSE of $\theta$, considering (2.1), can be expressed as

$$
\hat{\boldsymbol{\theta}}^{\mathrm{LS}}=\arg \min _{\boldsymbol{\theta} \in \Theta}\left\{\sum_{i=1}^{m}\left|y_{i}-h\left(\mathbf{u}_{i}, \boldsymbol{\theta}\right)\right|^{2}\right\},
$$

where $|\cdot|$ denotes the Euclidean norm. In the special case where (2.1) is linear in the parameters $\theta$, i.e.

$$
\mathbf{h}(\mathbf{u}, \boldsymbol{\theta})=\mathbf{H}(\mathbf{u}) \boldsymbol{\theta}
$$


there exists a closed form solution to (2.8) given by

$$
\hat{\boldsymbol{\theta}}^{\mathrm{LS}}=\left(\mathbf{H}(\mathbf{u})^{T} \mathbf{H}(\mathbf{u})\right)^{-1} \mathbf{H}(\mathbf{u})^{T} \mathbf{y} .
$$

However, numerical iterative algorithms are usually required whenever the model is nonlinear.

\subsubsection{Gaussian Noise Scenarios}

The criterion in (2.8) does not enforce any assumption on the measurement error. In cases where measurement errors, $e_{i}$, are (i.i.d) zero-mean Gaussian random variables

$$
e_{i} \sim \mathcal{N}\left(0, \sigma^{2}\right),
$$

then the LSE (2.8) estimator is identical to the MLE, where for $i=1, \ldots, m$

$$
y_{i} \sim \mathcal{N}\left(h\left(\mathbf{u}_{i}, \boldsymbol{\theta}\right), \sigma^{2}\right) .
$$

The likelihood function of $\theta$ is then

$$
\begin{aligned}
L(\boldsymbol{\theta} \mid \mathbf{y}) & =p(y ; \boldsymbol{\theta})=p\left(y_{1}, \ldots, y_{m} ; \boldsymbol{\theta}\right) \\
& =\prod_{i=1}^{m} \mathcal{N}\left(y_{i} ; h\left(\mathbf{u}_{i}, \boldsymbol{\theta}\right), \sigma^{2}\right)=\left(\frac{1}{\sqrt{2 \pi \sigma^{2}}}\right)^{m} e^{-\frac{1}{2 \sigma^{2}} \sum_{i=1}^{m}\left[y_{i}-h\left(\mathbf{u}_{i}, \boldsymbol{\theta}\right)\right]^{2}},
\end{aligned}
$$

and the minimization (2.8) is equivalent to the maximization of (2.13), $\hat{\theta}^{\mathrm{LS}}=$ $\hat{\theta}^{\mathrm{ML}}$.

\subsection{State-Space Estimation}

This section begins with a brief introduction to stochastic state-space models. Prediction, filtering, and smoothing of the states are treated separately and explained in Section 2.2.2. The exact and optimal solution to state estimation problems under linearity and known Gaussian noise statistics assumptions is given by the Kalman filter (KF) as explained in Section 2.2.3. Section 2.2.4 relaxes the linearity assumption and provides algorithms for nonlinear state-space models.

\subsubsection{Stochastic State Space Models}

State-space models (SSMs) relate the observed measurements to the latent state variable. The latent variables are not observed, but are reconstructible from the measured data. In the SSM framework, the measurement equation and system dynamics are modeled separately, where the latter predicts how the states of the system evolve over time.

The system dynamics are often continuous in time while observations are taken at discrete time instants. In this work, the system dynamics are restricted 


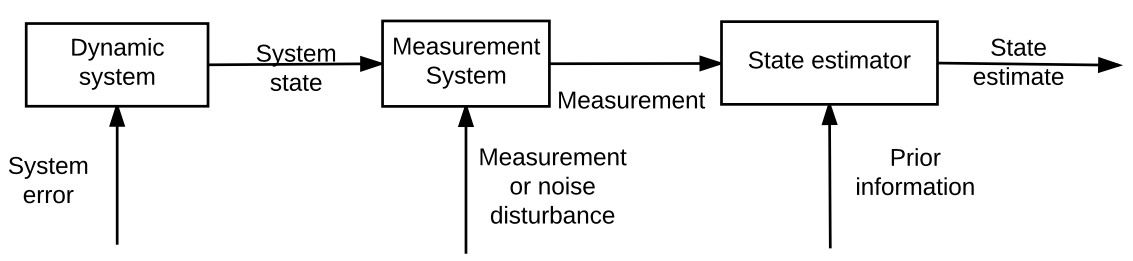

Figure 2.1: Block diagram of the state estimation problem [9].

to discrete time descriptions. These are obtained by discretization of the continuous time dynamics where differential equations are replaced with difference equations.

To better model the characteristics of real systems, a method for predicting behaviors with some kind of randomness is required. Thus, a stochastic SSM is used to model a signal by introducing stochastic variables into the SSM [7]. The randomness can be modeled in both continuous and discrete times. However, stochastic variables in discrete time are studied in this work. An introduction to stochastic SSM is given in $[7,59]$.

\section{General Descriptions}

A generic form of stochastic SSM equations for the $k$-th time index can be given as

$$
\begin{aligned}
\mathbf{x}_{k+1} & =\mathbf{f}_{k}\left(\mathbf{x}_{k}, \mathbf{w}_{k}\right), \\
\mathbf{y}_{k} & =\mathbf{h}_{k}\left(\mathbf{x}_{k}, \mathbf{e}_{k}\right),
\end{aligned}
$$

where $\mathbf{x}_{k} \in \mathbb{R}^{n}$ is the current state vector, and $\mathbf{w}_{k} \in \mathbb{R}^{n_{\mathrm{w}}}$ is the unmeasured disturbance input to the system. $\mathbf{x}_{k+1}$ is the upcoming state, and $\mathbf{e}_{k} \in \mathbb{R}^{n_{e}}$ is the measurement noise and $\mathbf{y}_{k} \in \mathbb{R}^{m}$ contains measurements. The state difference (2.14a) uses a function $\mathbf{f}_{k}(\cdot)$, to account for the relation between the current state and the process noise with the upcoming state. The measurement equation $(2.14 \mathrm{~b})$ utilizes a function $\mathbf{h}_{k}(\cdot)$ to connect the current state and the measurement noise $\mathbf{e}_{k}$ to $\mathbf{y}_{k}$.

The initial state $\mathbf{x}_{0}$, the noise signals $\mathbf{w}_{k}$ and $\mathbf{e}_{k}$ are the stochastic variables of the SSM. The probabilistic state-space model and the stochastic variables $\mathbf{w}_{k}$, and $\mathbf{e}_{k}$ are defined as

$$
\begin{aligned}
\mathbf{x}_{k} & \sim p\left(\mathbf{x}_{k} \mid \mathbf{x}_{k-1}\right), \\
\mathbf{y}_{k} & \sim p\left(\mathbf{y}_{k} \mid \mathbf{x}_{k}\right), \\
\mathbf{w}_{k} & \sim p\left(\mathbf{w}_{k}\right), \\
\mathbf{e}_{k} & \sim p\left(\mathbf{e}_{k}\right), \\
\mathbf{x}_{0} & \sim p\left(\mathbf{x}_{0}\right) .
\end{aligned}
$$


Generally, marginal probability densities are used as in (2.14) with complementary information about correlation between $\mathbf{x}_{0}, \mathbf{w}_{k}$, and $\mathbf{e}_{k}$. The notation in $(2.14 \mathrm{~g})$ means that $\mathbf{x}_{0}$, for instance, has a distribution with density $p\left(\mathbf{x}_{0}\right)$.

Some assumptions are made for the problems considered in this work. First, time index starts at $k=0$ and the first measurement is taken at the next time, $k=1$. At each time instance $k$, both functions $\mathbf{f}_{k}(\cdot)$ and $\mathbf{h}_{k}(\cdot)$ are known. The noise, $\mathbf{w}_{k}$ and $\mathbf{e}_{k}$, characteristics as well as the distribution of $\mathbf{x}_{0}$ are also assumed to be known. For simplicity, the noises are assumed to be zero mean Gaussian independent random variables. Moreover, the initial state $\mathbf{x}_{0}$ is assumed to be independent to the measurement and process noises.

As stated earlier, (2.14) corresponds to a very generic SSM, spanning a broad range of features. For example, there is no restriction on functions $\mathbf{f}_{k}(\cdot)$ and $\mathbf{h}_{k}(\cdot)$ in terms of time-varying or time-invariant characteristics. Furthermore, nonlinearity in both functions can be of any kind. Stochastic variables can also follow any arbitrary distribution. However, a common special case of (2.14) is the stochastic SSM with nonlinear states in the dynamic model with a white Gaussian noise entering the model additively. It is called the additive white Gaussian noise state-space model

$$
\begin{aligned}
\mathbf{x}_{k+1} & =\mathbf{f}_{k}\left(\mathbf{x}_{k}\right)+\mathbf{w}_{k}, \\
\mathbf{y}_{k} & =\mathbf{h}_{k}\left(\mathbf{x}_{k}\right)+\mathbf{e}_{k},
\end{aligned}
$$

where transition function, $\mathbf{f}_{k}(\cdot)$, and measurement function, $\mathbf{h}_{k}(\cdot)$, are both arbitrary nonlinear functions. The dynamic and the measurement models of the system are represented by their corresponding PDFs as $p\left(\mathbf{x}_{k+1} \mid \mathbf{x}_{k}\right)$ and $p\left(\mathbf{y}_{k} \mid \mathbf{x}_{k}\right)$, respectively. The initial state is defined as

$$
\mathbf{x}_{0} \sim \mathcal{N}\left(\overline{\mathbf{x}}_{0}, \mathbf{P}_{0}\right),
$$

and the white Gaussian noise $\mathbf{w}_{k}$ and $\mathbf{e}_{k}$ are independent and with the following distributions

$$
\begin{aligned}
\mathbf{w}_{k} & \sim \mathcal{N}\left(0, \mathbf{Q}_{k}\right), \\
\mathbf{e}_{k} & \sim \mathcal{N}\left(0, \mathbf{R}_{k}\right) .
\end{aligned}
$$

The presented approximate filtering techniques in Section 2.2.4 employ (2.15) with a Gaussian noises assumption. In cases where the dimension of the process noise, $n_{\mathrm{w}}$ is smaller than the dimension of the states, $n$, the $\mathbf{w}_{k}$ is pre-multiplied by a matrix to match the dimensions. Although a similar matrix can be defined and applied on measurement equation, it rarely happens that $n_{e}<m$. Since the obtained measurements are all assumed to be uncertain and noise corrupted, the measurement noise covariance matrix is positive definite. This implies that the measurement noise can be fully characterized by $p\left(\mathbf{e}_{k}\right)$ and no further modifications are required.

\section{Linear model}

Imposing the linearity assumption on the dynamic and measurement models simplifies the computations considerably. The well-known linear Gaussian state- 
space model is obtained by assuming the functions $\mathbf{f}_{k}(x)$ and $\mathbf{h}_{k}(x)$ to be linear as

$$
\begin{aligned}
\mathbf{x}_{k+1} & =\mathbf{F}_{k} \mathbf{x}_{k}+\mathbf{G}_{k} \mathbf{w}_{k}, \\
\mathbf{y}_{k} & =\mathbf{H}_{k} \mathbf{x}_{k}+\mathbf{e}_{k},
\end{aligned}
$$

where $\mathbf{F}_{k}$ is a transition matrix and $\mathbf{H}_{k}$ is a measurement matrix. These two matrices are assumed to be independent of the state $\mathbf{x}_{k}$.

The inference in the linear model is significantly simplified. The reason is that a linear transformation of the Gaussian distributed initial state, does not change the posterior distribution. That is, all subsequent predictions and states will also be Gaussian distributed.

The optimal unbiased state estimation algorithm, utilizes (2.18) with arbitrary noise distributions and known first two moments to find the estimated states with the lowest mean squared error. The well-known Kalman Filter (KF) introduced in Section 2.2.3 gives the optimal state estimates for linear systems with Gaussian noise.

In cases of nonlinear state-space models, approximate filtering algorithms treat nonlinearities in different ways. Markov Chain Monte Carlo approaches directly estimate the distribution of the states which undergo a nonlinear transformation. Another method is to first derive the approximate linearized equations and then treat them as a linear state-space model. The Extended Kalman Filter (EKF) uses this approach and is introduced in Section 2.2.4.

\subsubsection{State Estimation}

Given a set of measurements $\mathbf{Y}_{1: \ell}=\left[\mathbf{y}_{1}, \ldots, \mathbf{y}_{\ell}\right]$, the estimation problem casts in to the one that infers the state estimates $\hat{\mathbf{X}}_{1: \ell}=\left[\hat{\mathbf{x}}_{1}, \ldots, \hat{\mathbf{x}}_{\ell}\right]$ of the true states $\mathbf{X}_{1: \ell}=\left[\mathbf{x}_{1}, \ldots, \mathbf{x}_{\ell}\right]$.the stochastic difference equation model (2.18) is used for state estimation.

In the Bayesian framework, states are interpreted as random variables with a certain distribution. Given the prior knowledge of the stochastic processes $\mathbf{w}_{k}$, and $\mathbf{e}_{k}$, the objective of the Bayesian state estimation problem is to find the conditional posterior density $p\left(\mathbf{x}_{k} \mid \mathbf{Y}_{1: \ell}\right)$.

The posterior estimation, given all measurements up to $\ell$, gradually becomes computationally intractable when $\ell$ gets large. To tackle this problem, Bayesian state estimation is solved under an additional assumptions to deal with this issue. That is, the state $\mathbf{x}_{k}$, follows a Markov process

$$
p\left(\mathbf{x}_{k} \mid \mathbf{x}_{0}, \ldots, \mathbf{x}_{k-1}\right)=p\left(\mathbf{x}_{k} \mid \mathbf{x}_{k-1}\right) .
$$

Nevertheless, the discussion of practical algorithms is postponed to the next section and here, we continue to describe the concepts behind Bayesian the state estimation problem. Generally, there are three marginal distributions that can be considered as a solution to state estimation problems; filtering, prediction and smoothing. These different distribution are distinguished by the temporal relation between the time, $k$, at which states are estimated and the available measure- 


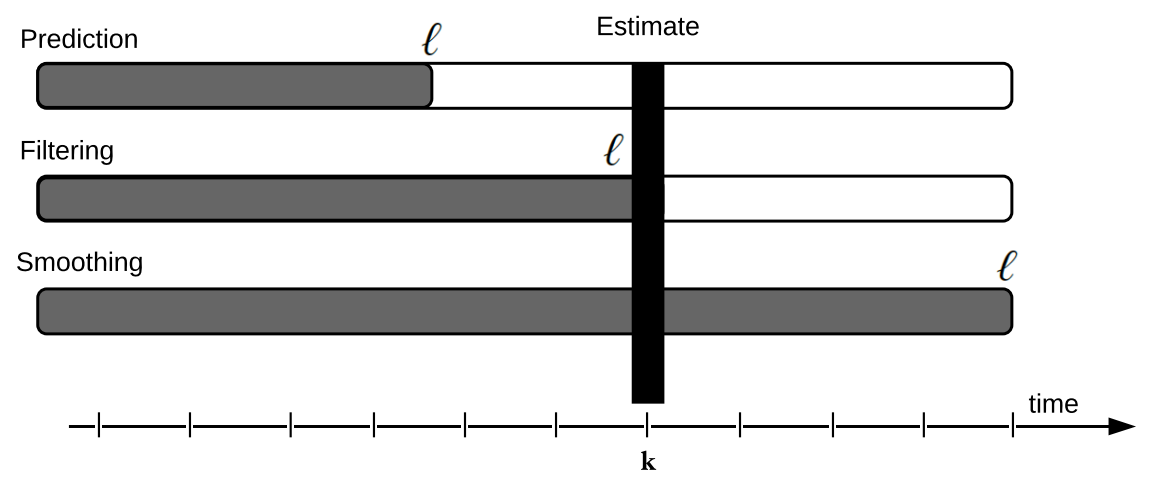

Figure 2.2: Illustration of different estimation problems. the state of interest is indicated with black bar at time $k$. The available measurements up to time $\ell$ are illustrated by gray color [73].

ments time interval, $\ell$, as illustrated in Fig. 2.2 and more thoroughly discussed in $[9,73]$.

\section{- Prediction}

The prediction phase of the Bayesian filter computes the prediction distributions. The first row in Fig. 2.2 illustrates a prediction scheme, in which $\ell<k$. The first step in the prediction is to obtain the transition density $p\left(\mathbf{x}_{k+n} \mid \mathbf{x}_{k}\right)$ using the system model (2.18a) and the statistical properties of $\mathbf{w}_{k}$.

The predictive posterior density $p\left(\mathbf{x}_{k+n} \mid \mathbf{Y}_{1: \ell}\right)$ of the future state $\mathbf{x}_{k+n}, n$ steps after the current time step $\ell$, given the available measurements, $\mathbf{Y}_{1: \ell}$, are then obtained by

$$
p\left(\mathbf{x}_{k+n} \mid \mathbf{Y}_{1: \ell}\right)=\int p\left(\mathbf{x}_{k+n} \mid \mathbf{x}_{k}\right) p\left(\mathbf{x}_{k} \mid \mathbf{Y}_{1: \ell}\right) \mathrm{d} \mathbf{x}_{k},
$$

where the density $p\left(\mathbf{x}_{k} \mid \mathbf{Y}_{1: \ell}\right)$ at the previous time step is propagated to $n$ steps ahead through the transition density. More details are given in $[9,73]$.

- Filtering

In the filtering problem, $k$ is equal to $\ell$ and the posterior density distribution which is going to be estimated now takes the form $p\left(\mathbf{x}_{k} \mid \mathbf{Y}_{1: k}\right)$. This can be defined as an online sequential problem where the filtering density can be computed quickly by applying the Bayes' rule to the prediction density.

The second row in Fig. 2.2 illustrates the Bayesian filtering problem where $\ell=k$. The gray part represents the available measurement sets which reaches up to and includes time $k$. 


\section{- Smoothing}

Bayesian smoother computes smoothing distributions. The third row in Fig. 2.2 illustrates Bayesian smoothing problem in which $\ell>k$. The smoothing distributions are the marginal distributions of state $\mathbf{x}_{k}$ given certain measurement interval, $\mathbf{Y}_{1: \ell}$.

As mentioned in the beginning of this section, the final goal is to find a point estimate of the states represented by $\hat{\mathbf{X}}_{1: \ell}$ together with some measure of uncertainty assigned to each estimate. One approach is to estimate the full posterior density, $p\left(\mathbf{x}_{k} \mid \mathbf{Y}_{1: k}\right)$, as introduced in the filtering above, and then define a point estimate and the uncertainty indicator from the statistics of the approximated distribution.

The Kalman filtering algorithm applies to linear Gaussian systems and computes the full posterior $\mathcal{N}\left(\mathbf{x}_{k} ; \hat{\mathbf{x}}_{k \mid k}, \mathbf{P}_{k \mid k}\right)$. This allows for extracting an estimate of the state $\hat{\mathbf{x}}_{k \mid k}$ and covariance $\mathbf{P}_{k \mid k}$. The reason is that a linear transformation of a Gaussian distribution is also a Gaussian whose sufficient statistics are the value of interest. However, as mentioned before, nonlinear and/or non-Gaussian models require approximations and cannot be handled by the Kalman filters. In case of nonlinear models, the extended Kalman filters can be applied.

\subsubsection{Kalman Filter}

The Kalman Filter is a very popular algorithm for estimating the state of linear systems. This filter is named after Rudolph E. Kalman, one of the primary developers of its theory who introduced the method in [44]. The major contribution of the proposed method to the field of linear filtering was removing the stationary requirements. At that period, the Wiener filter and frequency domain approaches were the main approach in the electrical engineering field and they require the process to be stationary. Kalman provided recursive formulas suited for time-varying linear filtering problems. More details about the KF history can be found in [43].

The KF is an optimal estimator in the sense that it minimizes the estimated error covariance given that some presumed conditions are met. Furthermore, as mentioned in [5], for linear systems with arbitrary noise, the KF is the unbiased linear minimum variance estimator. In fact, $\mathrm{KF}$ is a linear filter which updates the mean and the covariance of the estimate to minimize the error of the estimated parameters or states.

The KF uses the measurements that are linearly correlated to the state, to provide instantaneous estimate of the dynamic system. Both the measurements and the state of the linear system are perturbed by white Gaussian noise. In situations where the noise is not Gaussian, the KF is still the best linear unbiased estimator while nonlinear estimators may have better performance.

Being the optimal estimator together with being tailored for implementation purposes make KF a celebrated solution. Furthermore, KF is convenient for online real-time processing that further broadens its applicability. Unlike available snapshot solutions where each measurement is processed separately, the KF fuses multiple information to provide a better estimate. Using the system dynamics 
modeled by physical laws of motion, for example, known control inputs, and the possibility of fusing measurements from different types, make the KF a powerful tool in estimation applications.

The states of linear state-space systems described by (2.18) can be estimated by the KF. There are many alternative ways to represent the KF equations as described and derived in [5] and [43]. In this thesis, the KF is presented with alternating time update phase and the measurement updated phase, where the dynamics of the system is handled and the measurements are fused in the estimate, respectively.

The KF prediction step based on the dynamic model given by (2.18) can be expressed as

$$
\begin{aligned}
\hat{\mathbf{x}}_{k \mid k-1} & =\mathbf{F}_{k} \hat{\mathbf{x}}_{k-1 \mid k-1} \\
\mathbf{P}_{k \mid k-1} & =\mathbf{F}_{k} \mathbf{P}_{k-1 \mid k-1} \mathbf{F}_{k}^{T}+\mathbf{G}_{k} \mathbf{Q}_{k} \mathbf{G}_{k}^{T},
\end{aligned}
$$

where $\hat{\mathbf{x}}_{k \mid k-1}$ is the mean and $\mathbf{P}_{k \mid k-1}$ is the covariance matrix of the Gaussian prior. This prediction gives the new prior distribution $\mathcal{N}\left(\hat{\mathbf{x}}_{k \mid k-1}, \mathbf{P}_{k \mid k-1}\right)$ and is computed from the previous Gaussian posterior $\mathcal{N}\left(\hat{\mathbf{x}}_{k-1 \mid k-1}, \mathbf{P}_{k-1 \mid k-1}\right)$. In the measurement update phase the prior will be updated whenever a measurement is available. This process can be shown as

$$
\begin{aligned}
\hat{\mathbf{y}}_{k} & =\mathbf{H}_{k} \hat{\mathbf{x}}_{k \mid k-1}, \\
\varepsilon_{k} & =\mathbf{y}_{k}-\hat{\mathbf{y}}_{k}, \\
\mathbf{S}_{k} & =\mathbf{H}_{k} \mathbf{P}_{k \mid k-1} \mathbf{H}_{k}^{T}+\mathbf{R}_{k}, \\
\mathbf{K}_{k} & =\mathbf{P}_{k \mid k-1} \mathbf{H}_{k}^{T} \mathbf{S}_{k}^{-1}, \\
\hat{\mathbf{x}}_{k \mid k} & =\hat{\mathbf{x}}_{k \mid k-1}+\mathbf{K}_{k} \varepsilon_{k}, \\
\mathbf{P}_{k \mid k} & =\left(\mathbf{I}-\mathbf{K}_{k} \mathbf{H}_{k}\right) \mathbf{P}_{k \mid k-1},
\end{aligned}
$$

where $\varepsilon_{k}$ and $S_{k}$ are called innovation/residual and innovation/residual covariance matrix, respectively. $\varepsilon_{k}$ denotes the difference between the prediction and its observed output, while $S_{k}$ represents the uncertainty of the predicted output. The $\mathbf{K}_{k}$ is the Kalman gain and is a factor of the correction. $\hat{\mathbf{x}}_{k \mid k}$ and $\mathbf{P}_{k \mid k}$ are the mean and the covariance matrix of the Gaussian posterior, respectively. I is an identity matrix. There are many ways to formulate the covariance update $(2.22 \mathrm{f})$ to have a better preserving symmetry and positive definite terms. For instance, $\mathbf{P}_{k \mid k}$ can be represented as sum of two positive definite symmetric matrices which is called Joseph form and is formulated as

$$
\mathbf{P}_{k \mid k}=\left(\mathbf{I}-\mathbf{K}_{k} \mathbf{H}_{k}\right) \mathbf{P}_{k \mid k-1}\left(\mathbf{I}-\mathbf{K}_{k} \mathbf{H}_{k}\right)^{T}+\mathbf{K}_{k} \mathbf{R}_{k} \mathbf{K}_{k}^{T} .
$$

However, it requires more matrix manipulations compared to (2.22f). The KF algorithm starts with a time update and is initialized with

$$
\begin{aligned}
& \hat{\mathbf{x}}_{0 \mid 0}=\mathbf{x}_{0}, \\
& \mathbf{P}_{0 \mid 0}=\mathbf{P}_{0} .
\end{aligned}
$$




\subsubsection{Extended Kalman Filter}

Although the Kalman filter is an optimal solution to the state estimation problems, some limiting assumptions are applied in its derivation; the predicted state and the measurement are jointly Gaussian distributed. The conditional Gaussian distribution rules have been used to obtain the equations for the measurement update. Therefore, KF can be applied only to linear models. Thus, KF is not applicable to many real life problems where either the dynamics or the measurement models, or both, are nonlinear. The reason is that the joint prediction density, $p\left(\mathbf{x}_{k}, \mathbf{y}_{k} \mid \mathbf{Y}_{1: k-1}\right)$, is not Gaussian for nonlinear systems even if the noise sources are Gaussian. In these situations, the problem needs to be treated with other approximate filtering algorithms. EKF in nonlinear models is demonstrated and evaluated in early studies in $[74,78,41]$.

EKF is an extension to the KF with the possibility of handling nonlinear models. It handles the nonlinearities in the models by approximating the nonlinear models with corresponding linear models. That is, the nonlinear equations are first linearized and then the approximate filtering densities are computed.

Linearization is performed using the first order Taylor series expansion on a nominal state $\overline{\mathbf{x}}$. In cases where the whole nominal state sequence $\overline{\mathbf{X}}_{1: k}$ is available, linearization can be performed offline. Then, the standard KF can be used. However, this approach might yield poor performance if the nominal trajectory deviates from the true $\mathbf{X}_{1: k}$. To overcome this issue, the EKF uses the available information found and estimated by the filter as the linearization points, consecutively. In this method, the latest estimate is regarded as the best available information on the state and is used for linearization in the next iteration.

Using the first order Taylor expansion on the nonlinear SSM (2.15) and simplifying the expressions by assuming that the process noise is additive, $(2.15 \mathrm{a})$ and $(2.15 \mathrm{~b})$ can be used in the time and the measurement update phases, respectively. The prediction phase for the EKF algorithm is given as follows

$$
\begin{aligned}
\hat{\mathbf{x}}_{k \mid k-1} & =\mathbf{f}\left(\hat{\mathbf{x}}_{k-1 \mid k-1}\right), \\
\mathbf{P}_{k \mid k-1} & =\mathbf{f}^{\prime}\left(\hat{\mathbf{x}}_{k-1 \mid k-1}\right) \mathbf{P}_{k-1 \mid k-1} \mathbf{f}^{\prime}\left(\hat{\mathbf{x}}_{k-1 \mid k-1}\right)^{T}+\mathbf{g}^{\prime}\left(\hat{x}_{k-1 \mid k-1}\right) \mathbf{Q}_{k} \mathbf{g}^{\prime}\left(\hat{\mathbf{x}}_{k-1 \mid k-1}\right)^{T},
\end{aligned}
$$

where $\mathbf{f}^{\prime}(\cdot)$ is the gradient of $\mathbf{f}(\cdot)$ with respect to $\mathbf{x}$ and $\mathbf{g}^{\prime}(\cdot)$ is the gradient of $\mathbf{f}(\cdot)$ with respect to $\mathbf{w}$, which are as

$$
\begin{aligned}
\mathbf{f}^{\prime}\left(\hat{\mathbf{x}}_{k-1 \mid k-1}\right) & =\left(\left.\frac{\partial}{\partial \mathbf{x}} \mathbf{f}(\mathbf{x}, \mathbf{w})\right|_{\mathbf{x}=\hat{\mathbf{x}}_{k-1 \mid k-1}, \mathbf{E}(\mathbf{w})=0}\right), \\
\mathbf{g}^{\prime}\left(\hat{\mathbf{x}}_{k-1 \mid k-1}\right) & =\left(\left.\frac{\partial}{\partial \mathbf{w}} \mathbf{f}(\mathbf{x}, \mathbf{w})\right|_{\mathbf{x}=\hat{\mathbf{x}}_{k-1 \mid k-1}, \mathbf{E}(\mathbf{w})=0}\right) .
\end{aligned}
$$

The new prior distribution, $\mathcal{N}\left(\hat{\mathbf{x}}_{k-1 \mid k}, \mathbf{P}_{k-1 \mid k}\right)$, is computed based on the previous Gaussian posterior $\mathcal{N}\left(\hat{\mathbf{x}}_{k-1 \mid k-1}, \mathbf{P}_{k-1 \mid k-1}\right)$. The next step is the measurement update which updates the prior and is performed whenever a measurement is avail- 
able as

$$
\begin{aligned}
\hat{\mathbf{y}}_{k} & =\mathbf{h}\left(\hat{\mathbf{x}}_{k \mid k-1}\right), \\
\varepsilon_{k} & =\mathbf{y}_{k}-\hat{\mathbf{y}}_{k}, \\
\mathbf{S}_{k} & =\mathbf{h}^{\prime}\left(\hat{\mathbf{x}}_{k \mid k-1}\right) \mathbf{P}_{k \mid k-1} \mathbf{h}^{\prime}\left(\hat{\mathbf{x}}_{k \mid k-1}\right)^{T}+\mathbf{R}_{k}, \\
\mathbf{K}_{k} & =\mathbf{P}_{k \mid k-1} \mathbf{h}^{\prime}\left(\hat{\mathbf{x}}_{k \mid k-1}\right)^{T} \mathbf{S}_{k}^{-1}, \\
\hat{\mathbf{x}}_{k \mid k} & =\hat{\mathbf{x}}_{k \mid k-1}+\mathbf{K}_{k} \varepsilon_{k}, \\
\mathbf{P}_{k \mid k} & =\left(\mathbf{I}-\mathbf{K}_{k} \mathbf{h}^{\prime}\left(\hat{\mathbf{x}}_{k \mid k-1}\right)\right) \mathbf{P}_{k \mid k-1},
\end{aligned}
$$

where $\mathbf{h}^{\prime}(\cdot)$ is the Jacobian of the $\mathbf{h}(\cdot)$ function

$$
\mathbf{h}^{\prime}\left(\hat{\mathbf{x}}_{k \mid k-1}\right)=\left(\left.\frac{\partial}{\partial \mathbf{x}} \mathbf{h}(\mathbf{x}, \mathbf{e})\right|_{\mathbf{x}=\hat{\mathbf{x}}_{k \mid k-1}, \mathbf{E}(\mathbf{e})=0}\right) .
$$

The EKF algorithm starts with providing the time update phase and assigning the initial value for the states and the covariance matrix similar to KF. That is, $\hat{\mathbf{x}}_{0 \mid 0}=\mathbf{x}_{0}$ and $\mathbf{P}_{0 \mid 0}=\mathbf{P}_{0}$. As discussed earlier, the linearization in the measurement update at time $k$ is based on the predicted state $\hat{\mathbf{x}}_{k \mid k-1}$. The second order EKF, also known as EKF with bias compensation, is one example where the Hessian of the nonlinear equation is computed at each iteration to achieve a better approximation. One shortcoming of such algorithms is the necessity of higher order differentiations that can cause vastly increased computational complexity and costs. 


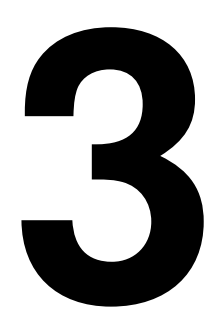

\section{Pedestrian Dead Reckoning Positioning}

Position and orientation information can be obtained by integrating inertial sensor (accelerometer and gyroscope) measurements. The integration process is called Dead Reckoning (DR). For example, the processing phase can be advancing the previously determined position over elapsed time based on known or estimated speed. The main advantage of DR is that it is independent of extra infrastructures and radio signals. This allows DR to be applied in many navigation applications such as pedestrian navigation and localization, marine navigation, automotive and autonomous navigation.

The price of being independent of extra infrastructures is the well-known disadvantage of all pure dead reckoning techniques; the cumulative errors. This makes pure DR methods become infeasible for most purposes while radio signalbased navigational systems provide highly accurate position information. For example, GPS-based navigation systems can provide position estimates with few meters accuracy. These methods, however, rely on the received signals and are prone to erroneous estimates in poor SNR conditions or even failure in environments where the signal is blocked [3].

Nowadays, ubiquitous smartphones are embedded with inertial sensors such as accelerometer, gyroscope, and magnetometer. The sensors can be used for pedestrian navigation by means of dead reckoning. For navigational purposes, the accelerometer can be used as a pedometer to detect the steps and traveled distance and the built-in magnetometer as a compass heading provider. The gyroscope also provides information about how the phone is carried. Pedestrian dead reckoning (PDR) can be considered as an add-on to the other navigation methods in a similar way to automotive navigation or extend the navigation techniques into areas where other navigation systems are unavailable.

Fusing all pieces of information received from IMU sensors, it is possible to have a simple PDR implementation given that the subject holds an IMU unit in 
the hand in front of the body and walks with constant speed. The magnetometer measurements can be transformed into directional information and each step causes the position to move forward a fixed distance in the estimated direction. However, there are many challenges that must be considered in order to have more accurate estimates. The accuracy of the algorithm is limited by the sensor precisions and magnetic disturbances. In addition to hardware and environmental factors, the algorithm design parameters need to be carefully tuned. For instant, how the algorithm detects a step from sensor measurements depends on a threshold that is set on signal magnitude. How this threshold is defined together with other design algorithms such as unknown gait parameters, like the length of each step, unknown phone position, the way that the phone is being carried, and user's motion mode identification are all among the challenges of PDR that are studied in detail in this chapter and Chapters 5 and 6.

In Section 3.1 of this chapter, we first describe all sensors whose measurements are used in this thesis for positioning and navigational purposes. Then, Section 3.3 presents general odometric models for PDR and introduces supporting measurements used to increase accuracy and reliability of the system.

\subsection{Sensors}

Depending on the application, different types of sensors can be used to provide the desired information used for positioning. The sensed and reported quantity relates to the parameter or state of interest that is to be estimated. For example, in autonomous cars, sensors can be placed such that they provide information about the position and orientation of the car. Similarly, sensors can be used to obtain information about position and orientation of people that can further be applied to PDR algorithms.

Two kinds of sensors are commonly used in localization problems. One set of sensors measure those quantities that are indirectly related to the subject. The magnetometer is an example of this type measuring the surrounding magnetic field. The directional information can be inferred from the measurements. Other examples are cameras filming the surrounding, etc. The second set of sensors, measure values that are directly related to the subject. For example, the movement of the subject, orientation, etc. Inertial sensors for instance measure angular rate and acceleration that can be used for pose estimation.

Significant developments of IMUs in recent years have enhanced their accuracy, made them smaller, and lowered their price. This allows easy access to these units as either embedded in almost all recent smartphones or as dedicated devices. Fig. 3.1 is an example of a sensor produced by Xsens which contains IMU sensors. We first describe the three main IMU sensors that are mainly used for positioning purposes. In this thesis, we use the accelerometer and gyroscope sensors for estimating the PDR parameters and position estimation. In some applications, it might be necessary to use a magnetometer as a complementary sensor in addition to accelerometer and gyroscope [52]. 


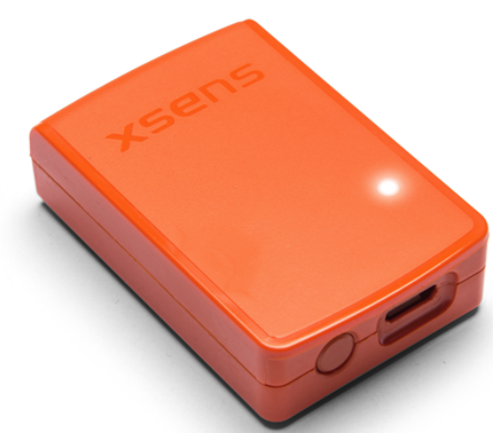

Figure 3.1: An Xsens motion sensor containing an IMU. Courtesy of Xsens Technologies.

\section{Accelerometer}

Accelerometers provide accelerations which can be used to define an estimate of the position of the sensor. In accelerometers, the quantity that is measured is the specific force acting on the sensor. Both the earth's gravity and the sensor's acceleration contribute to the measured force by the sensor. However, the sensor's acceleration has generally much smaller value compared to the earth's gravity, $g=9.81 \mathrm{~m} / \mathrm{s}^{2}$.

The earth's gravity will, therefore, form a large contribution of the measured value by the accelerometer, while the motion of the sensor has a relatively small contribution. Subtracting the earth's gravity, the reported value is the non gravitational force. The accelerometer measurement model is given as [84]

$$
y_{k}^{\mathrm{acc}}=\mathbf{Q}\left(\mathbf{q}_{k}\right)(a-\mathbf{g})+b_{k}^{\mathrm{acc}}+e_{k}^{\mathrm{acc}},
$$

where $\mathbf{g}=[0,0, g]^{T}$ is the gravitation vector in Earth fixed condition, $a$ is the sensor's acceleration. $b_{k}^{\text {acc }}$ denotes accelerometer bias, and $e_{k}^{\text {acc }}$ is the magnetometer measurement noise. $\mathbf{Q}$ and $\mathbf{q}_{k}$ are the rotation matrix and unit quaternions, respectively. Unit quaternions use a 4-dimensional description of the orientation. More details about orientation representations is presented in [52].

\section{Gyroscope}

Gyroscopes provide angular rates which can be used to define an estimate of the orientation of the sensor. The measured value is the angular velocity, i.e. rate of turn. By integrating of the signal allows for adding up the changes in orientation over time. The gyroscope measurement model is given by [84]

$$
y_{k}^{\text {gyro }}=\omega_{z, k}+b_{k}^{\text {gyro }}+e_{k}^{\text {gyro }} \text {, }
$$


where $\omega_{z, k}$ is the yaw rate which is the gyroscope measurement in the horizontal plane, $b_{k}^{\text {gyro }}$ denotes gyroscope bias, and $e_{k}^{\text {gyro }}$.

The accuracy of measurements is a trade off between size and price of the sensor. For example, optical gyroscopes provide precise measurements but are hard to reduce much in size. Another technology used for producing gyroscope is the Micro-Electro-Mechanical Systems (MEMS) aimed to create smaller devices. MEMS gyroscopes all rely on the same principle, that of vibrating objects undergoing rotation.

\section{Magnetometer}

Measurements provided by a magnetometer have contributions both from the local earth magnetic field and all other magnetic material producing local disturbances. In cases where the earth's field is stronger than local and mostly time varying-disturbances, information about magnetic north, thus the geographical north, can be inferred from the measurements. Magnetometers therefore, typically serve the purpose of a compass and provide useful information about the sensor's heading. The magnetometer measurement model is given as [84]

$$
y_{k}^{\operatorname{mag}}=\mathbf{Q}\left(\mathbf{q}_{k}\right) m^{0}+e_{k}^{\operatorname{mag}},
$$

where $m^{0}$ is the earth magnetic field in world coordinates. $e_{k}^{\text {mag }}$ is the magnetometer measurement noise. $\mathbf{Q}$ and $\mathbf{q}_{k}$ are the rotation matrix and unit quaternions, respectively.

Magnetometers can be used as complementary sensors to deal with biased and noisy measurements of accelerometer and gyroscopes. Integration of the angular velocity to obtain orientation and then integrating the acceleration to estimate the position introduce integration drift as a result of the noisy measurements provided by inertial sensors.

\subsection{Sensor Fusion}

Estimating properties of interest, $\mathbf{x}_{k}$, using some or all of the measurements $\mathbf{y}_{k}$ introduced above, is a sensor fusion problem. The process model, defining how $\mathbf{x}_{k}$ evolves over time, and measurement model, defining how $\mathbf{y}_{k}$ relates to $\mathbf{x}_{k}$, are used to infer the properties $\mathbf{x}_{k}$ from the measurements $\mathbf{y}_{k}$. Often, $\mathbf{x}_{k}$ is called state and represents the sought system property. States to be estimated can correspond to a wide variety of quantities from unknown constant properties such as weight to time varying position or orientation of a unit or even the surrounding environment. Orientation of the unit together with its position and velocity are the typical components of the states in this thesis.

One way to estimate each entity of the state vector $\mathbf{x}_{k}$ is to use information given by the sensors individually. Another alternative, used in the sensor fusion framework, is to fuse all the information obtained from inertial sensors, magnetometers, and GPS measurements and filter them to get the joint estimate of all 


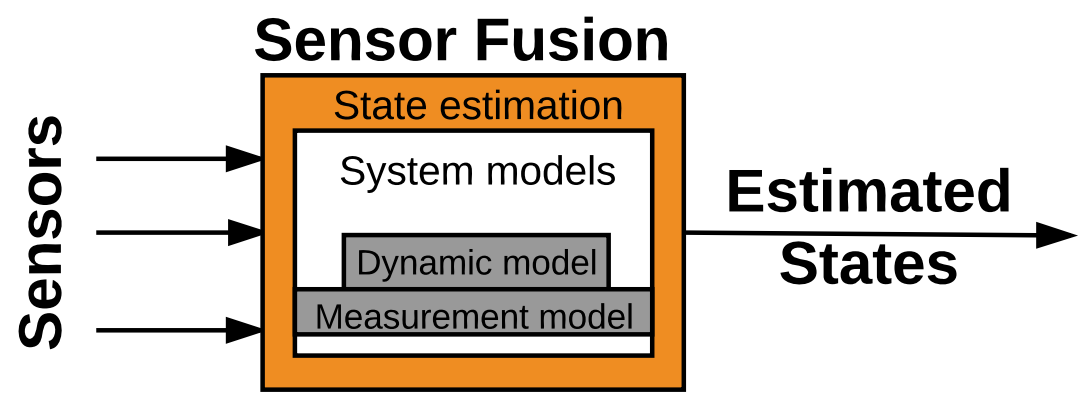

Figure 3.2: Illustration of a sensor fusion framework [15].

entities of the states $\mathbf{x}_{k}$. The output of the filter is both the estimated states $\hat{\mathbf{x}}_{k}$ and a measure of how uncertain the estimates are.

Applying sensor fusion algorithms to obtain the joint estimate $\hat{\mathbf{x}}_{k}$ leads to performance improvements compared to the estimates obtained by each sensor. Evaluation of the performance is based on multiple criteria such as more certain and robustness and number of sensors needed.

Fig 3.2 illustrates a generic overview of the fusion algorithm. For the framework to provide $\hat{\mathbf{x}}_{k}$, three components are needed; measurements $\mathbf{y}_{k}$ that relate to the system states $\mathbf{x}_{k}$ through measurement models, model of the system dynamics, and a state estimation system that provides $\hat{\mathbf{x}}_{k}$. In this framework, rather than treating each sensor measurement individually, all measurements from all sensors, either of the same type or measurements of different types, are used as the input to the state estimation box.

\subsection{Model Framework}

The generic state vector used in PDR problems consists of three components

$$
\mathbf{x}_{k}=\left(\begin{array}{l}
\mathrm{x}_{k} \\
\mathrm{y}_{k} \\
\psi_{k}
\end{array}\right) \text {, }
$$

where $x_{k}$ and $y_{k}$ are the Cartesian position and $\psi_{k}$ is the heading, see [35]. The input signals are given as

$$
\mathbf{u}_{k}=\left(\begin{array}{c}
v_{k} \\
\dot{\psi}_{k}
\end{array}\right)
$$




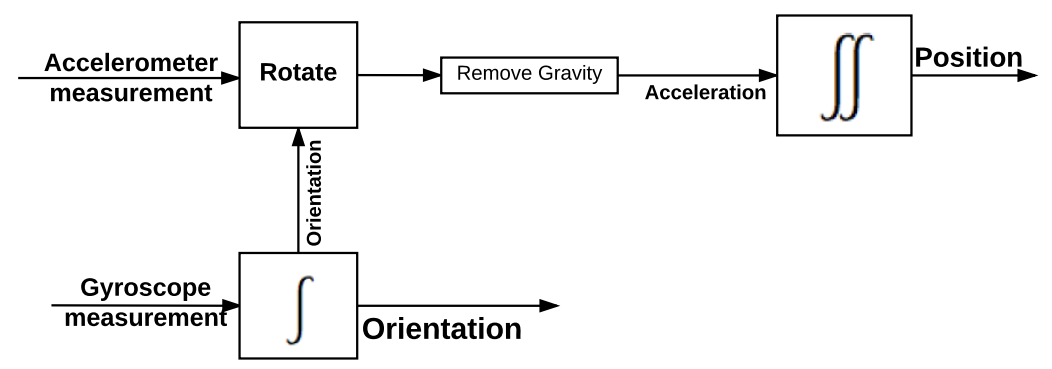

Figure 3.3: Using the accelerometer and gyroscope to estimate the position and orientation. The initial values for the position and orientation are assumed as known value [52].

where $v_{k}$ is the velocity and $\dot{\psi}_{k}$ is the yaw rate. The dynamic model are described by

$$
\begin{aligned}
\mathrm{x}_{k+1} & =\mathrm{x}_{k}+T v_{k} \cos \left(\psi_{k}\right), \\
\mathrm{y}_{k+1} & =\mathrm{y}_{k}+T v_{k} \sin \left(\psi_{k}\right), \\
\psi_{k+1} & =\psi_{k}+T \dot{\psi}_{k},
\end{aligned}
$$

where $T$ is the sampling interval. These inertial signals are given by sensors either mounted on or being carried by the subject. Depending on the application, other components might be added to the generic state vector (3.4). Typically, magnetometer readings are used as measurements whose model is given in (3.3).

Measurements signals from both a tri-axial accelerometer and a tri-axial gyroscope sensors are used for position and PDR parameters estimations. The integrating these signals gives position and orientation of the device equipped with the IMUs as illustrated in Fig. 3.3 shows. As this figure suggests, the position of the sensor is estimated by double integration of the acceleration signal without the contribution of the earth's gravity. This requires subtracting the earth's gravity from the accelerometer measurements. Thus, to do the subtraction, the orientation of the device needs to be known a priori. Hence, when inertial sensors are used to estimate the position, the estimation of the orientation is the first step.

As Fig. 3.3 shows, there are two integration steps to estimate the sensor's position and orientation using inertial sensors. The drift introduced by integrating measurement errors degrades the accuracy of position and orientation estimation considerably. Consequently, complementary measurements need to be used together with inertial sensors' measurement to improve the estimation quality. Subsequently, different supporting measurements such as GPS outdoor, angle dependent RSS outdoor, RSS fingerprint maps indoor, and proximity sensors are added to the measurement model to provide more accurate estimation. The state vector is also application dependent and the generic state (3.4) is extended by other components accordingly. For example, one might add the travelled dis- 
tance, length of a taken step, threshold for step detection, etc. In the subsequent chapters, various problem formulations leading to an extended state vector will be described in more details.

\subsection{Supporting measurements}

Supporting measurements could be obtained by additional sensors and/or receiver antennas that the mobile device is equipped with. They are supposed to improve both the accuracy and reliability of the positioning algorithms. These supportive measurements, however, might not necessarily be synchronized with the IMU. Further, they might not always be available during the entire localization process. Therefore, they will be included in the measurement model whenever they are available.

\subsubsection{GPS outdoor}

The Global Positioning System is the most well-known Global navigation satellite system (GNSS). The accuracy of the GPS receivers found in smartphones is around 5 meters [29]. GPS receivers use multiple satellites and trilateration to determine the position and time of a user.

GPS satellites continuously transmit signals down to the Earth over dedicated radio frequencies. The GPS receiver, among other data, receives a time stamp from the satellites momentary visible, along with satellite ephemeris data which contains the satellites positions in the sky. The GPS receiver antenna can then accurately calculate its position and time if it hears at least four satellites [62].

The GPS uses the World Geodetic System (WGS84) as its reference coordinate system [28]. By converting the Geodetic, WGS84, to Cartesian East-North-Up (ENU) coordinates, the estimated position can be either fused to the measurement model directly or used to calculate the speed.

Accurate position (and time) estimation using GPS measurements, however, is not guaranteed when the received satellite signals are weak. This is the shortcoming of GNSS systems and can lead to poor position estimates or even a failure.

\subsubsection{RSS Outdoor}

Wireless networks can be used as a localization infrastructure in various ways. The received signal strength, knowing the signal strength transmitted by the sender, can be translated to distance between the sender and the receiver and further used to estimate the propagation parameters as well as the radio's position.

The received signal is stronger close to the sender than in further distances. The average of the received signal for free space propagation can be modeled as a function of the distance between sender and receiver [69],

$$
\operatorname{Pr}=P_{0} K\left(\frac{d}{d_{0}}\right)^{-B},
$$


The RSS (in $\mathrm{dBm}$ ) is given as

$$
\operatorname{Pr}(d B m)=10 \log P_{r}=10 \log P_{0}+10 \log K-10 B \log \frac{d}{d_{0}},
$$

where $d$ is the distance from the receiver to the transmitter, in meters and $d_{0}$ is the reference distance point. The degree of attenuation is given by a path loss exponent $B$ and $K$ is a unit-less constant that depends on the antenna characteristics. Moreover, it can also be shown that the average of the large-scale path loss from the receiver to the transmitter is a function of distance by using a path loss exponent, $B$ as

$$
L(d) \propto\left(\frac{d}{d_{0}}\right)^{B}
$$

and can be expressed in $\mathrm{dB}$ as

$$
L(d)=L\left(d_{0}\right)+10 B \log \frac{d}{d_{0}},
$$

where $L\left(d_{0}\right)$ is the reference path loss, selected based on the propagation environment. For notational convenience, $L\left(d_{0}\right)$ is denoted by $A$ in the sequel. For more details consult [69].

In a cellular system, with known base station positions, the position of the receiver can be estimated using the approximated distance. That is, the receiver is located on a circle with the radius of the approximated distance $d$ around the base station. Using multiple base stations, we can estimate the exact position through lateration. This technique can be used in an open space or outdoor environment

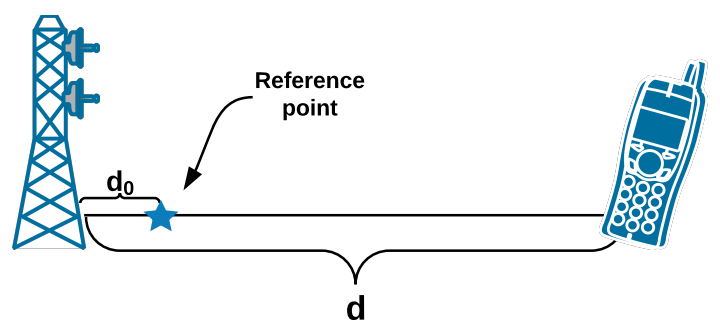

Figure 3.4: Free space propagation.

for determining a mobile user position. However, indoor environments are more difficult as the channel cannot be modeled easily. Typically, in indoor scenarios, RSS is used in combination with fingerprinting.

\subsubsection{RSS Fingerprint Maps Indoor}

The fingerprinting approach based on Wi-Fi has become an interesting research topic based on 2-D modeling $[46,87]$. This positioning solution, stores RSS of 
specific locations in a fingerprint database. These signal fingerprints are then matched to measured RSS at the user's location to estimate its position. In this way, fingerprinting determines the location of the user by comparing the obtained RSS values to a radio map.

The final position estimate, in this method, is provided in two phases as illustrated in Fig. 3.5. The radio map is formed in the first, offline, phase of the fingerprinting method. In this phase, a database containing spatial reference information together with their environmental radio characteristics are gathered to form the radio map. In the second, online, phase, the user device measures the same radio characteristics at its current location and the results are compared to the stored values.

The accuracy of this method highly depends on the radio map acquired using the offline survey of the environment. A precise radio map can make this approach very efficient. The need for a precise radio map is, however, the main disadvantage of the fingerprinting approach as a setup time and costly signal strength system calibration is required. Additionally, the scalability can also be a problem as the obtained fingerprints of a wide area requires a high data volume to be managed. Finally, configuration properties such as the initial location of the access points needs to remain fixed otherwise a new database needs to be created. Many researchers have addressed these issues to improve fingerprint matching technology across all aspects [27, 23, 89].

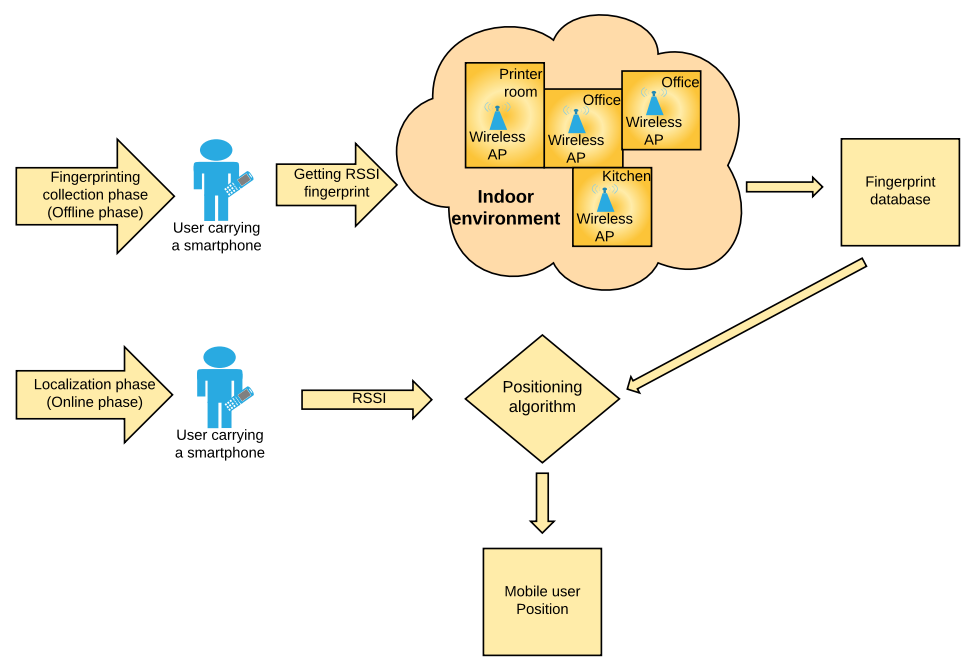

Figure 3.5: Fingerprinting based positioning [63].

\subsubsection{Proximity Sensors}

Proximity sensors are capable of sensing the environment and detecting objects in their vicinity by emitting electromagnetic signals. A proximity sensor flashes 
out a beam of electromagnetic radiation and monitors reflections. Any sensed reflection would then imply the existence of an object in the vicinity. The emitted signals from proximity sensors are of different types depending on the used technology.

Indoor wireless localization industry benefits from these sensors mostly upon the release of the Bluetooth-Low-Energy (BLE) protocol when Bluetooth beacon proximity sensors are introduced. The long scan time of traditional Bluetooth $(\sim$ $10 \mathrm{~s})$ was a limiting factor, this has been overcome by the new BLE technology which is supported by most current devices. The light weight of BLE beacons together with their small size and low cost may increase their potential to become a dominant wireless localization technology [22].

Indoor localization can be performed by using periodic or event-triggered RSS measurements provided by BLEs [25]. Based on a pre-defined threshold $P_{t h}$, proximity information in the network can be obtained as [25]

$$
\text { Proximity } \triangleq \begin{cases}0, & R S S \leq P_{t h} \\ 1, & R S S>P_{t h}\end{cases}
$$

Such reports can then be utilized as an indicator of a target in the vicinity. Knowing the coverage range of the sensor allows for narrowing down the search area for the target's location. That is, the exact location estimate of the target can be found in a second stage by only considering the, rather small, area estimated using the proximity sensor. 


\section{Part II}

\section{Applications}





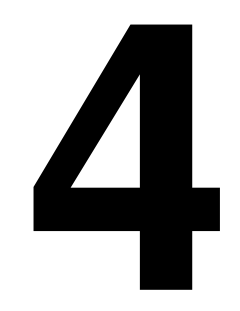

\section{Antenna Gain Parameter Estimation}

The methods introduced in Section 2.1 are used to solve the parameter estimation problem introduced in this chapter. We present a method to jointly estimate the antenna gain and propagation model parameters using positioned RSS measurements. This application, its related background theory and the proposed solution were previously published by the author in (FUSION2015) [47, C2015 IEEE].

A semi-parametric model for RSS measurements is introduced that can be used to predict the base station antenna coverage in cellular radio networks. The model is composed of an empirical log-distance model and a deterministic antenna gain model that accounts for possible non-uniform base station antenna radiation. A least-squares estimator is proposed to jointly estimate the path loss and antenna gain model parameters. Simulations as well as experimental results verify the efficacy of this approach. The method can provide improved accuracy compared to conventional path loss based estimation methods.

This chapter starts with introducing a semi-empirical model for RSS measurement in Section 4.1. Then, Section 4.2 addresses requirements for the joint propagation model and antenna parameter estimation representation. Section 4.3 evaluates the performance of the proposed model using simulations. Finally, the results obtained by applying the method on real-field data are given in Section 4.4.

\subsection{RSS Measurement Model}

The RSS measurement can be formulated in the form (2.1). For convenience, we restate it in a general form as

$$
y=\mathbf{h}(\mathbf{u}, \boldsymbol{\theta})+e,
$$

where $\mathbf{h}(\mathbf{u}, \boldsymbol{\theta})$, in this scenario, is a propagation model as a function of both position $\mathbf{u}$ and unknown parameters $\theta$. $\theta$ describes the radio channel that accounts 
for propagation effects, such as attenuation, diffraction, or reflection, that the electromagnetic wave is affected by, when traveling between the mobile station (MS) and the base station (BS). To clarify, u denotes a known (vector) variable providing relative position dependent information of the MS and the BS and/or the environment, $\theta$ is the unknown deterministic antenna and propagation parameters vector and $e$ represents a statistical noise term (here assumed to be additive) that accounts for effects that cannot be captured by the propagation model.

The propagation models can be broadly categorized into three types; deterministic, empirical, and semi-empirical models [14]. The deterministic models are based on techniques such as ray tracing or ray launching that require accurate knowledge of the environment such as high resolution building data. These models are very accurate, but also the most complex ones. Empirical models use heuristic equations that have been derived from extensive measurement campaigns. These models are very simple, but less accurate than their deterministic counterparts. Among the most popular empirical models are the Okumura-Hata and COST 231 models [14, 36, 18]. Semi-empirical models are composed of both deterministic and empirical models and provide a good compromise between accuracy and complexity.

In the following, we introduce a semi-empirical propagation model for the RSS measurement. It combines an empirical distance-dependent propagation loss (or path loss) model $L(\mathbf{u})$ with a deterministic model $G_{\text {ant }}(\mathbf{u})$ representing the possible non-uniform radiation of the BS antenna with respect to the MS position (antenna gain model). We further make the common assumption that the RSS measurement is time-averaged, such that temporal effects resulting from smallscale fading can be neglected. Hence, the proposed semi-empirical model for the RSS measurement in logarithmic scale $(\mathrm{dBm})$ can be written as

$$
y=P_{T}-\left\{L(\mathbf{u})-G_{\text {ant }}(\mathbf{u})\right\}+e,
$$

where $\mathbf{u}$ is the vector holding the relative position information of the MS with respect to the BS antenna, $P_{T}$ is the BS transmit power in $\mathrm{dBm}$, and $e$ is a statistical term which accounts for the errors resulting from quantization, slow fading and other effects that are not captured by the propagation model. The error term $e$ is modeled with a zero-mean Gaussian distribution with variance $\sigma^{2}$.

\subsubsection{Path Loss Model}

A path loss model presents signal attenuation in space. In this work, the logdistance model is used as it forms the basis of most models available in the literature $[69,46]$. The log-distance model is given by

$$
L(d)=A+10 B \log _{10}\left(\frac{d}{d_{0}}\right),
$$

where $A$ is the reference path loss, $B$ is the path loss exponent, $d$ is the Euclidean distance between the MS and BS, and $d_{0}$ represents the distance at which the reference path loss $A$ is determined. The value of $d_{0}$ generally depends on the cell size, and values that can be typically found are $100 \mathrm{~m}$ or $1 \mathrm{~km}$. 


\subsubsection{Antenna Gain Model}

Base station antenna modeling mainly concerns crude models that capture the far-field (at some distance from the antenna) gain in various directions. Models, that have become popular in recent years are separated into one horizontal plane model $G_{\mathrm{h}}(\phi)$ and one vertical plane model $G_{\mathrm{v}}(\varphi)$, and the combined antenna gain is merely the two model contributions added together in logarithmic scale according to

$$
G_{\text {ant }}(\phi, \varphi)=G_{\mathrm{h}}(\phi)+G_{\mathrm{v}}(\varphi) .
$$

Simplified models neglect the vertical component and model only the horizontal antenna gain. In this work, we consider the antenna gain model proposed in [30] and that is also adopted for the radio network evaluations in 3GPP. The horizontal gain model is given by

$$
G_{\mathrm{h}}(\phi)=G_{\max }-\min \left(12\left(\frac{\phi-\phi_{0}}{\phi_{\mathrm{h}}}\right)^{2}, G_{\mathrm{h}, \min }\right),
$$

where $G_{\max }$ denotes the maximum antenna gain in $\mathrm{dBi},-180^{\circ}<\phi \leq 180^{\circ}$ is the antenna azimuth angle defined in the $x y$-plane, counted counter-clockwise from the positive $x$-axis, $\phi_{\mathrm{h}}$ is the antenna's horizontal bandwidth in degree, which represents the bandwidth at which the antenna gain is half of the maximum gain (also known as half-power beamwidth), $\phi_{0}$ is the antenna angle in degree pointing into the direction of maximum gain (antenna boresight angle), and $G_{\mathrm{h} \text {,min }}$ denotes the front-to-back ratio measured in $\mathrm{dB}$, given the relative difference between antenna beam direction gain $G_{\mathrm{h}}\left(\phi_{0}\right)=G_{\max }$ and the backlobe gain $G_{\mathrm{h}}\left(\phi_{0}+\right.$ $\left.180^{\circ}\right)=G_{\max }-G_{\mathrm{h}, \min }$. The vertical antenna gain is modeled with

$$
G_{\mathrm{v}}(\varphi)=\max \left(-12\left(\frac{\varphi-\varphi_{\text {etilt }}}{\varphi_{\mathrm{v}}}\right)^{2}, G_{\mathrm{v}, \min }\right),
$$

where $-90^{\circ}<\varphi \leq 90^{\circ}$ is the negative antenna elevation angle relative to the horizontal plane, i.e. $\varphi=90^{\circ}$ is downwards, $\varphi=0^{\circ}$ is along the horizontal plane, and $\varphi=-90^{\circ}$ is upwards. The angle $\varphi_{\text {etilt }}$ given in degree is the electrical antenna downtilt that models the angle downwards from the horizontal plane at which the antenna is electrically directed, $\varphi_{\mathrm{v}}$ is the antenna's half-power beamwidth in the vertical direction, and $G_{\mathrm{v}, \mathrm{min}}$ is the side lobe level in $\mathrm{dB}$ of the vertical pattern that represents the side lobe gain level in relation to the antenna vertical beam direction gain. As an example, Fig. 4.1 illustrates the horizontal antenna gain model (4.5), together with real data from an antenna, see [30] for an additional example including the vertical antenna gain model.

\subsection{Joint Path Loss and Antenna Parameter Estimation}

The joint contribution of the attenuation due to path loss and the antenna gain enter the measurement equation additively. Hence identification of some param- 


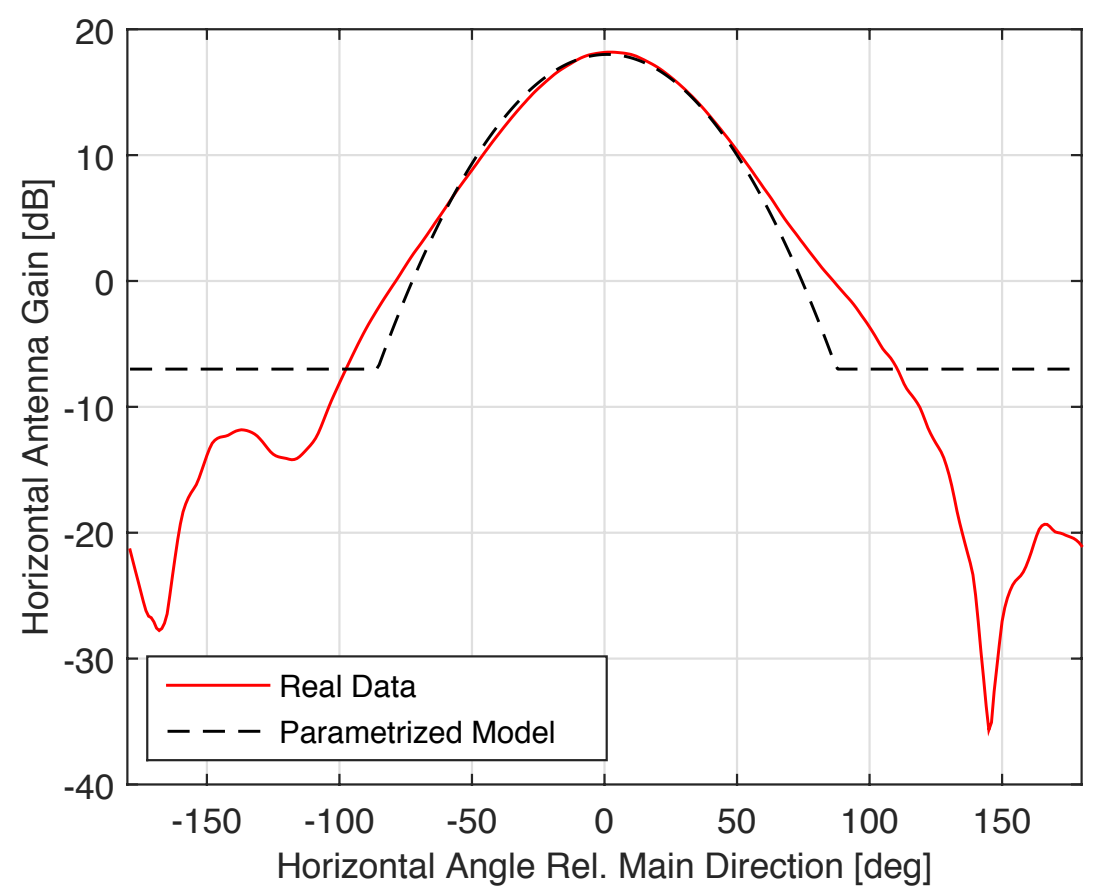

Figure 4.1: Illustration of horizontal antenna gain pattern [47, C2015 IEEE].

eters is not possible due to unobservability. For instance, it is not possible to estimate the reference path loss $A$ and the maximum antenna gain $G_{\text {max }}$ separately. Another issue with the joint parameters estimation is that the antenna gain is modeled different inside and outside the main lobe using $\min ($, ) and $\max ($, ) functions which results in non-linear equations. Under the assumption that the main lobe components are dominant, the contribution of the antenna gain simplifies to

$$
\tilde{G}_{\text {ant }}(\phi, \varphi)=G_{\max }-12\left(\frac{\phi-\phi_{0}}{\phi_{\mathrm{h}}}\right)^{2}-12\left(\frac{\varphi-\varphi_{\mathrm{etilt}}}{\varphi_{\mathrm{v}}}\right)^{2} .
$$

With this simplification, the maximum likelihood estimate of the parameters becomes tractable, and can be found according to

$$
\hat{\boldsymbol{\theta}}^{\mathrm{ML}}=\arg \max _{\boldsymbol{\theta} \in \Theta} p(\mathbf{y} ; \boldsymbol{\theta}),
$$

where $\mathbf{y}$ is an independent and identically distributed (i.i.d.) sequence of $m$ RSS measurements and $\theta$ are the unknown parameters. Under the Gaussian noise assumption the maximum likelihood solution is equivalent to the least squares estimator. The RSS measurement becomes linear in the following unknown parameters $\theta=\left[A, B, \phi_{\mathrm{h}}^{-2}, \varphi_{\mathrm{v}}^{-2}\right]^{T}$, where it has been assumed that the antenna main direction $\phi_{0}$, the electrical downtilt $\varphi_{\text {etilt }}$, the transmission power $P_{T}$ and the 
maximum antenna gain $G_{m}$ all are known. The least squares estimate is given as

$$
\hat{\boldsymbol{\theta}}^{\mathrm{LS}}=\hat{\boldsymbol{\theta}}^{\mathrm{ML}}=\left(\mathbf{H}^{T} \mathbf{H}\right)^{-1} \mathbf{H}^{T} \mathbf{Z},
$$

where

$$
\mathbf{H} \triangleq\left[\begin{array}{cccc}
1 & 10 \log _{10}\left(\frac{d_{1}}{d_{0}}\right) & 12\left(\phi_{1}-\phi_{0}\right)^{2} & 12\left(\varphi_{1}-\varphi_{\text {etilt }}\right)^{2} \\
\vdots & \vdots & \vdots & \vdots \\
1 & 10 \log _{10}\left(\frac{d_{m}}{d_{0}}\right) & 12\left(\phi_{m}-\phi_{0}\right)^{2} & 12\left(\varphi_{m}-\varphi_{\text {etilt }}\right)^{2}
\end{array}\right],
$$

and

$$
\mathbf{Z} \triangleq\left[\begin{array}{c}
P_{\mathrm{T}}+G_{\max }-y_{1} \\
\vdots \\
P_{\mathrm{T}}+G_{\max }-y_{m}
\end{array}\right]
$$

An unbiased estimate of the noise variance $\hat{\sigma}^{2}$ is given by

$$
\hat{\sigma}^{2}=\frac{1}{m-1} \sum_{i=1}^{m}\left(\hat{z}_{i}-z_{i}\right)^{2},
$$

where $\hat{z}_{i}$ 's are found by plugging in the least squares solution into $\hat{\mathbf{Z}}=\mathbf{H} \hat{\boldsymbol{\theta}}^{\mathrm{LS}}$. As already stated earlier, the BS transmit power $P_{\mathrm{T}}$ and maximum antenna gain $G_{\max }$ can not be estimated separately from the path loss parameter $A$. Therefore, it is reasonable to assume them a priori known, or to approximate them using nominal values taken from antenna specification documents, or by lumping these parameters together forming the new (unknown) parameter $\tilde{A}=P_{\mathrm{T}}-A+G_{\text {max }}$, that is to be estimated instead.

For the main lobe assumption to hold, the horizontal and vertical angles needs to be close to the antenna main beam direction. This implies the following boundary conditions to be satisfied

$$
\begin{array}{r}
\left|\phi-\phi_{0}\right|<\phi_{\mathrm{h}} \sqrt{\frac{G_{\mathrm{h}, \mathrm{min}}}{12}}, \\
\left|\varphi-\varphi_{\text {etilt }}\right|<\varphi_{\mathrm{v}} \sqrt{\frac{-G_{\mathrm{v}, \mathrm{min}}}{12}} .
\end{array}
$$

Clearly, the boundary conditions depend on the unknown parameters $\phi_{\mathrm{h}}$ and $\phi_{\mathrm{v}}$, making it difficult to motivate considering only those RSS measurements in the estimation process that fulfill the above requirements. However, it is always possible to use smaller values for the parameters in (4.13) deviating from the nominal values that can be typically found in antenna specification documents, implying a smaller main lobe area from which RSS measurements can be used in the estimation process. However, these parameters should be carefully selected in order to avoid deteriorating the estimation results, for instance by assuming a too small main lobe area. 


\subsection{Simulated Data Experiments}

In this section, the performance of the algorithm proposed in the previous section is tested on simulated data. The RSS measurements are generated from (4.2) using the parametrized antenna gain model, see (4.5) and (4.6). In Table 4.1, the path loss and antenna gain model parameters are listed that have been used in the simulations. The antenna parameters correspond to typical values that can be found in antenna specifications. The path loss exponent $B$ is typically between 2 (free-space propagation) and 4 (dense urban environment) and has been chosen slightly above the free-space propagation, in order to better reflect outdoor BS deployments in rural areas. For the antenna parameters given in Table 4.1 , it is possible to derive the boundary conditions (4.13), where RSS measurements shall be collected in order to not violate the simplified antenna gain model assumption (4.7). It is easy to show that for an antenna with the param-

Table 4.1: List of path loss and antenna model parameters [47, C2015 IEEE].

\begin{tabular}{|c||c||c|}
\hline Parameter & Description & Value \\
\hline$P_{\mathrm{T}}$ & BS transmit power & $32 \mathrm{dBm}$ \\
\hline$A$ & Reference path loss & $100 \mathrm{~dB}$ \\
\hline$B$ & Path loss exponent & $2.3 \mathrm{~dB}$ \\
\hline$d_{0}$ & Reference Distance & $1000 \mathrm{~m}$ \\
\hline$\sigma$ & Error standard deviation & $4 \mathrm{~dB}$ \\
\hline$G_{\max }$ & Maximum gain & $18 \mathrm{dBi}$ \\
\hline$\phi_{\mathrm{h}}$ & Horizontal beamwidth & $65^{\circ}$ \\
\hline$\phi_{0}$ & Boresight angle & $0^{\circ}$ \\
\hline$G_{\mathrm{h}, \min }$ & Front-to-back ratio & $30 \mathrm{~dB}$ \\
\hline$\varphi_{\mathrm{etilt}}$ & Vertical downtilt & $9^{\circ}$ \\
\hline$\varphi_{\mathrm{v}}$ & Vertical beamwidth & $7^{\circ}$ \\
\hline$G_{\mathrm{v}, \min }$ & Side lobe level & $-18 \mathrm{~dB}$ \\
\hline$h_{\mathrm{BS}}$ & BS height & $30 \mathrm{~m}$ \\
\hline \multicolumn{2}{|}{}
\end{tabular}

eters given in Table 4.1 , the requirements for the main lobe area are given by $-103^{\circ} \leq \phi \leq 103^{\circ}$ and $0.4^{\circ} \leq \varphi \leq 17.6^{\circ}$, respectively. The azimuth requirement corresponds to more than the entire $120^{\circ}$ sector the antenna normally covers (Note, that in cellular radio networks each cell site is normally equipped with three antennas each covering a $120^{\circ}$ sector). With a relative antenna height of $30 \mathrm{~m}$, this means that the elevation requirements are valid for distances from the BS between $30 \mathrm{~m} / \tan \left(17.6^{\circ}\right) \approx 95 \mathrm{~m}$ and $30 \mathrm{~m} / \tan \left(0.4^{\circ}\right) \approx 4030 \mathrm{~m}$. On the other hand, if we assume smaller values for the half-power beamwidth, e.g. $\phi_{\mathrm{h}}=60^{\circ}$ and $\phi_{\mathrm{v}}=6^{\circ}$, the main lobe area from which RSS measurements could be used in the estimation process, would shrink to $-95^{\circ} \leq \phi \leq 95^{\circ}$ and $1.7^{\circ} \leq \varphi \leq 16.4^{\circ}$, yielding distances from the BS between $102 \mathrm{~m}$ and $1040 \mathrm{~m}$, which is still acceptable.

Based on the above results, i.i.d. RSS measurements are generated by distributing MS positions uniformly within the main lobe area. Here, we distinguish be- 
tween the true boundary conditions and the approximate boundary conditions that have been introduced above. The estimation results are shown in Table 4.2 and 4.3 , respectively.

It can be observed that the estimation results improve as the number of RSS mea-

Table 4.2: Estimation results for true boundary conditions [47, C2015 IEEE].

\begin{tabular}{|l||c|c|c|c|c|}
\hline Parameter & $A[\mathrm{~dB}]$ & $B[\mathrm{~dB}]$ & $\phi_{\mathrm{h}}\left[^{\circ}\right]$ & $\varphi_{\mathrm{v}}\left[^{\circ}\right]$ & $\sigma[\mathrm{dB}]$ \\
\hline \hline True & $\mathbf{1 0 0}$ & 2.3 & $\mathbf{6 5}$ & $\mathbf{7}$ & 4 \\
\hline Est. $(m=1 e 3)$ & 99.46 & 2.23 & 64.88 & 6.83 & 4.07 \\
\hline Est. $(m=2 e 3)$ & 99.86 & 2.27 & 64.92 & 6.93 & 3.96 \\
\hline Est. $(m=5 e 4)$ & 100.37 & 2.33 & 64.99 & 7.11 & 4.03 \\
\hline
\end{tabular}

surements $m$ used in the least-squares solution is increased. It can be also seen that there is essentially no difference whether the true boundary conditions or the approximate boundary conditions are used to select the RSS measurements for the parameter estimation process.

As an example, the estimation results of the path loss parameters are shown in

Table 4.3: Estimation results for approximate boundary conditions [47, (C)2015 IEEE].

\begin{tabular}{|l||c|c|c|c|c|}
\hline Parameter & $A[\mathrm{~dB}]$ & $B[\mathrm{~dB}]$ & $\phi_{\mathrm{h}}\left[{ }^{\circ}\right]$ & $\varphi_{\mathrm{v}}\left[^{\circ}\right]$ & $\sigma[\mathrm{dB}]$ \\
\hline \hline True & $\mathbf{1 0 0}$ & 2.3 & $\mathbf{6 5}$ & $\mathbf{7}$ & 4 \\
\hline Est. $(m=1 e 3)$ & 100.25 & 2.35 & 64.83 & 7.09 & 3.97 \\
\hline Est. $(m=2 e 3)$ & 99.87 & 2.36 & 64.99 & 6.91 & 4.06 \\
\hline Est. $(m=5 e 4)$ & 100.05 & 2.32 & 64.98 & 7.02 & 4.01 \\
\hline
\end{tabular}

Fig. 4.2 for the method using the approximate boundary conditions. It can be observed that the estimated path loss slope is in good agreement with the true slope. Note, that the RSS measurements still contain the contribution from the antenna gain and thus the true and estimated slope do not follow the trend of the RSS values. For comparison purposes, we have also included the path loss slope when the estimator is only estimating the path loss parameters, i.e. $G_{\text {ant }}=0$ in the estimator model. In this case, we have a model mismatch and the path loss slope follows the trend of the RSS measurements, as expected.

For the model mismatch case, we also included the estimation results for different number of RSS measurements taken from the approximate boundary conditions, which are shown in Table 4.4. It is now also observed that the error standard deviation is much larger, which is due to the compensation of unmodeled antenna gain variations. The benefits of adopting a joint antenna and propagation model in comparison to only a propagation model is this evident. 


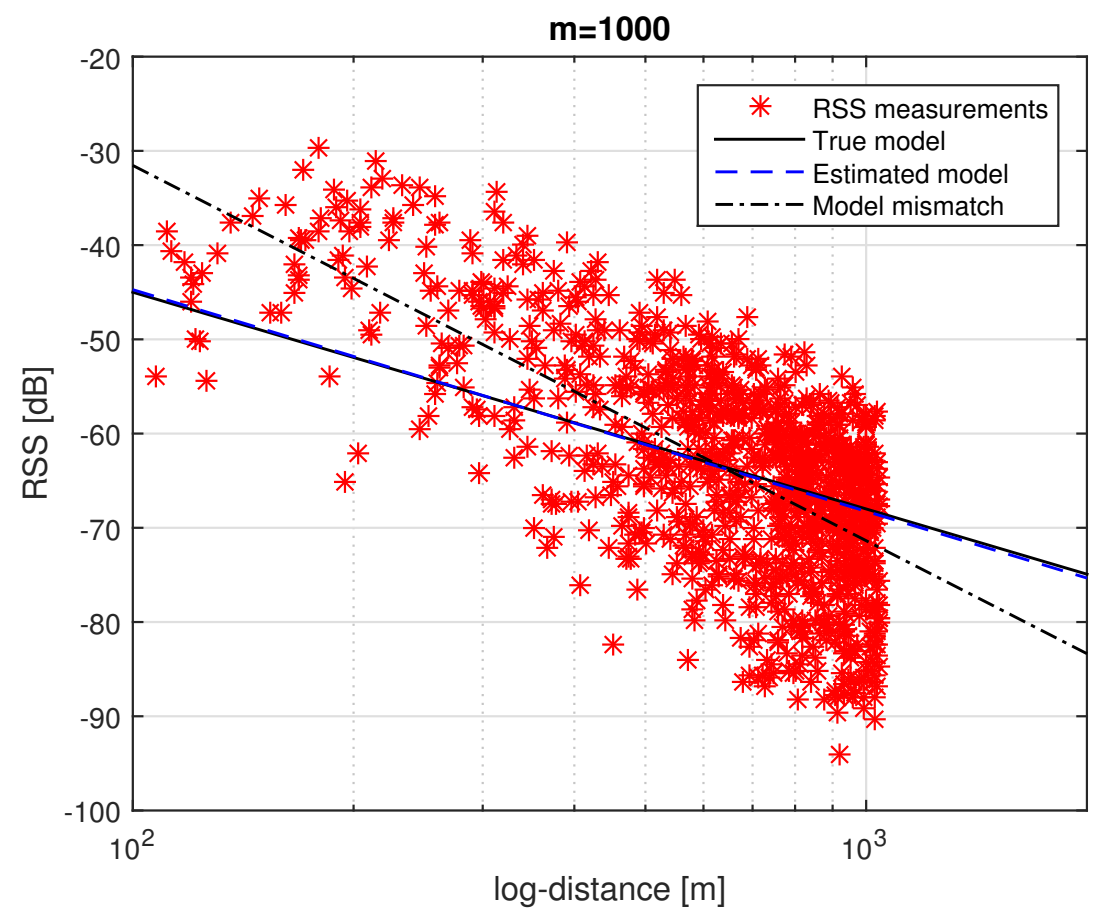

Figure 4.2: Path loss estimation results for simulated data using approximate boundary conditions and $m=1000$ [47, C2015 IEEE].

\subsection{Real Data Experiments}

The proposed joint antenna and propagation model described in the previous section is also verified with real-field measurements. The measurements are collected in a rural/suburban area, with an Android app for logging A-GPS and RSS [39]. The smartphone was configured to camp on a 3GPP LTE/E-UTRAN network, which means that the logging reflects Reference Signal Received Power.

In order to identify the parameters accurately, a direct LOS between the MS and BS is preferable. One base station is chosen in an area where buildings and similar structures that can obscure a direct LOS are rather sparse. The selected base station is equipped with antennas mounted on a mast which is $30 \mathrm{~m}$ high. The BS serves three cells each covering 120 degrees, and one of the cells (with an antenna boresight direction $\phi_{0}=-30^{\circ}$ ) is used for evaluation purposes. The antenna associated to the cell has a halfpower beamwidth of 60 degrees. However, note that this is the beamwidth observed near the antenna. Signal scattering will spread the signals, effectively creating an antenna that is perceived to be wider by a distant observer. It is therefore expected that the estimated horizontal halfpower beamwidth is slightly wider than the nominal beamwidth of 60 degrees, and the vertical halfpower beamwidth wider than the nominal bandwidth of 7 
Table 4.4: Estimation results for model mismatch and approximate boundary conditions [47, C2015 IEEE].

\begin{tabular}{|l||c|c|c|}
\hline Parameter & $A[\mathrm{~dB}]$ & $B[\mathrm{~dB}]$ & $\sigma[\mathrm{dB}]$ \\
\hline \hline True & $\mathbf{1 0 0}$ & 2.3 & 4 \\
\hline Est. $(m=1 e 3)$ & 103.37 & 3.98 & 8.40 \\
\hline Est. $(m=2 e 3)$ & 103.21 & 3.84 & 8.55 \\
\hline Est. $(m=5 e 4)$ & 103.39 & 4.01 & 8.68 \\
\hline
\end{tabular}

degrees.

The measurements are collected over several trajectories around the site as depicted in Fig. 4.3. In the same figure, the RSS values are also presented with colors.

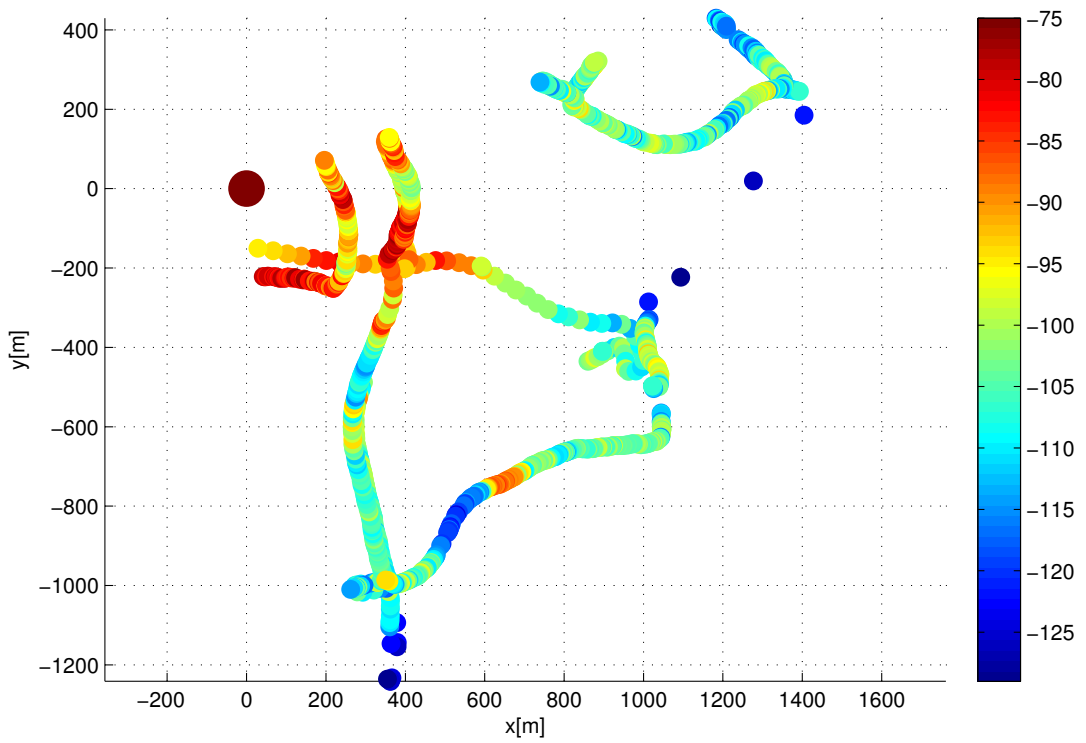

Figure 4.3: The RSS values collected along different trajectories around the antenna. Antenna location is shown by the big circle [47, C2015 IEEE].

Measurements were selected that are assumed to be in the horizontal main lobe of the antenna, with boundary conditions $\left|\phi-\phi_{0}\right| \leq 50^{\circ}$. The region is quite flat and the trajectories are not in close vicinity of the antenna. Therefore there is not much variety in vertical angles. The contribution from the vertical antenna gain is expected to be smaller than $0.1 \mathrm{~dB}$ under the assumption of nominal value of the parameters. Hence its effect is neglected for the scenario, and the vertical antenna gain component is omitted from the joint model. The estimated parameters under these assumptions are given in Table 4.5. As expected, the estimated effective horizontal antenna halfpower beamwidth $\phi_{\mathrm{h}}$ is wider than the nominal 
beamwidth due to scattering. Furthermore, the residual standard deviation of 7 $\mathrm{dB}$ is in the expected range of $6-10 \mathrm{~dB}[34]$.

Table 4.5: Estimation results for real data experiments using only horizontal antenna gain pattern [47, O2015 IEEE].

\begin{tabular}{|l||c|c|c|c|}
\hline Parameter & $A[\mathrm{~dB}]$ & $B[\mathrm{~dB}]$ & $\phi_{\mathrm{h}}\left[^{\circ}\right]$ & $\sigma[\mathrm{dB}]$ \\
\hline \hline Est. $(m=1252)$ & 152.6 & 2.9675 & 79.5823 & 7.0265 \\
\hline
\end{tabular}

Fig. 4.4 illustrates the fit of the propagation model component, which indicates a good fit. These brief evaluations indicate the relevance and benefits of joint antenna and propagation model parameter estimation.

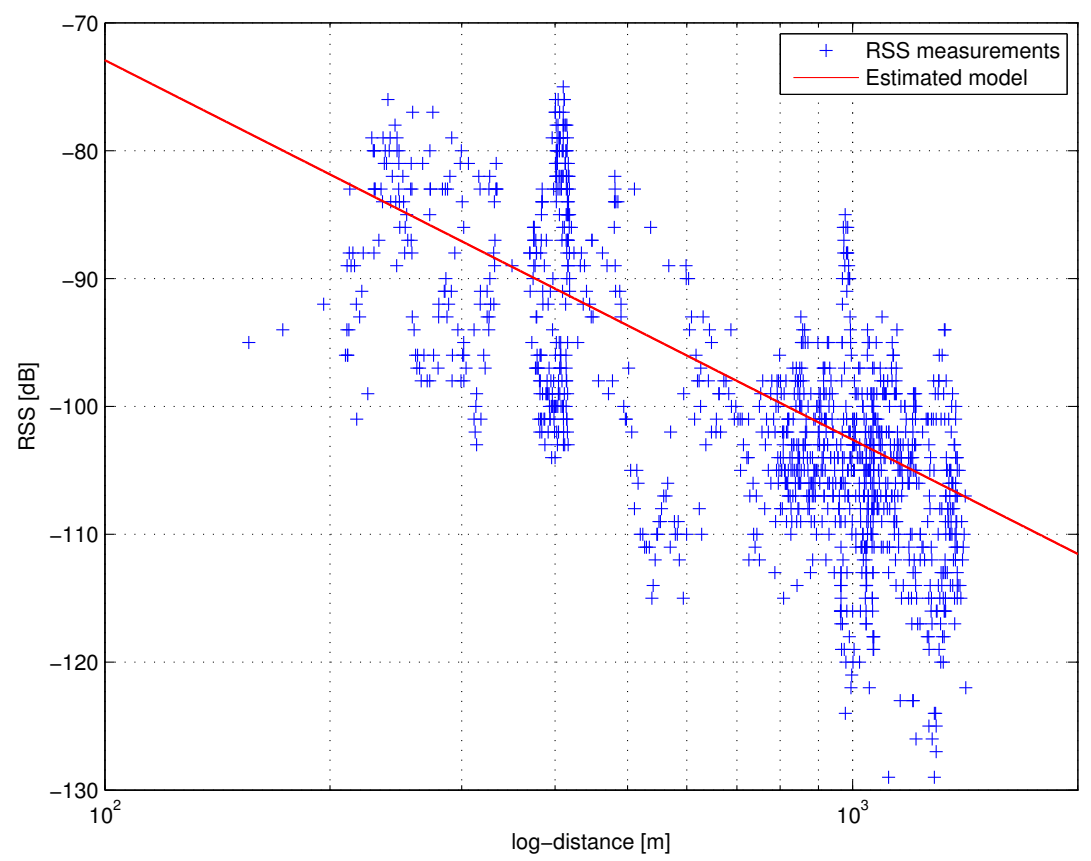

Figure 4.4: RSS measurements together with estimated path loss model [47, C2015 IEEE]. 


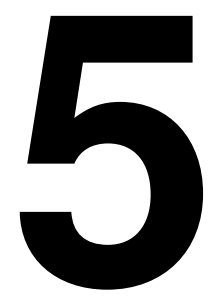

\section{Gait parameter Estimation Learning}

This chapter, extends the general state estimation problem introduced in Chapters 2 and 3 to solve a personal navigation problem. In this framework, the state of the system is further extended by IMU sensors and a step length parameter. An earlier version of this application, some part of its background theory and also parts of the proposal solution were previously published by the author in (FUSION2016) [48, (O2016 IEEE]. In this chapter we will presents the results given by the approaches proposed by author in [48]. Additionally, an extended version of these approaches are presented in detail in this chapter.

We propose an improved pedestrian dead reckoning (PDR) algorithm that learns gait parameters in time intervals when GPS is available, for instance from GPS or an indoor positioning system (IPS). Then, the learned gait parameters are used for state estimation when no position measurement is available. Internal gait parameters in the PDR algorithm, such as the step length and the step detection threshold are estimated by a novel filtering approach. The proposed approach is based on a multi-rate Kalman filter bank that estimates the gait parameters when position measurements are available to enhance the performance of the PDR in time intervals when such measurements do not exist. One motivating example is the scenario in which navigation is requested when the user's path contains both outdoor and indoor intervals, the GPS measurement is not always available. The effectiveness of the new approach is investigated on several simulated data as well as real world experiments.

We start by introducing PDR models which are extended versions of the model presented in [48] in Section 5.1. The step length has been used in these proposed methods as a state, therefore we need to detect the steps in advance. Section 5.2 introduces the step detection algorithm and step length estimation methods. The filtering solution of the considered application is explained in Section 5.3. Section 5.4 discusses the dead reckoning improvements by learning the gait param- 
eters on a number of real-world experiments.

\subsection{Pedestrian Odometry}

In pedestrian navigation systems, the double-integrating strap-down method suffers from an error in the position estimate. This error propagates and increases quadratically with time [88]. Integrating gait parameters could be a solution to this issue. The gait parameters can be utilized in PDR algorithms to improve the performance.

Traditionally, PDR algorithms detect gait parameters, such as number of detected steps and step length, in order to determine the travelled distance. This approach is applied for navigation applications in many studies, e.g [71, 72]. The basic longitudinal model is given by

$$
d_{s+1}=d_{s}+L+n_{s} \text {, }
$$

where $d_{s}$ is the total walked distance, $s$ is the step index, $L$ is the step length and $n_{s}$ is noise. Estimating a pedestrian's step length and determining a suitable threshold for step detection from IMU accelerometer measurements need to be treated appropriately as is discussed in Section 5.2. However, we first introduce the extended versions of the pedestrian odometric model in the following subsections.

\subsubsection{Extended Pedestrian Odometric Model}

States of the extended pedestrian odometric model contain extra components defined by the dimension of the system. One dimensional models, known as longitudinal models, add only the travelled distance $d$ while in two dimensional, horizontal, models the step length, angular rate, and bias are also added to the conventional pedestrian odometric model.

\section{Longitudinal model}

A longitudinal multi-rate model is proposed that is updated each time a step is detected or a GPS position estimate becomes available. The underlying motion model is the standard constant velocity (CV) model extended with step length. This model has a state vector $\mathbf{x}=[d, v, L]^{T}$ with travelled distance $d$, velocity $v$ and step length $L$. This model was introduced in [48].

The dynamic model from one event $t_{k}$ (either GPS position observed or step detected) to the next event at $t_{k+1}$ is given by

$$
\mathbf{x}_{t_{k+1}}=\mathbf{F}\left(t_{k+1}-t_{k}\right) x_{t_{k}}+\mathbf{G}\left(t_{k+1}-t_{k}\right) \mathbf{w}_{t_{k+1}},
$$

where $\mathbf{w}_{t_{k+1}}$ denotes process noise

$$
\mathbf{w}_{t_{k+1}} \sim \mathcal{N}(0, \mathbf{Q}), \quad \mathbf{Q}=\left[\begin{array}{cc}
\sigma_{a}^{2} & 0 \\
0 & \sigma_{L}^{2}
\end{array}\right] .
$$


The matrices $F$ and $G$ are given by

$$
\mathbf{F}(T)=\left[\begin{array}{lll}
1 & T & 0 \\
0 & 1 & 0 \\
0 & 0 & 1
\end{array}\right], \quad \mathbf{G}(T)=\left[\begin{array}{cc}
\frac{T^{2}}{2} & 0 \\
T & 0 \\
0 & 1
\end{array}\right] .
$$

where $T$ is the sampling time. In this model, the measurement data is asynchronous, i.e. the time elapsed between two filter updates will depend on which measurement (step detected or GPS position observed) is currently processed. The general form of a measurement model is given by

$$
y_{k}=\mathbf{H}_{k} \mathbf{x}_{k}+e_{k}
$$

where $\mathbf{H}_{k}$ is a mapping matrix and $e_{k}$ denotes measurement noise. In case a GPS position estimate is available, it is converted to a corresponding velocity as follows

$$
v_{t_{l}}=\frac{\left\|\hat{\mathbf{p}}_{t_{l}}-\hat{\mathbf{p}}_{t_{l-1}}\right\|}{t_{l}-t_{l-1}}
$$

where $\|\cdot\|$ denotes the $L_{2}$ norm and $\hat{\mathbf{p}}_{t_{l}}$ and $\hat{\mathbf{p}}_{t_{l-1}}$ denote the GPS position estimates available from the current time instance $t_{l}$ and the previous time stamp $t_{l-1}$. It is then possible to express the model for the GPS measurement as follows

$$
y_{t_{l}}^{\mathrm{GPS}}=\mathbf{H}_{t_{l}} \mathbf{x}_{t_{l}}+e_{t_{l}}^{\mathrm{GPS}},
$$

where $e_{t_{l}}^{\mathrm{GPS}}$ is noise assumed to be zero-mean Gaussian distributed with variance $\sigma_{\mathrm{v}, \mathrm{GPS}}^{2}$, and $H_{t_{l}}=[0,1,0]$. Since there are no direct observations for step detection available, we define an artificial measurement that is used whenever a step is detected (the step detection algorithm is given in Section. 5.2). The step detection measurement model is given by

$$
y_{t_{s}}^{\text {step }}=\mathbf{H}_{t_{s}} \mathbf{x}_{t_{s}}+e_{t_{s}}^{\text {step }}
$$

where $H_{t_{s}}=\left[0, \Delta t_{s},-1\right], \Delta t_{s}=t_{s}-t_{s-1}$ is the time difference between two consecutive step detections regardless of how many GPS measurements are detected in between, and $e_{t_{s}}^{\text {step }}$ is noise assumed to be zero-mean Gaussian distributed with variance $\sigma_{\text {step. }}^{2}$.

This longitudinal model is linear in the state, and the solution for this problem fits the linear Kalman filter.

\section{Horizontal model}

Here a 2-D multi-rate horizontal model is proposed. It is based on a Coordinated Turn (СT) model with polar velocity extended with step length. A preliminarily version of this model has been introduced in [48].

The nonlinear CT allows for a varying turn rate and also a varying target speed. The proposed model has a state vector $\mathbf{x}$ with seven components: position $\mathrm{x}$, position $\mathrm{y}$, polar velocity $v$, heading $\psi$, heading rate $\dot{\psi}$, yaw rate $\dot{\omega}$ bias $b$, and step 
length $L$. The resulting differential equation for the state is given by

$$
\dot{\mathbf{x}}=\left[\begin{array}{c}
\dot{x} \\
\dot{y} \\
\dot{v} \\
\dot{\psi} \\
\dot{\omega} \\
\dot{b} \\
\dot{L}
\end{array}\right]=\left[\begin{array}{c}
v \cos (\psi) \\
v \sin (\psi) \\
a \\
\omega \\
\alpha \\
0 \\
0
\end{array}\right]
$$

where $a$ is linear acceleration and $\alpha$ is turn rate. The differential equations require discretization in order to apply discrete-time filtering techniques. The result has the form

$$
\mathbf{x}_{t_{k+1}}=\mathbf{f}\left(\mathbf{x}_{t_{k}}, t_{k+1}-t_{k}\right)+\mathbf{g}\left(\mathbf{x}_{t_{k}}, t_{k+1}-t_{k}\right) \mathbf{w}_{t_{k+1}},
$$

where $\mathbf{w}_{t_{k+1}}$ is assumed to be uncorrelated zero-mean Gaussian noise with covariance matrix $\mathbf{Q}=\operatorname{diag}\left(\left[\sigma_{a}^{2}, \sigma_{\alpha}^{2}, \sigma_{L}^{2}\right]\right)$. The discretization of (5.9) yields

$$
\mathbf{f}(\mathbf{x}, T)=\left[\begin{array}{c}
x+\frac{2 v}{\omega} \sin \left(\frac{\omega T}{2}\right) \cos \left(\psi+\frac{\omega T}{2}\right) \\
y+\frac{2 v}{\omega} \sin \left(\frac{\omega T}{2}\right) \sin \left(\psi+\frac{\omega T}{2}\right) \\
v \\
\psi+\omega T \\
\omega \\
b \\
L
\end{array}\right],
$$

where we have assumed $a$ and $\alpha$ to be zero. The zero-order-hold discretization [33] is applied in order to find $g(\mathbf{x}, T)$ which is given by

$$
\mathbf{g}(\mathbf{x}, T)=\left[\begin{array}{cccc}
\frac{T^{2}}{2} \cos (\psi) & 0 & 0 & 0 \\
\frac{T^{2}}{2} \sin (\psi) & 0 & 0 & 0 \\
T & 0 & 0 & 0 \\
0 & \frac{T^{2}}{2} & 0 & 0 \\
0 & T & 0 & 0 \\
0 & 0 & 1 & 0 \\
0 & 0 & 0 & 1
\end{array}\right]
$$

For the horizontal model it is assumed that 2-D GPS position estimates, step detection as well as turn rates from gyroscopes are available as measurements. The model for the GPS position estimates is given by

$$
\mathbf{y}_{t_{l}}^{\mathrm{GPS}}=\left[\begin{array}{ccccccc}
1 & 0 & 0 & 0 & 0 & 0 & 0 \\
0 & 1 & 0 & 0 & 0 & 0 & 0
\end{array}\right] \mathbf{x}_{t_{l}}+\mathbf{e}_{t_{l}}^{\mathrm{GPS}},
$$

where $\mathbf{e}_{t_{l}}^{\text {GPS }}$ is noise on the GPS position estimates, which is assumed to be zeromean Gaussian distributed with covariance matrix $\mathbf{R}^{\mathrm{GPS}}=\operatorname{diag}\left(\left[\sigma_{\mathrm{p}, \mathrm{GPS}}^{2}, \sigma_{\mathrm{p}, \mathrm{GPS}}^{2}\right]\right)$. 
The measurement model for the step detection is analogous to the longitudinal model (5.8) and is given by

$$
y_{t_{s}}^{\text {step }}=\left[\begin{array}{lllllll}
0 & 0 & \Delta t_{s} & 0 & 0 & 0 & -1
\end{array}\right] \mathbf{x}_{t_{s}}+e_{t_{s}}^{\text {step }},
$$

The gyroscope measurement model is given in 3.2. For convenience, we restate it in special form for our case as

$$
y_{t_{g}}^{\text {gyro }}=\omega_{z, t_{g}}+b_{t_{g}}^{\text {gyro }}+e_{t_{g}}^{\text {gyro }}
$$

where $\omega_{z, t_{g}}$ is the yaw rate which is the gyroscope measurement in the horizontal plane, $b$ gyro denotes gyroscope bias, and $t_{g}$ denotes the time instance when gyroscope measurements are available, and $e_{t_{g}}^{\text {gyro }}$ denotes noise assumed to be zeromean Gaussian with variance $\sigma_{\text {gyro }}^{2}$. The gyroscope measurement model with bias given by

$$
y_{t_{g}}^{\text {gyro }}=\left[\begin{array}{lllllll}
0 & 0 & 0 & 0 & 1 & 1 & 0
\end{array}\right] \mathbf{x}_{t_{g}}+e_{t_{g}}^{\text {gyro }} .
$$

In contrast to the longitudinal model, the horizontal model is nonlinear in the states due to the nonlinear process model. Hence, nonlinear filters have to be used for state estimation, and we will make use of the Extended Kalman filter in our proposed PDR solution.

\subsection{Step detection}

As mentioned earlier, step detection is one of the challenging issues of using PDR algorithm for navigating pedestrians. Here, we overview the recent research work that have been done concerning this problem and then propose an algorithm for estimating the gait parameter.

Most used signals for step detection purposes are those corresponding to acceleration and angular rate. Depending on the application at hand two detection algorithm are used. In scenarios with body-mounted devices, static detection is performed while in case of hand-held devices zero-cross detection and peak detection are applied. Mistaken detections in these methods are due to multiple peak values per step. To address this false detection problem, $[58,83,75,76,71]$ look at the norm of the total acceleration rather than looking only at the vertical acceleration component.

Another possible source of error in step detection algorithms is the orientation of the sensor that is used for measuring signals. For instance, IMUs embedded in portable devices. To avoid disturbances caused by this arbitrary orientation on the vertical signal component, [45] projects acceleration in local coordinate system to a global one. In this way, it is possible to resolve issues occurred due to the placement of the device.

Accurate estimation of the number of steps from the peaks of accelerometer signal could be possible by applying a filter. That is, by means of a low pass filter, one can modify the output waveform of the tri-axial accelerometer and 
then count the number of peaks. In this regard, [58] takes a window length of size 10. Applying a band-pass filter rather than a low-pass is also studied in the literature. [94] applies such a filter with a low and high cut-off value. Then, by comparing steps and the threshold value, they estimate the number of steps.

Pre-processing the raw accelerometer signal is investigated by [45]. After the pre-process phase a high-pass filter is applied to the acceleration on $z$-axis in global coordinates to remove effects of Earth's gravity. They finally perform a lowpass filtering procedure to reduce random noise. [40] on the other hand, apply a fast Fourier transform to smooth the raw acceleration signal for step detection.

In case of hand-held devices, extra attention is needed because it is often swing in the user's hand. The swinging pattern generates incorrect peaks that need to be addressed. [58] introduces two thresholds $\tau$ and $\sigma$ for time and peaks to remove the errors involved in step counting. Depending on the sample frequency and data analyses and test results, they adjust values of these thresholds. Taking advantages of the motion pattern is another proposal solution [76]. They apply different peak thresholds in different step detection algorithms based on the motion pattern. In [71], it is shown that it is possible to use a motion recognition first and then use accelerometer and gyroscope signal in order to detect the steps.

\subsubsection{Step Detection Algorithm}

As it is considered in many research works, the three-axis accelerometer signal contains all the data required to detect the occurrence of a step. The vertical axis, relative to the ground, contains all information corresponding to the step specific peak. However, the orientation of the sensor may cause some disturbances on the vertical signal component. For instance, in case the IMU is embedded in handheld devices such as smartphones. In order to avoid disturbances, the norm of the total acceleration is used instead of looking only at the vertical acceleration component. Algorithm 1 illustrates the step detection algorithm. In this algorithm, the variables $a_{x}, a_{y}$ and $a_{z}$ denote the tri-axial accelerometer components. In order to improve the quality of the signal, a Butterworth band-pass filter with a proper cut-off frequency is applied to attenuate all frequencies outside the bandpass.

Defining a suitable threshold $h$ in order to correctly detect steps is one difficulty of PDR algorithms. The threshold needs to be adjusted for different people with different characteristics. In order to make our point clear, we have designed an experiment in which three people with different attributes (height and weight) and walking behaviors have participated. All of them walked along the same rectangular trajectory. In order to have a better classification per person, they were asked to walk in a slow pace and count their steps, so that these can be used as the ground truth. As Fig. 5.1 shows the threshold for each user is unique. Furthermore, choosing a too large threshold will underestimate the number of detected steps. For instance, the number of detected steps for user 2 drops rapidly for thresholds larger than $1 \mathrm{~m} / \mathrm{s}^{2}$. A change of the user's speed is another aspect that may affect the threshold. Fig. 5.2 illustrates the step detection performance for 
Algorithm 1 Step Detection [48, O2016 IEEE].

- Input: Three-axis Accelerometer Signal

1. Compute the norm of the accelerometer signal:

$$
a=\sqrt{a_{x}^{2}+a_{y}^{2}+a_{z}^{2}} .
$$

2. Band-pass filter the resulting signal using a fourth-order Butterworth filter with cut-off frequency $[0.2,2.75] \mathrm{Hz}$.

3. If the filtered signal exceeds a defined threshold $h$, a step is considered detected.

4. Among all sets of accelerations that are larger than the threshold, before the signal again drops below the threshold, the one with the highest value is selected as the step.

- Output: Step detections

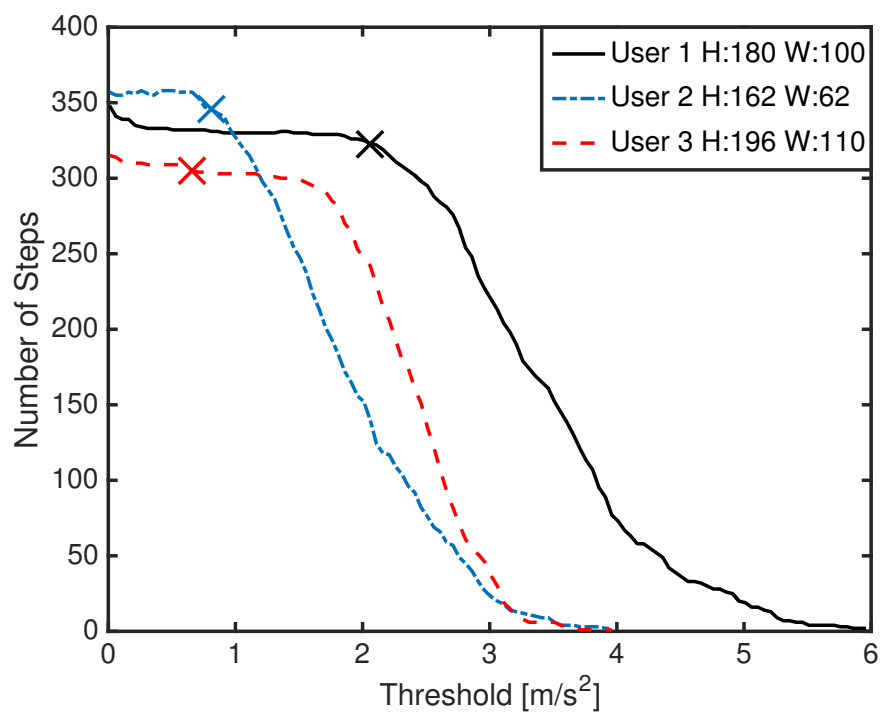

Figure 5.1: Number of steps detected vs. step detection threshold $h$ for users with different height $(H)$ and weight $(W)$. The crosses indicate true number of steps [48, O2016 IEEE].

different paces of a single user. We observe that different speeds yield different step detection curves, and choosing the right threshold becomes important. It is worth noting that in all of our experiments the user is walking all the time. 


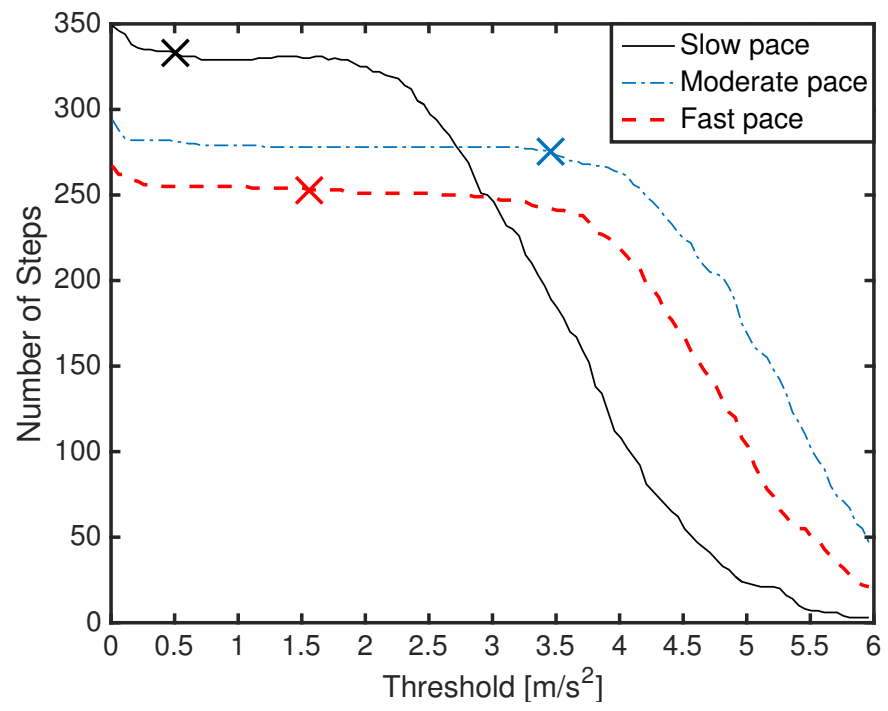

Figure 5.2: Number of steps detected vs. step detection threshold $h$ for two different users with different walking modes. The crosses indicate true number of steps [48, C2016 IEEE].

This means, that below a certain threshold the step detection curves become (relatively) flat. Hence, one could argue that a properly chosen (small enough) fixed threshold might be sufficient to obtain good step detection performance. However, in reality the user is not walking all the time. The user motion will rather be interrupted by shorter (or longer) periods of stand still, which yields step detection curves that are no longer flat and would result in a significant increase of false detections if the threshold is chosen too small. The effect of different motion modes is illustrated in Fig. 5.3 where two users were asked to walk with different modes and as in the previous case, the ground truth is their counts of taken steps. The step detection curves are no longer flat and a too small threshold results in too many false detections.

\subsubsection{Step Length Estimation}

Lately, much research has been conducted on step length estimation $[45,58,83$, 40]. One method for estimating the step length is to consider the relations between output waveform of the tri-axial accelerometer and step length that is known as the empirical formula in this method, the step length is computed by using the difference between maximum and minimum output waveform of a triaxial accelerometer in each step respectively. Then, a calibration factor based on the ratio of the real and estimated reference trajectory distances must be applied.

The estimation approach using the compositional algorithm of empirical formula and back propagation (BP) neural network is studied in $[58,83]$. In $\mathrm{BP}$ neu- 


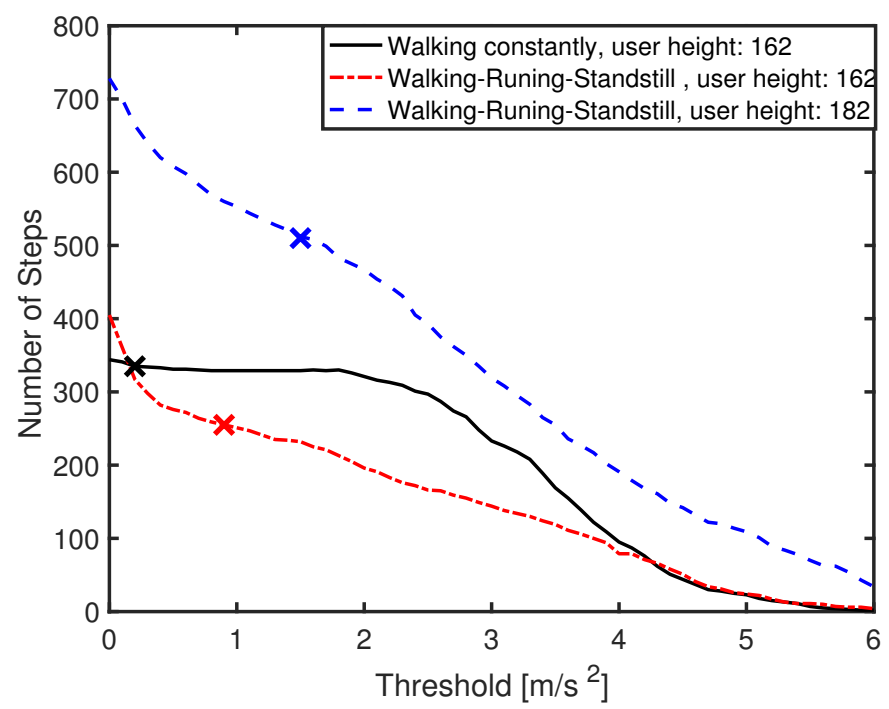

Figure 5.3: Number of steps detected vs. step detection threshold $h$ for a single user with different walking paces. The crosses indicate true number of steps.

ral network, the accuracy of estimating the step lengths cannot be guaranteed since the model estimate of the step length is not accurate.

In [40], the calibration factor of the empirical formula is selected adaptively. That is, instead of using a constant factor for all steps, a polynomial function of the average step velocity is used for calibration factor derivation. The degree of the polynomial is then given by an $N$-fold cross-validation being applied on the dataset.

Linear formula is another common method for step length estimation. In case of walking or running pedestrians, [94] applies the linear formula to estimate the step length. Pre-initializd parameters required in this method are obtained by analyzing a large amount of experiment data.

Depending on the motion pattern, [94] uses different formulas to estimate the step length. Going up or down the stairs is studied there. The horizontal length of the stair and the threshold determined by the step speed are used for step length estimation.

A short time Fourier transform (STFT) based method is introduced in [71]. Independently of the step detection process, they use STFT to extract the frequency content of the device's signal. Based on this detected frequency and linear relationship between frequency and step length the step length is estimated.

In our proposed algorithm, the step length is considered as a state in our model and will be estimated in both online and offline ways using multi-rate filtering and filter bank. It means that the step length estimation process does not 
require any pre-initialized parameters and also it is compatible with the pedestrian speeds which may vary due to changing the walking pattern.

\subsection{Filter Bank}

There are applications where the system needs to have multiple operational models. The estimator for such systems can then consist of a bank of parallel filters each applied to one mode of the system. The generic model (2.14) then becomes

$$
\begin{aligned}
\mathbf{x}_{k+1} & =\mathbf{f}_{k}\left(\mathbf{x}_{k}, \mathbf{w}_{k}, \boldsymbol{\delta}_{k}\right), \\
\mathbf{y}_{k} & =\mathbf{h}_{k}\left(\mathbf{x}_{k}, \mathbf{e}_{k}, \boldsymbol{\delta}_{k}\right),
\end{aligned}
$$

where $\delta_{k}$ denotes the mode at time instant $k$. The filter bank treats different filters individually. Assuming that each filter is the correct one, the estimator computes the likelihood of each filter. The state estimates obtained from all the filters are then blended and weighted by their likelihood. One challenge in constructing filter banks is to avoid exponential growth of the number of parallel filters. For more information consult [37].

To keep the number of filters in a feasible range, different methods exists. Interacting multiple model (IMM), Generalized peseudo-Bayesian filter [12], and the adaptive forgetting through multiple models [6] are major available methods.

The filter bank that is used in this work is tailored to our case and is slightly different from the standard form of multiple model adaptive estimators. The number of the parallel filters is fixed and the true threshold is considered as an unknown parameter and parallel filters run with different hypotheses. Subsequently, all mode probabilities need to be evaluated to find the best fit threshold.

The mode probabilities are estimated by the filter bank in both offline and online fashions. In the offline case, the threshold has been estimated by minimizing a likelihood cost function where the proposed approach processes batches of real data for each experiment. The result of this work have been presented in [48]. In the online case, the proposed approach processes the data sequentially and all mode probabilities are merged to find the best fit threshold at each time. IMM filter is the method that is used for merging the mode probabilities in our study. The transition probability matrix (TPM), $\pi$, in IMM filter is assumed to be known. In the rest of this section offline and online filter bank estimators are described in more details.

\subsubsection{Offline Kalman Filter Bank}

The block diagram of the proposed PDR algorithm that learns gait parameters is shown in Fig. 5.4. The algorithm basically consists of four blocks. In the step detection filter block, $N$ different thresholds are applied to a bank of step detectors, whose inputs are the accelerometer signals. The detected steps, as the output of this filter, are passed to the Kalman filter bank block consisting of $N$ multi-rate (Extended) Kalman Filters, that process the step detections, GPS position estimates (and gyroscope measurements). The results of this filter bank are 
then passed to the state estimation and threshold selection block. The threshold selection block is processing $N$ threshold dependent innovation vectors $\varepsilon_{k}(h)$ and corresponding innovation covariances $\mathbf{S}_{k}(h)$ to obtain a threshold estimate $\hat{h}$ for the step detector based on minimizing some likelihood cost function. In the state estimation block, the $N$ estimated states together with the error covariances and other filter parameters are inputs to a mixing stage that produces the final filter output consisting of a state vector estimate $\hat{\mathbf{x}}$ and corresponding error covariance $\mathbf{P}$ [33].

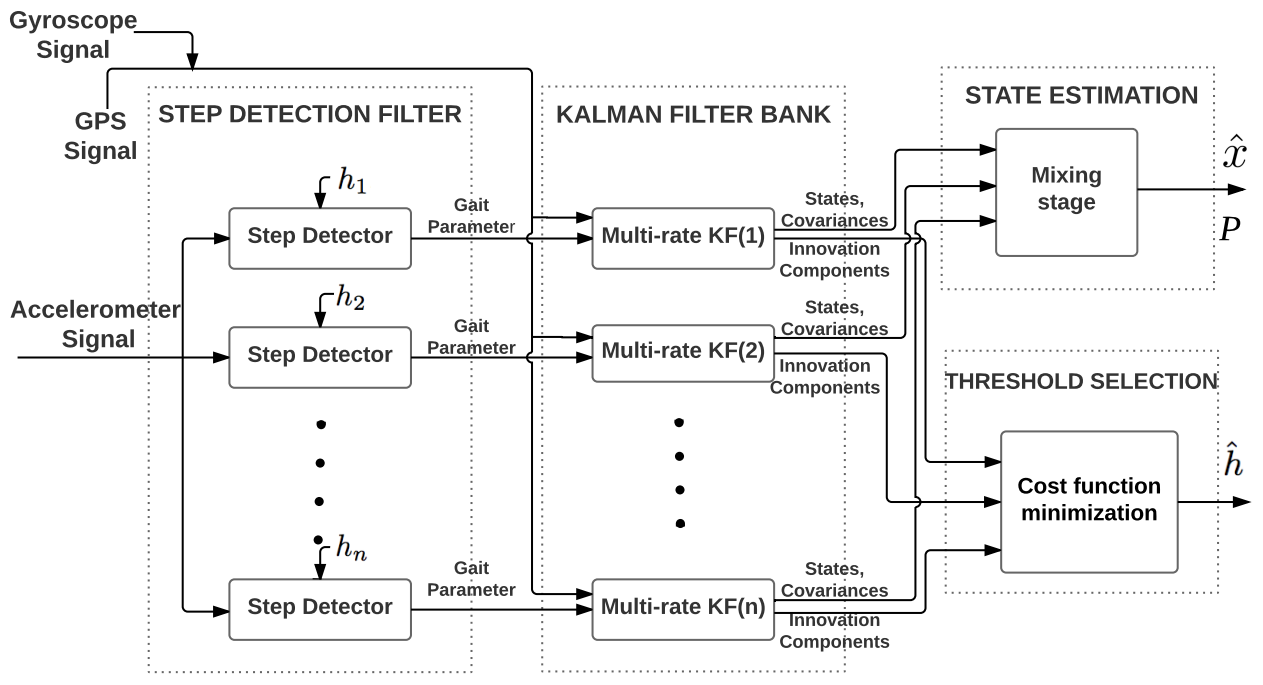

Figure 5.4: Block diagram of PDR algorithm with gait parameter learning [48, C2016 IEEE].

As mentioned earlier, the majority of blocks have been only tested by processing batches of real data for each experiment. In particular, all blocks except the state estimation block, which was not tested, were each processed offline using batches of data. This means that the step detection filter processed all data at once. The output of this filter, a batch of detected steps, was then fed into the Kalman filter bank to output a batch of innovations and covariances. The threshold estimation block has been adapted to process batches of data. More specifically, the threshold estimate $\hat{h}$ for each experiment is obtained by minimizing the following likelihood cost function

$$
\hat{h}=\arg \min _{h} \frac{\sum_{k=1}^{N} \varepsilon_{k}^{T}(h) \mathbf{S}_{k}^{-1}(h) \varepsilon_{k}(h)+\log \mathbf{S}_{k}(h)}{N},
$$

where $N$ is the length of the batch. A small $h$, gives many false detected steps resulting in large innovations. Conversely, a large $h$ results in that many steps being missed, yielding large innovations again. While the proposed analysis is not fully sequential, it gives a very good insight on the potential of the proposed approach for PDR and gait parameter learning. 


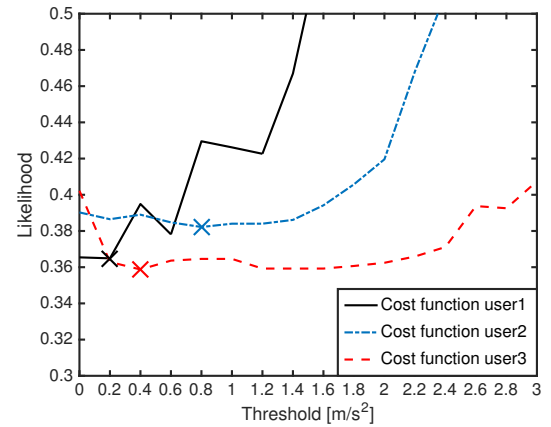

(a) Three different users with different characteristics.

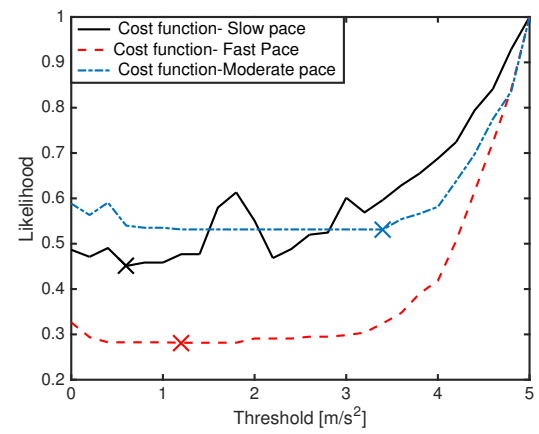

(b) A single user with three different walking paces.

Figure 5.5: Likelihood cost function vs. step detection threshold $h$. The crosses indicate the estimated threshold $\hat{h}$ where the likelihood cost function is minimized [48, C2016 IEEE].

Fig. 5.5a and 5.5b present results for the threshold selection block. Here, the (batch) of measurement data corresponding to three different users all walking slowly but with different physical characteristics and the data corresponding to the single user with different walking paces have been processed. The data batches used are the same that have generated Fig. 5.1 and Fig. 5.2. It can be observed that all cost functions are different and yield different estimated $\hat{h}$ values when the likelihood cost function is minimized. In Table 5.1, the estimated thresholds from minimizing the likelihood cost function are compared to the "true" thresholds obtained from counting the total number of steps during the experiments. It can be observed that the estimated thresholds $\hat{h}$ are in good agreement with the true ones, and hence the criterion given in (5.18) seems to be a good indicator to choose the step detection threshold.

Table 5.1: Estimated step detection threshold $\hat{h}$ versus true threshold [48, (C)2016 IEEE].

\begin{tabular}{|c|c|c|}
\hline & $\begin{array}{c}\text { True Threshold } \\
{\left[\mathrm{m} / \mathrm{s}^{2}\right]}\end{array}$ & $\begin{array}{c}\text { Estimated threshold } \\
{\left[\mathrm{m} / \mathrm{s}^{2}\right]}\end{array}$ \\
\hline User 1 & 2.06 & 2.0 \\
\hline User 2 & 0.81 & 0.8 \\
\hline User 3 & 0.61 & 0.4 \\
\hline Slow pace & 0.51 & 0.6 \\
\hline Moderate pace & 3.46 & 3.4 \\
\hline Fast pace & 1.56 & 1.4 \\
\hline
\end{tabular}




\subsubsection{Online Kalman Filter Bank}

This section proposes an online PDR algorithm featuring gait parameters learning using IMM. In order to simplify the procedure first a single step of the IMM filter is presented as an algorithm and then the whole algorithm is presented in a block diagram and each block is described in detail.

\section{IMM Filter}

A single step of the IMM filter is given here. Let $\left\{\hat{\mathbf{x}}_{k \mid k}^{j}, \mathbf{P}_{k \mid k}^{j}, \mu_{k}^{j}\right\}_{j=1}^{N}$ denote the previous sufficient statistics. Then, a single step of the IMM algorithm to obtain current sufficient statistics $\left\{\hat{\mathbf{x}}_{k+1 \mid k+1}^{i}, \mathbf{P}_{k+1 \mid k+1}^{i}, \mu_{k+1}^{i}\right\}_{i=1}^{N}$ is described in the sequel in Algorithm $2[9,10]$.

\section{Online Kalman Filter Bank Using IMM}

The block diagram of the proposed online PDR algorithm featuring gait parameters learning is shown in Fig. 5.6. The block diagram consists of six blocks. The "mixing probability calculation" block processes $N$ mode probabilities $\mu_{k}^{i}$ to obtain mixing probability $\mu_{k}^{j i}$ presented in the "Mixing" step in Algorithm 2. $\pi$ in the mixing probability equation is the TPM, and as mentioned earlier, it is set to an identity matrix where no switching are assumed between the modes. The "Mixing block" processes $N$ updated estimates $\hat{\mathbf{x}}_{k \mid k}^{j}$, covariances $\mathbf{P}_{k \mid k}^{j}$, and mixing probabilities $\mu^{j i}$, which is the output of the mixing probability calculation block, to obtain mixed estimates $\hat{\mathbf{x}}_{k}^{0 i}$ and covariances $\mathbf{P}_{k}^{0 i}$ presented in the "Mixing" step in Algorithm 2.

In the "step detection filte" block, $N$ different thresholds are applied to a bank of step detectors, whose inputs are the accelerometer signals. The detected steps, as the output of this filter, and mixed estimates $\mathbf{x}_{k}^{0 i}$ and covariances $\mathbf{P}_{k}^{0 i}$, as the output of the mixing block, are passed to the Kalman filter bank block which is equipped with $N$ multi-rate (extended) Kalman filters.

The KF bank block processes the step detection, mixed estimates $\mathbf{x}_{k}^{0 i}$, covariances $\mathbf{P}_{k}^{0 i}$ and GPS position estimates (and gyroscope measurements). The updated estimate $\hat{\mathbf{x}}_{k+1}^{i}$, covariance $\mathbf{P}_{k+1}^{i}$ and innovation covariance $\mathbf{S}_{k+1}^{i}$ are the outputs of the Kalman filter bank block which the relative equations presented in "Mode Matched Measurement Update" and "Mode Matched Prediction Update" in Algorithm 2.

The "mode probability calculation" block, processes $N$ previous mode probabilities, innovation covariances $\mathbf{S}_{k+1}^{i}$, and estimated GPS positions $\hat{\mathbf{y}}_{k+1 \mid k}^{i}$ to update the mode probability. The results of the filter bank are then passed to the "Mixing" and "Output estimation calculation" block. The latter block processes the mode probability and estimated states together with the error covariances, and other filter parameters to obtain the final filtering output consisting of a state vector estimate $\hat{\mathbf{x}}_{k+1}$ and its corresponding covariance $\mathbf{P}_{k+1}$. 
Algorithm 2 IMM Filter [86, 9]

- Input: previous sufficient statistics

- Mixing: Compute the mixing probability $\mu_{k}^{j i}$, mixed estimates $\hat{\mathbf{x}}_{k \mid k}^{0 i}$ and covariances $\mathbf{P}_{k \mid k}^{0 i}$ :

$$
\begin{aligned}
\mu_{k \mid k}^{j i} & =\frac{\pi_{j i} \mu_{k}^{j}}{\sum_{l=1}^{N} \pi_{l i} \mu_{k}^{l}}, \\
\hat{\mathbf{x}}_{k \mid k}^{0 i} & =\sum_{j=1}^{N} \mu_{k \mid k}^{j i} \hat{\mathbf{x}}_{k \mid k}^{j}, \\
\mathbf{P}_{k \mid k}^{0 i} & =\sum_{j=1}^{N} \mu_{k \mid k}^{j i}\left[\mathbf{P}_{k \mid k}^{j}+\left(\hat{\mathbf{x}}_{k \mid k}^{j}-\hat{\mathbf{x}}_{k \mid k}^{0 i}\right)\left(\hat{\mathbf{x}}_{k \mid k}^{j}-\hat{\mathbf{x}}_{k \mid k}^{0 i}\right)^{T}\right] .
\end{aligned}
$$

- Mode Matched Prediction Update: Compute the $\hat{\mathbf{x}}_{k+1 \mid k}^{i}$ and $\mathbf{P}_{k+1 \mid k}^{i}$

$$
\begin{aligned}
\hat{\mathbf{x}}_{k+1 \mid k}^{i} & =\mathbf{F}(i) \hat{\mathbf{x}}_{k \mid k}^{0 i}, \\
\mathbf{P}_{k+1 \mid k}^{i} & =\mathbf{F}(i) \mathbf{P}_{k \mid k}^{0 i} \mathbf{F}^{T}(i)+\mathbf{G}(i) \mathbf{Q G}{ }^{T}(i) .
\end{aligned}
$$

- Mode Matched Measurement Update: Compute the update estimate $\hat{\mathbf{x}}_{k+1 \mid k+1}$, covariance $\mathbf{P}_{k+1 \mid k+1}$ and update the mode probability $\mu_{k+1}^{i}$

$$
\begin{aligned}
\hat{\mathbf{y}}_{k+1 \mid k}^{i} & =\mathbf{H}(i) \hat{\mathbf{x}}_{k+1 \mid k}^{i}, \\
\mathbf{S}_{k+1}^{i} & =\mathbf{H}(i) \mathbf{P}_{k+1 \mid k}^{i} \mathbf{H}(i)^{T}+\mathbf{R}, \\
\mathbf{K}_{k+1}^{i} & =\mathbf{P}_{k+1 \mid k}^{i} \mathbf{H}^{T}(i)\left(\mathbf{S}_{k+1}^{i}\right)^{-1}, \\
\hat{\mathbf{x}}_{k+1 \mid k+1}^{i} & =\hat{\mathbf{x}}_{k+1 \mid k}^{i}+\mathbf{K}_{k+1}^{i}\left(\mathbf{y}_{k}-\hat{\mathbf{y}}_{k+1 \mid k}^{i}\right), \\
\mathbf{P}_{k+1 \mid k+1}^{i} & =\mathbf{P}_{k+1 \mid k+1}^{i}-\mathbf{K}_{k+1}^{i} \mathbf{S}_{k+1}^{i} \mathbf{K}_{k+1}^{i T}, \\
\mu_{k+1}^{i} & =\frac{\mathcal{N}\left(\mathbf{y}_{k+1} ; \hat{\mathbf{y}}_{k+1 \mid k}^{i}, \mathbf{S}_{k+1}^{i}\right) \sum_{j=1}^{N} \pi_{j i} \mu_{k}^{j}}{\sum_{l=1}^{N} \mathcal{N}\left(\mathbf{y}_{k+1} ; \hat{\mathbf{y}}_{k+1 \mid k}^{l}, \mathbf{S}_{k+1}^{l}\right) \sum_{j=1}^{N} \pi_{j l} \mu_{k}^{j}} .
\end{aligned}
$$

- Output Estimate Calculation: Compute the overall $\hat{\mathbf{x}}_{k+1 \mid k+1}$ and $\mathbf{P}_{k+1 \mid k+1}$

$$
\begin{aligned}
\hat{\mathbf{x}}_{k+1 \mid k+1} & =\sum_{i=1}^{N} \mu_{k+1}^{i} \hat{\mathbf{x}}_{k+1 \mid k+1}^{i}, \\
\mathbf{P}_{k+1 \mid k+1} & =\sum_{i=1}^{N} \mu_{k+1}^{i}\left[\mathbf{P}_{k+1 \mid k+1}^{i}+\left(\hat{\mathbf{x}}_{k+1 \mid k+1}^{i}-\hat{\mathbf{x}}_{k+1 \mid k+1}\right)\left(\hat{\mathbf{x}}_{k+1 \mid k+1}^{i}-\hat{\mathbf{x}}_{k+1 \mid k+1}\right)^{T}\right] .
\end{aligned}
$$

- Output: current sufficient statistics. 
The output calculation step in Algorithm 2 gives the output of the whole process. However, it does not affect the sufficient statistics for the next loop as illustrated in "output estimation calculation" block in Fig. 5.6.

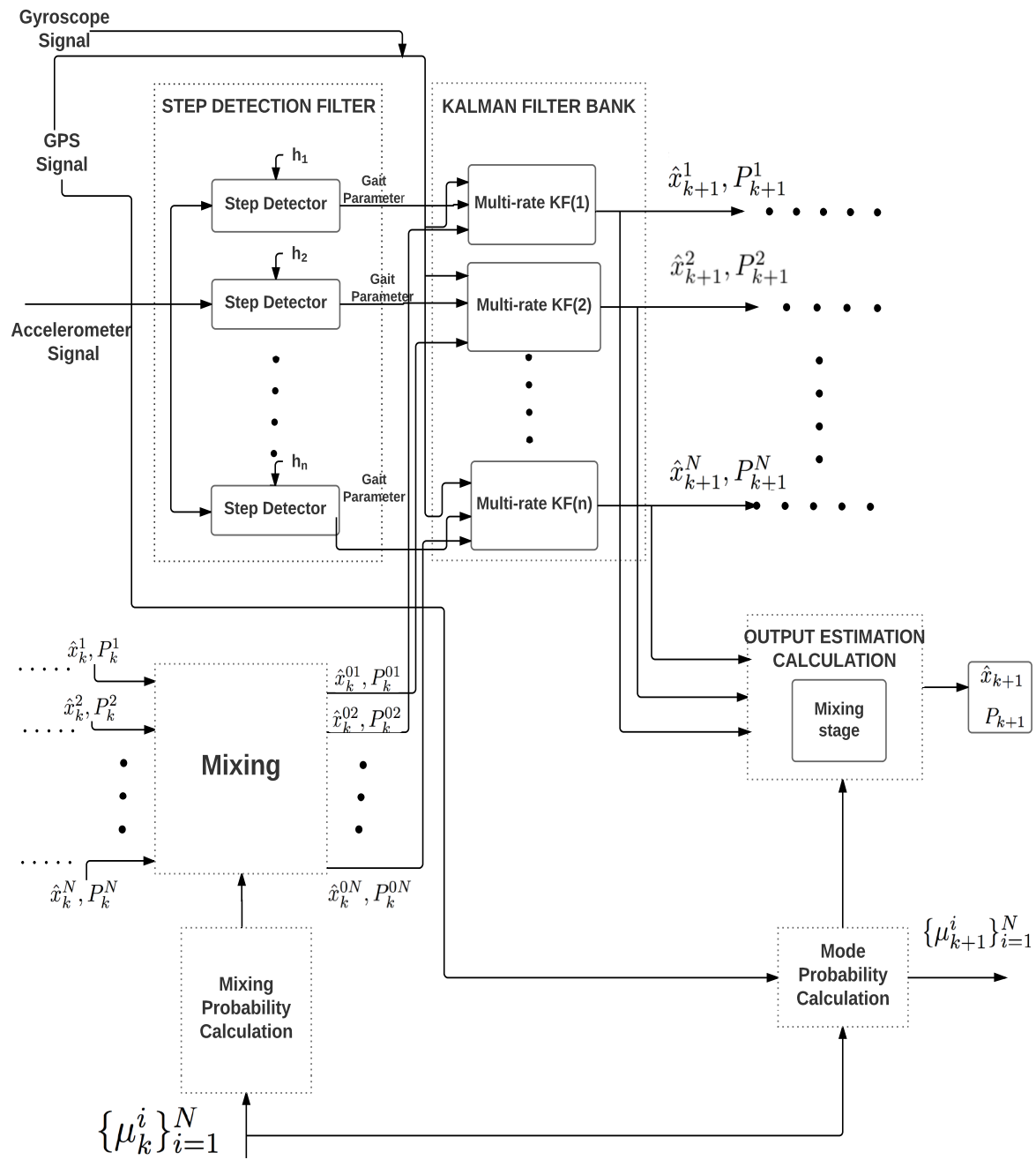

Figure 5.6: Block diagram of one step of the online Kalman filter bank using IMM algorithm for $N$ models. 


\subsection{Dead reckoning in different environments}

All the proposed approaches in this chapter are verified by both simulated data and real field experiments.

\subsubsection{Simulated Environment}

Generated data is used to verified the models under perfect conditions, noise free. It is also allows for using the true value for the parameter as reference and compare with the estimate.

\section{Longitudinal model}

The results of the simulated data for the longitudinal model are represented in the Fig. 5.7 and Fig. 5.8. Fig. 5.7 shows the mode probabilities and estimated step length for three different thresholds. The true step length in this scenario is 0.75 $\mathrm{m}$. The process noise covariance matrix for this model is $\mathbf{Q}=\operatorname{diag}([0.1,0.001])$ and the measurement noise for GPS and step length are $0.1 \mathrm{~m}$ and $0.01 \mathrm{~m}$, respectively. Setting a reasonable threshold results in accurate step length estimate. Meanwhile, applying a very low/high threshold makes the estimated quantities to diverge from true value. Fig. 5.7a indicates "reasonable threshold" with solid red line which has the highest probability and as shows in Fig. 5.7b the step length estimation is highly accurate.

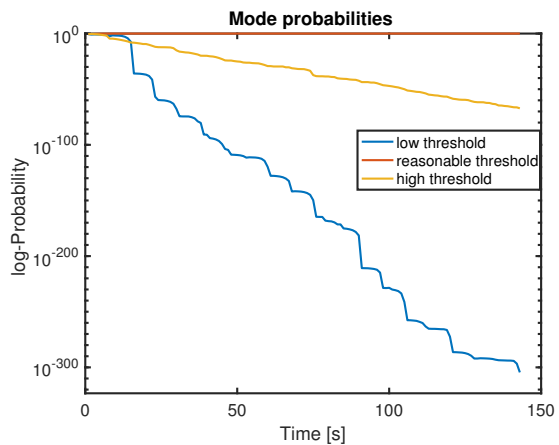

(a) Probability mode for three different thresholds

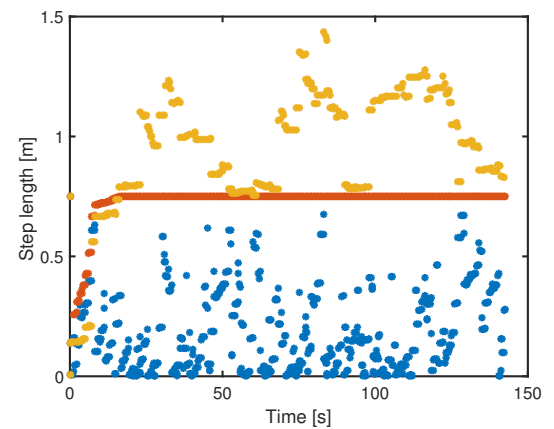

(b) Estimated step length for three different thresholds.

Figure 5.7: The results are the "Kalman Filter bank" block outputs represented in Fig. 5.6. The data is generated for the longitudinal model and the online Kalman filter bank approach is used.

By mixing the threshold results, the step lengths and the travelled distances are estimated accurately as illustrated in Fig. 5.8. Fig 5.8a represents the step length estimation results which is the output result of the "output estimation 


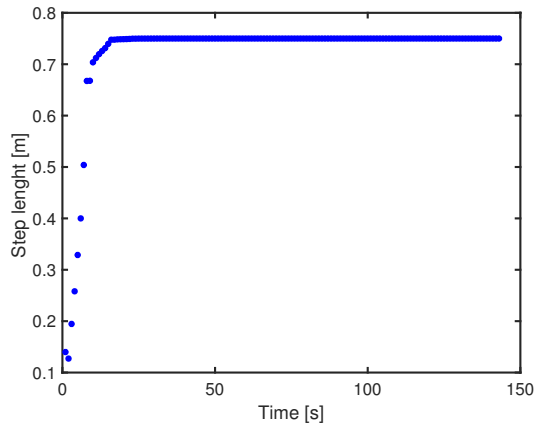

(a) Estimated step length by mixing hypothesis for the thresholds. The results is from the "output estimation calculation" block in Fig. 5.6.

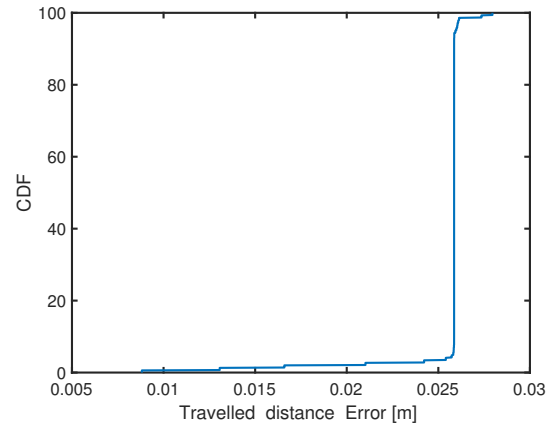

(b) The CDF of root mean square error for estimated travelled distance.

Figure 5.8: The data is generated for the longitudinal model and the online Kalman filter bank approach is used.

calculation" block in Fig. 5.6. Moreover, Fig. 5.8b represents the root mean square error of estimated travelled distance vs true travelled distance.

\section{Horizontal model}

Data was also generated to apply to the horizontal model. The true step length in this scenario is $0.75 \mathrm{~m}$. The process noise covariance matrix for this model is $\mathbf{Q}=\operatorname{diag}([10,0.1,0.0000001])$ and the measurement noise for GPS, gyroscope and step length are $0.1 \mathrm{~m}, 10$ and $0.001 \mathrm{~m}$, respectively. The thresholds are set with the same value as longitudinal model. In Fig. 5.9a the "reasonable threshold" indicated with solid red line represents the highest probability in Fig. 5.9b. The estimated step lengths for this threshold are the closest to the true step length.

By mixing the threshold results, the step lengths and position are estimated accurately as illustrated in Fig. 5.10. Fig. 5.10a represents the step length estimation results which is the output from the "output estimation calculation" block in Fig. 5.6. Moreover, Fig. 5.10b represents the estimated position over the true position where the estimated trajectory follows the true path. 


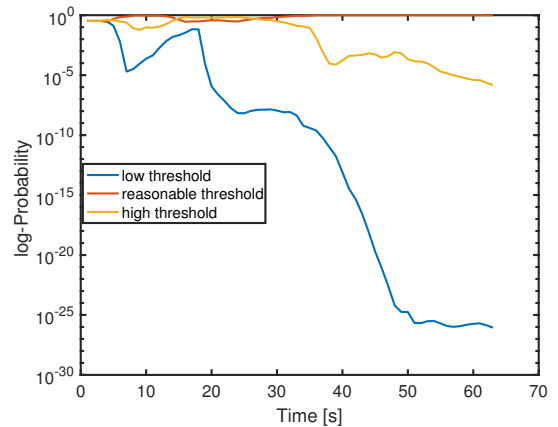

(a) Probability mode for three different thresholds.

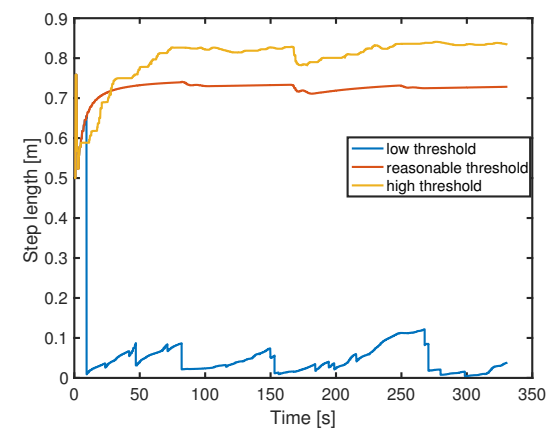

(b) Estimated step length for three different thresholds.

Figure 5.9: The results of the "Kalman Filter bank" block in Fig. 5.6. The data is generated for the horizontal model and using the online Kalman filter bank approach.

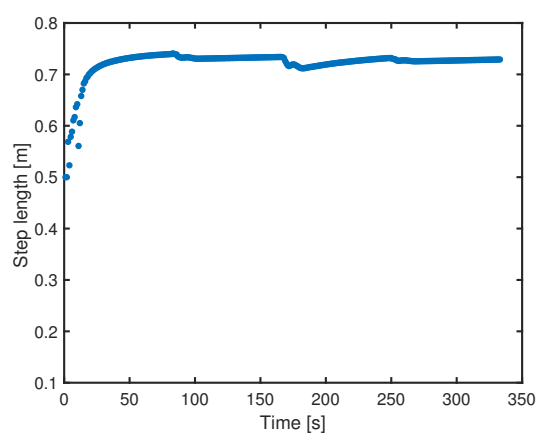

(a) Estimated step length by mixing hypothesis for the thresholds.

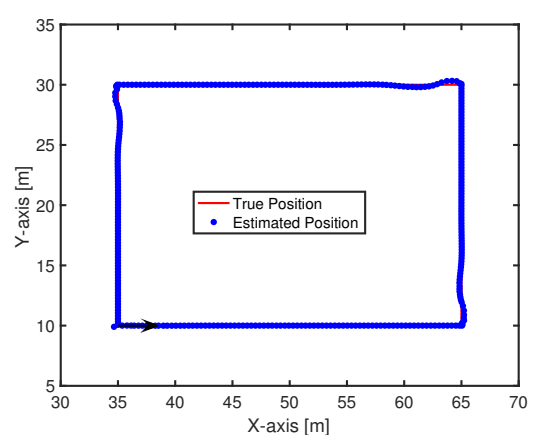

(b) Estimated position vs true position.

Figure 5.10: The results of the "output estimation calculation" block in Fig. 5.6. The data is generated for the horizontal model and using the online Kalman filter bank approach. 


\subsubsection{Real Environment}

The models proposed in Section 5.1.1 and [48] are evaluated using experimental data. Measurements were collected in a parking lot at Linköping university, using the sensor fusion Android app [57, 38] to log GPS, accelerometer and gyroscope measurements. Several experiments were conducted with people of different heights and weights. Each person repeated the experiment in different walking modes; walking, running and standing still. All the data were collected over the same trajectory that contains four sharp corners. Moreover, the cellphone were carried flat in the hand for all scenarios.

\section{Offline Kalman Filter bank results}

Table 5.2 presents the results from applying the longitudinal model described in Section. 5.1.1 to the collected data. The elements of the process noise covariance matrix for this model were selected as $\sigma_{a}^{2}=10\left(\mathrm{~m} / \mathrm{s}^{2}\right)^{2}$ and $\sigma_{L}^{2}=0.002 \mathrm{~m}^{2}$, and the measurement noise variances for GPS and step length are $\sigma_{\mathrm{v}, \mathrm{GPS}}^{2}=9(\mathrm{~m} / \mathrm{s})^{2}$ and $\sigma_{\text {step }}^{2}=0.04 \mathrm{~m}^{2}$, respectively. The results of adaptively determining the step detection threshold are compared to the results when fixing the threshold to suit user 1. In Table 5.2, the estimates are compared to the ground truth values for the gait parameters and the travelled distance. It shows that the estimates obtained with the fixed threshold for user 1 are comparable to those obtained with the adaptive scheme. However, the fixed threshold did work less well for the second user. In that case, choosing a too large step detection threshold results in too few steps being detected. Hence, the threshold that suits user 1 works less well with user 2. As can be seen, not only the number of steps are affected but also the step length adapts to satisfy the condition for the travelled distance.

Fig. 5.11 presents results using the horizontal model described in [48]. The elements of the process noise covariance matrix for this model were selected as $\sigma_{a}^{2}=10\left(\mathrm{~m} / \mathrm{s}^{2}\right)^{2}, \sigma_{\alpha}^{2}=0.5 \mathrm{rad}^{2}$ and $\sigma_{L}^{2}=0.002 \mathrm{~m}^{2}$, and the measurement noise variance for GPS was selected as $\sigma_{\mathrm{p}, \mathrm{GPS}}^{2}=9 \mathrm{~m}^{2}$, for gyroscope as $\sigma_{\text {gyro }}^{2}=0.64 \mathrm{rad}^{2}$ and for step length as $\sigma_{\text {step }}^{2}=0.04 \mathrm{~m}^{2}$. In this figure, the PDR estimation is shown with adaptive and fixed gait step detection threshold (again chosen to suit user 1 well) when two users walked slowly. The threshold for step detection has been estimated for Fig. 5.11a and Fig. 5.11c. Conversely, in Fig. 5.11e the fixed threshold is used and applied to the same measurement set as used in Fig. 5.11c. The GPS signal (green dots) is only assumed available for a few seconds in the beginning and middle of experiments. As can be seen in the figure, using the adaptive step detection threshold provides reasonably good position estimates even during periods without GPS coverage. At the same time, Fig. 5.11e shows how large influence poorly chosen gait parameters can have on the end result, when compared to adaptively choosing the parameters. It should be noted that in these limited experiments, simply choosing a very low step detection threshold would improve the result. However, we anticipate this not to be the case in more realistic settings with uneven gait and regular stops which motivates the choice of parameters here. 
Table 5.2: Estimated gait parameters and travelled distance versus true one [48, C2016 IEEE].

\begin{tabular}{|c|c|c|c|c|}
\hline & $\begin{array}{c}\text { Traveled } \\
\text { distance } \\
{[m]}\end{array}$ & $\begin{array}{c}\text { Number } \\
\text { Of } \\
\text { Steps }\end{array}$ & $\begin{array}{c}\text { Position } \\
\text { error at the } \\
\text { end point } \\
{[\mathrm{m}]}\end{array}$ & $\begin{array}{c}\text { Threshold } \\
{\left[\mathrm{m} / \mathrm{s}^{2}\right]}\end{array}$ \\
\hline $\begin{array}{c}\text { Ground Truth } \\
\text { User 1 }\end{array}$ & 248 & 333 & $\mathbf{0}$ & 2.06 \\
\hline $\begin{array}{c}\text { User 1 with } \\
\text { adaptive } \\
\text { gait parameters }\end{array}$ & 246.1 & 329 & 6.9 & 2 \\
\hline $\begin{array}{c}\text { User 1 with } \\
\text { fixed } \\
\text { gait parameters }\end{array}$ & 245.2 & 321 & 8.6 & 0.81 \\
\hline $\begin{array}{c}\text { Ground Truth } \\
\text { User 2 }\end{array}$ & 242 & 346 & $\mathbf{0}$ & 0.8 \\
\hline $\begin{array}{c}\text { User 2 with } \\
\text { adaptive } \\
\text { gait parameters }\end{array}$ & 240.1 & 352 & 9.8 & 2 \\
\hline $\begin{array}{c}\text { User 2 with } \\
\text { fixed } \\
\text { gait parameters }\end{array}$ & 227.7 & 141 & 32.8 & $\mathbf{0 . 8}$ \\
\hline
\end{tabular}

The step lengths estimated with adaptive threshold, Fig. 5.11d and Fig. 5.11b, are fairly constant. In this figure green lines indicate availability of the GPS signal, as in the previous case. Fig. 5.11f shows the negative effect on the step length when the steps are incorrectly detected due to a poor step detection threshold. 


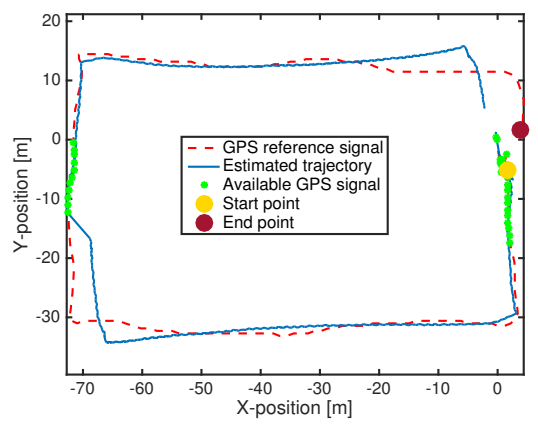

(a) User 1 with adaptive parameters.

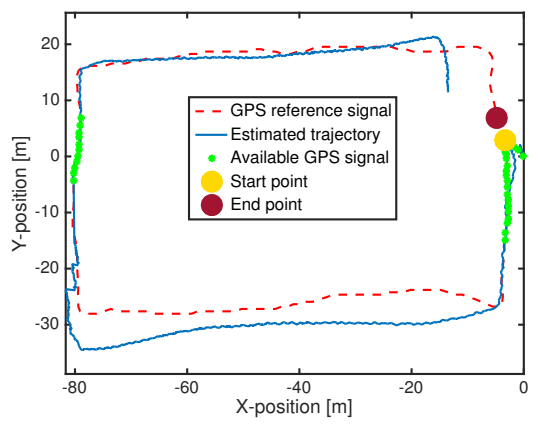

(c) User 2 with adaptive parameters.

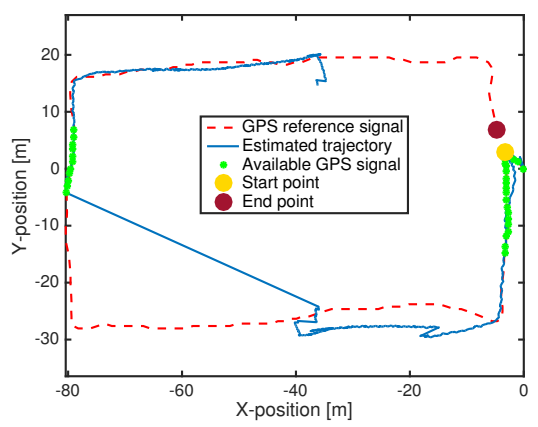

(e) User 2 with fixed parameters.

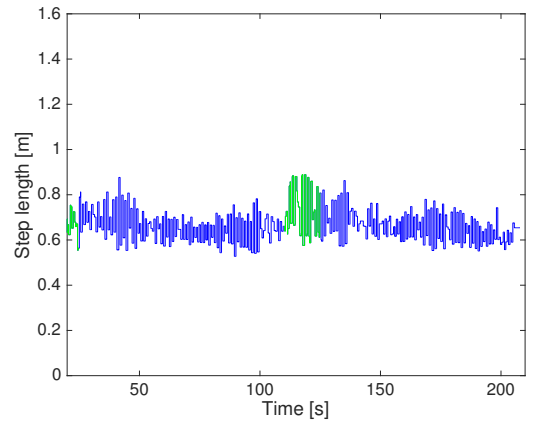

(b) The estimated step lengths, the green lines indicate time slots of available GPS signals.

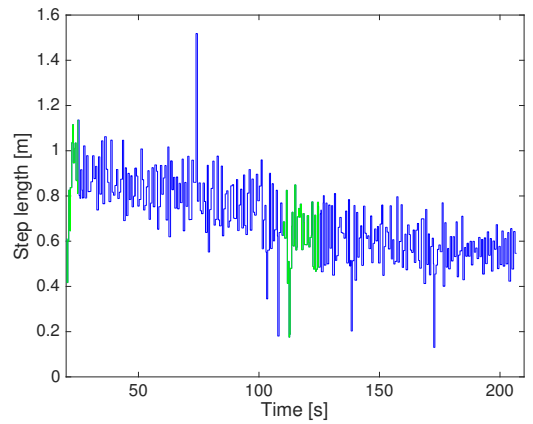

(d) The estimated step lengths, the green lines indicate time slots of available GPS signals.

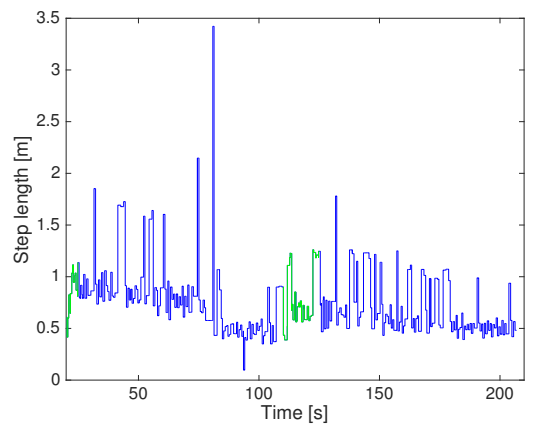

(f) The estimated step lengths, the green lines indicate time slots of available GPS signals.

Figure 5.11: Illustration of the estimated trajectory with PDR Vs. GPS reference trajectory. Green dots indicate where GPS signals were assumed available. The user starts walking South and in total makes four sharp turns until the end point is reached. People walked slowly and with constant speed during the data collection [48, (C2016 IEEE]. 


\section{Online Kalman Filter bank results}

The online Kalman filter bank results are presented in this section. The results of the longitudinal model are gathered for different walking modes; in the simple scenario, the user walks with constant pace during the whole measurement, while complex scenarios mimic a more realistic application in which users change their motion mode between walking, standing still, and running. In the following figures, different modes are distinguished by their initial letter; "W" stands for walking, "SS" for standing still and " $\mathrm{R}$ " for running. The process noise covariance matrix for this model is $Q=\operatorname{diag}([10,0.00000001])$ and the measurement noises, standard deviations, for the GPS and step length are $0.2 \mathrm{~m}$ and $0.01 \mathrm{~m}$, respectively. The TPM matrix is a $3 \times 3$ identity matrix since three hypotheses are used in this scenario. The results obtained for the simple walking mode are summarized in Fig. 5.12. Fig. 5.12a and Fig. 5.12b represent the mode probability with and without GPS signal for three different thresholds. According to these figures, the mode probability for the second threshold has the highest value that is very well in-line with our expectations compared to the other two thresholds that stand for extreme low and high values. The results of estimated step lengths for these two situations (with GPS and GPS denied), are illustrated in Fig. $5.12 \mathrm{c}$ and Fig. 5.12d, respectively. The step length estimate remains constant for all measurements in both situations, as expected since the user is always walking with constant speed throughout the whole experiment. The step lengths are estimated to be around $0.8 \mathrm{~m}$ which is very reasonable for a normal person.

The travelled distance estimation error for both environments is computed and illustrated using a cumulative distribution function (CDF) in Fig. 5.12e and Fig. 5.12f. According to these figures, the travelled distance is estimated with rather low error, even when the GPS is not always available. The ground truth is calculated from position measurements provided by the GPS.

The complex walking pattern in the longitudinal model is also evaluated and the results are reported in Fig 5.13. Similar conclusions as in the simple walking mode are valid in these scenarios as well. As these figures show, the PDR with gait parameter learning gives good estimation for step length and travelled distance even during periods without GPS coverage. 


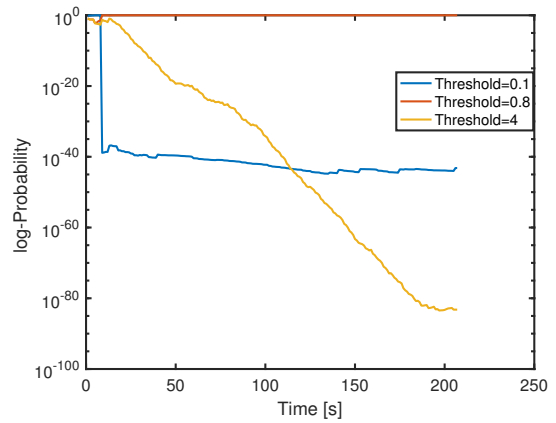

(a) Mode probability for three different thresholds. GPS is available during entire experiment.

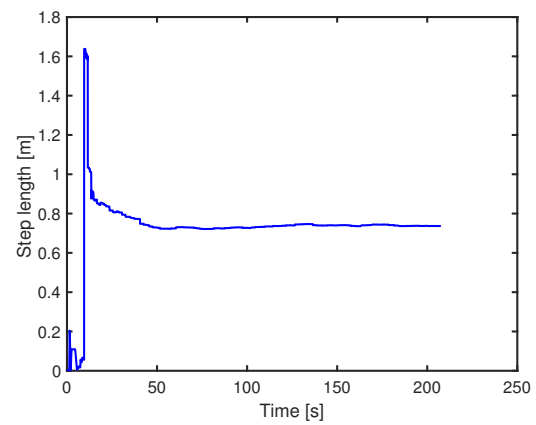

(c) The estimated step length. GPS is available during entire experiment.

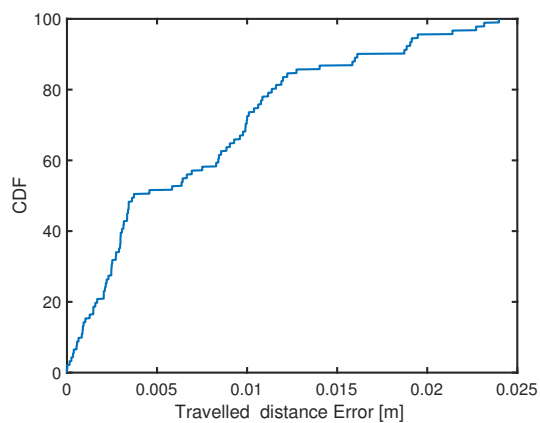

(e) The CDF of root mean square error for estimated travelled distance. GPS is available during entire experiment.

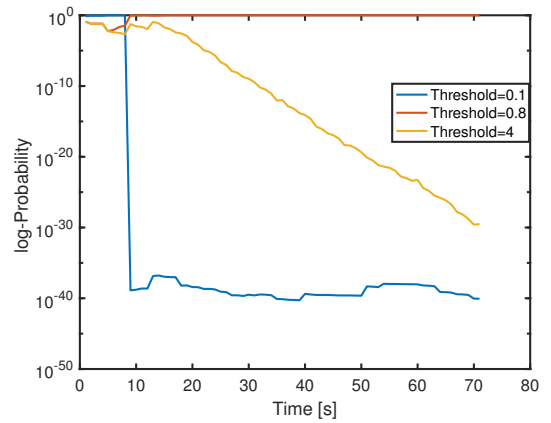

(b) Mode probability for three different thresholds in GPS denied environment.

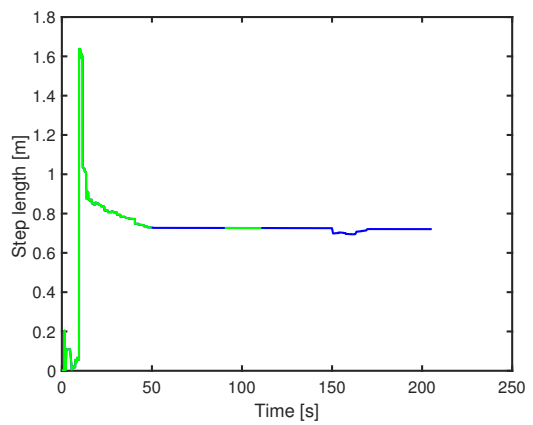

(d) The estimated step length in GPS denied environment. Green solid line indicate where GPS signals were assumed available.

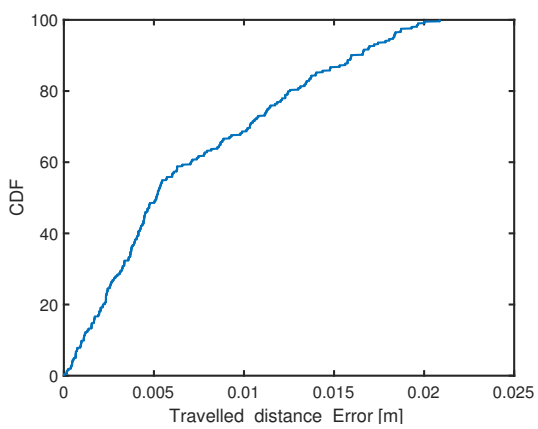

(f) The CDF of root mean square error for estimated travelled distance in GPS denied environment.

Figure 5.12: Results for longitudinal model with real data for simple walking pattern. 


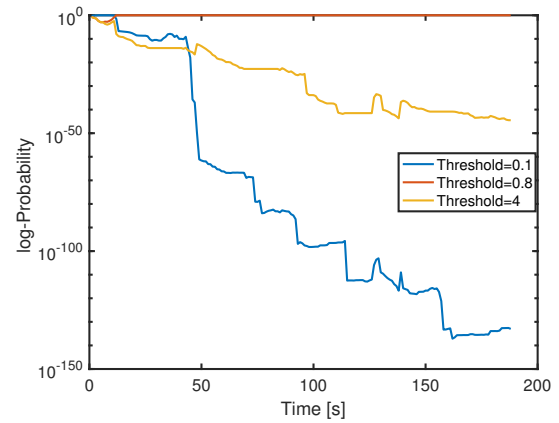

(a) Mode probability for three different thresholds. GPS is available during entire experiment.

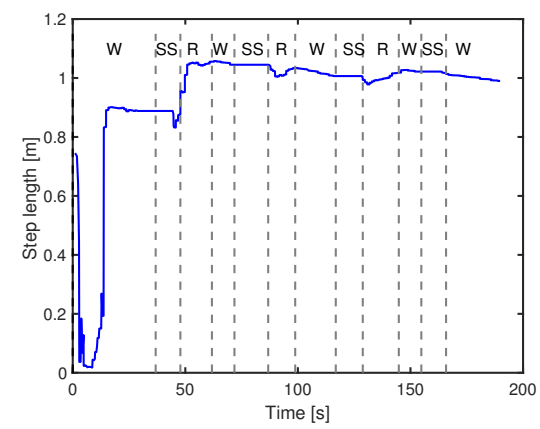

(c) The estimated step length. GPS is available during entire experiment.

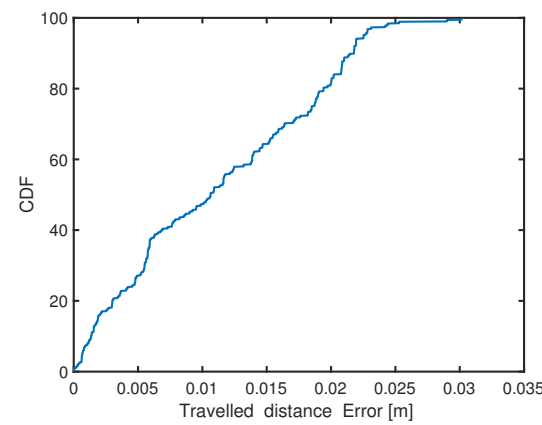

(e) The CDF of root mean square error for estimated travelled distance with GPS signal always available during entire experiment.

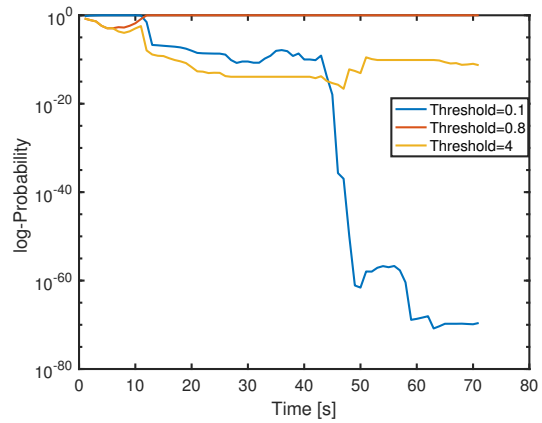

(b) Mode probability for three different thresholds in GPS denied environment.

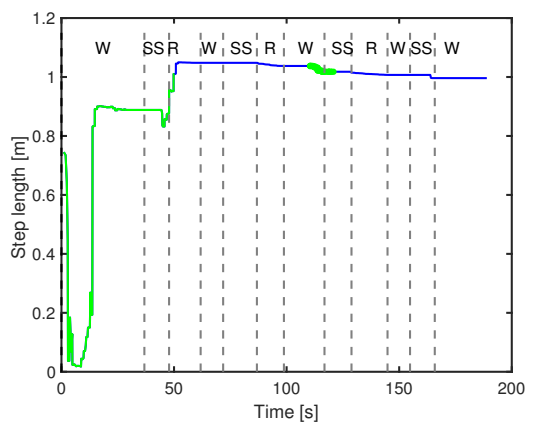

(d) The estimated step length in GPS denied environment. Green solid line indicate where GPS signals were assumed available.

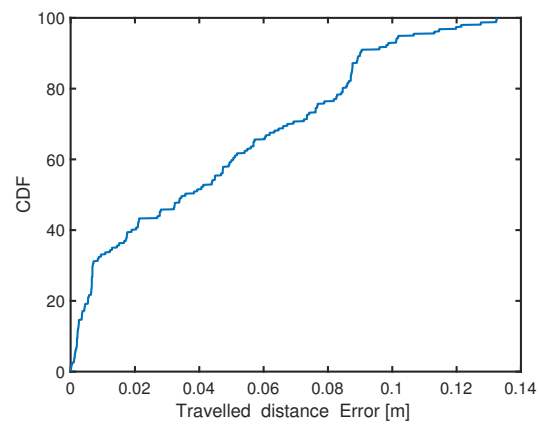

(f) The CDF of root mean square error for estimated travelled distance in GPS denied environment.

Figure 5.13: Results for longitudinal model with real data for complex walking pattern. 
The results for the horizontal model are presented in both simple and complex walking patterns. The process noise covariance matrix for this model is $Q=\operatorname{diag}([100,0.8,0.0000001,0.00001]$ and the measurement noises standard deviations for GPS is $10 \mathrm{~m}$, for gyroscope is $0.8 \mathrm{rad}$ and for step length is 0.01 $\mathrm{m}$. The ТРМ matrix is $3 \times 3$ identity matrix.

The result of the mode probability, step length estimation and estimated position form the horizontal model with simple walking mode for both GPS and GPS denied environments are illustrated in Fig. 5.14. Fig. 5.14a and Fig. 5.14b represent the mode probability with and without GPS signals, respectively. As these figures show, the highest mode probability is for the threshold indicated with red solid line which has a reasonable value since the user is walking with constant speed during this experiment. Fig. 5.14c and Fig. 5.14d illustrate the step length estimation for both GPS and GPS denied situations. The estimated step length is constant and around $0.85 \mathrm{~m}$ which is close to the estimated step length by the longitudinal model. The estimated step length in GPS denied environment is almost the same as the one that has been estimated when GPS is always available. Fig. 5.14e shows the estimated position when GPS is available for the whole measurements where the walking path estimation has a high accuracy. In Fig. 5.14f, the GPS is only available for a short periods, indicated with green dots. The estimation follows the trajectory but with a small drift.

Fig. 5.15 represents the horizontal model with the complex walking mode for the same environments; with GPS signal and GPS denied. Fig. 5.15a and Fig. 5.15b represent the mode probability with and without GPS signal. As these figures show, the threshold with value one, has the highest probability mode. Fig. 5.15c and Fig. 5.15d show the estimated step length for the horizontal model with complex walking mode. As can be seen in these figures, the estimated step length does not have a constant trend because of the varying walking modes. The walking mode is indicated in the figures and the estimated step length increases and decreases in accordance with the change in the walking mode and thus the fluctuation in the estimated step length appears. Fig. 5.15e and Fig. 5.15f represent the position estimation for the GPS and GPS denied situations. Highly accurate position estimates when GPS is always available and a drift in the estimates when GPS availability is limited, as in the simple walking mode case, can be observed. 


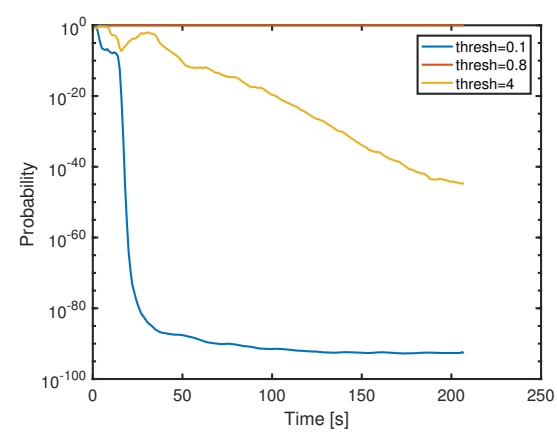

(a) Mode probability for three different thresholds. GPS is available during entire experiment.

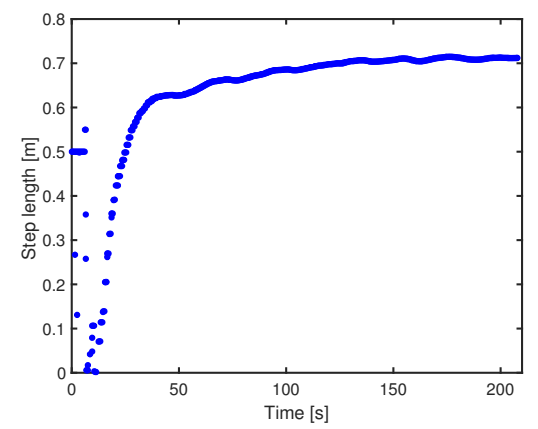

(c) The estimated step length. GPS is available during entire experiment.

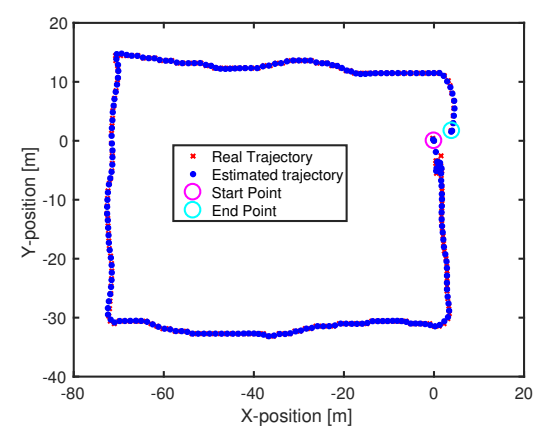

(e) The estimated position vs true position. GPS is available during entire experiment.

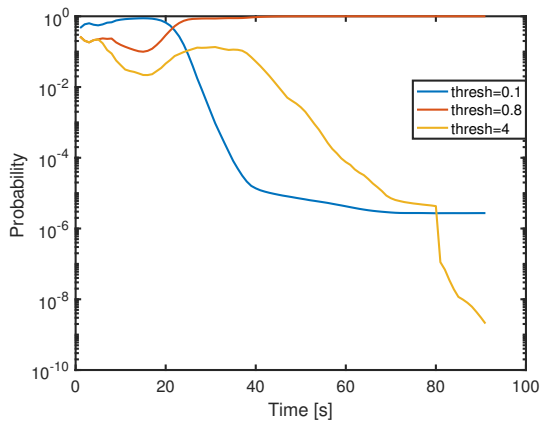

(b) Mode probability for three different thresholds in GPS denied environment.

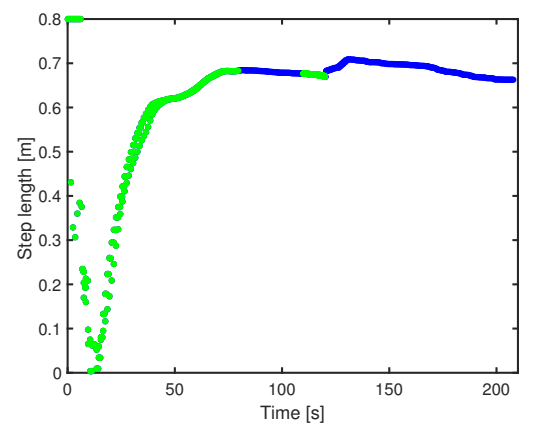

(d) The estimated step length in GPS denied environment. Green dots indicate where GPS signals were assumed available.

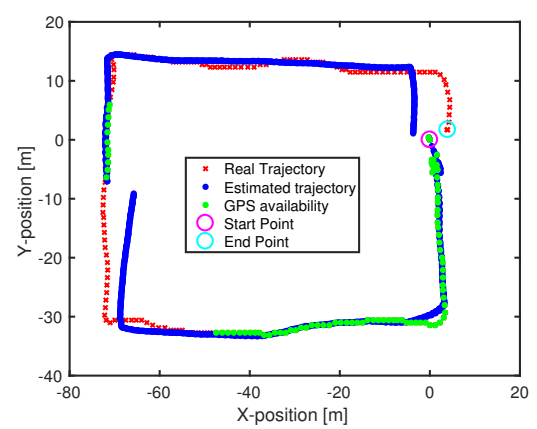

(f) The estimated position vs true position in GPS denied environment. Green dots indicate where GPS signals were assumed available.

Figure 5.14: Results for horizontal model with real data for simple walking pattern. 


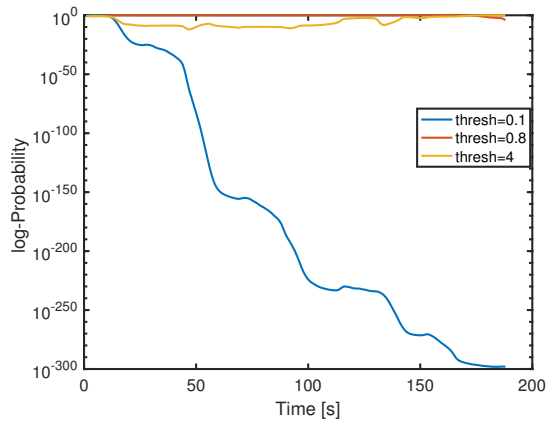

(a) Mode probability for three different thresholds. GPS is available during entire experiment.

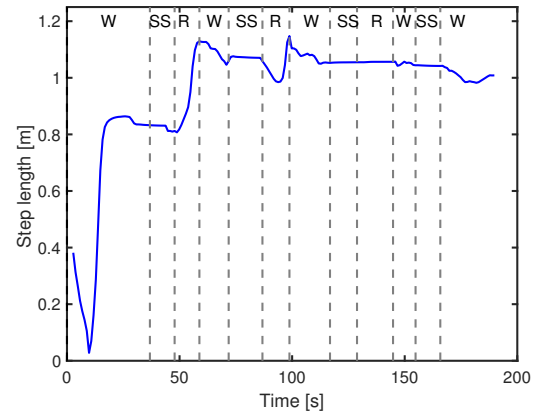

(c) The estimated step length. GPS is available during entire experiment.

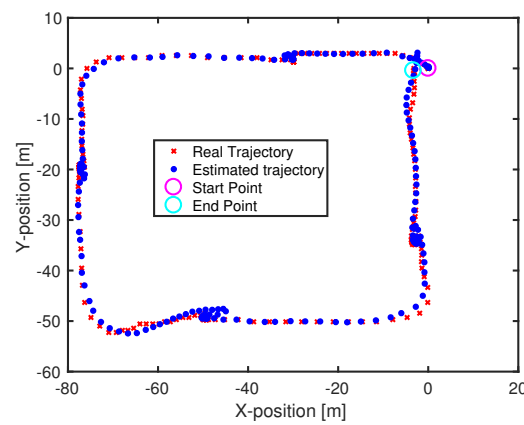

(e) The estimated position vs true position. GPS is available during entire $e^{-}$ periment.

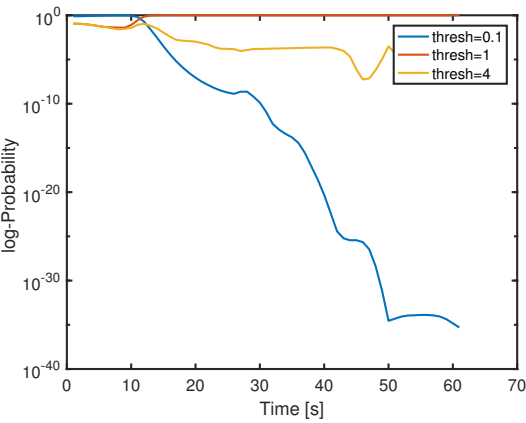

(b) Mode probability for three different thresholds in GPS denied environment.

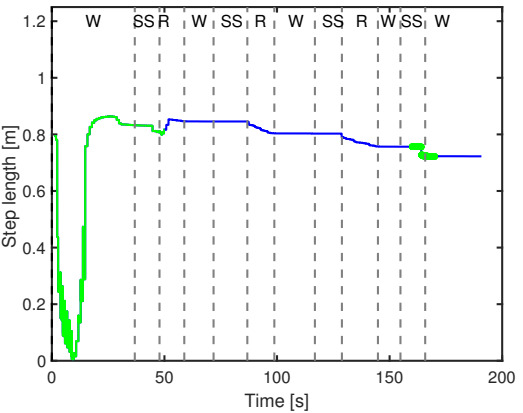

(d) The estimated step length in GPS denied environment. Green dots indicate where GPS signals were assumed available.

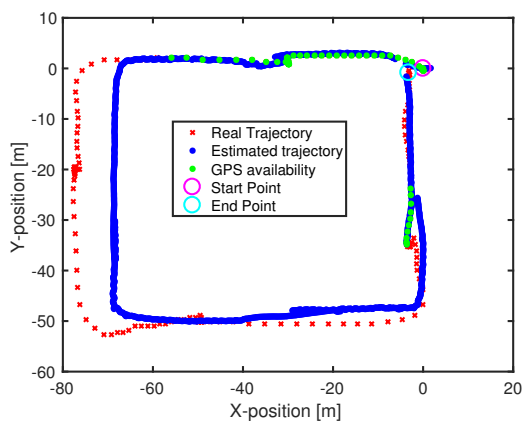

(f) The estimated position vs true position in GPS denied environment. Green dots indicate where GPS signals were assumed available.

Figure 5.15: Results for horizontal model with real data for complex walking pattern. 



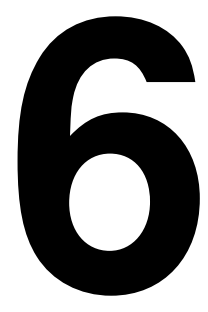

\section{Motion and Device Mode Classification}

In this chapter, we study human motion and device mode classification. Classification of motion mode (walking, running, standing still) and device mode (hand-held, in pocket, in backpack) is an enabler in personal navigation systems (PNS) for the purpose of saving energy and design parameter settings and also for its own sake. This application and its proposal solution were accepted to publish in (IPIN2017) [49, C2017 IEEE].

Our main contribution is to publish one of the most extensive datasets for this problem, including inertial data from eight users, each one performing three pre-defined trajectories carrying four smartphones and seventeen inertial measurement units on the body. All kind of metadata is available such as the ground truth of all modes and position. A second contribution is the first study on a joint classifier of motion and device mode, respectively, where preliminary but promising results are presented.

The rest of the chapter is organized as follows: Section 6.1 explains experimental setup in detail, followed by a description of available data in Section 6.2. In Section 6.3 data analysis and initial classification results are presented.

\subsection{Experiment Setup}

Our investigation is based on an extensive experimental study where different users repeated the same trajectories and sequences of modes. We logged data from low cost micro-electromechanical systems (MEMS) sensors including acceleration, angular rate, magnetic field, barometric pressure and also GPS as a position reference. Data were measured by using four smartphones and 17 inertial measurement units (IMUs) configured in a body suit. The IMUs generate data of somewhat higher accuracy than the smartphones, and the body suit software makes use of advanced biomechanical models to provide accurate description of 
true motion of all body parts, which in turn could be used to simulate data from any other part of the body. Ground truth of the device and motion mode is available from the experimental setup. Table 6.1 summarizes different device and motion modes. In order to simplify referring to each of these scenarios, Table 6.1 also assigns a specific class to each of them. We believe this to be one of the most extensive datasets publicly available for PNS.

Table 6.1: Motion-Device mode Classification [49, (C2017 IEEE].

\begin{tabular}{|c|c|c|c|}
\hline Device Mode $\quad \begin{array}{c}\text { Motion } \\
\text { Mode }\end{array}$ & $\begin{array}{c}\text { Standing Still } \\
(\mathrm{SS})\end{array}$ & $\begin{array}{l}\text { Walking } \\
\text { (W) }\end{array}$ & $\begin{array}{l}\text { Running } \\
\text { (R) }\end{array}$ \\
\hline Fixed hand (1) & \multirow{4}{*}{ Class SS } & Class W1 & Class R1 \\
\hline Swinging hand (2) & & Class W2 & Class R2 \\
\hline Pocket (3) & & Class W3 & Class R3 \\
\hline Backpack (4) & & Class W4 & Class R4 \\
\hline
\end{tabular}

In this section, we first introduce the hardware used in the experiments, then all measurement scenarios are described in detail. Finally, characteristics of all participants are presented to give a better comparison on signal behavior for different subjects with different attributes.

\subsubsection{Sensors}

The hardware can be grouped into two categories; high and low quality; MVN and Nexus 5, respectively. Subsequently, the signals from the mobile phones will be compared with the MVN system to have a better classification for mobile signals.

\section{Xsens MVN Motion Capture}

The Xsens MVN system has been used to capture the whole body motion. In the experiments the "MVN Awinda" system has been used. It contains 17 wireless Motion Trackers (MTw), an Awinda station, and MTw full body velcro straps.

The MTw is a miniature inertial measurement unit containing a 3-D linear accelerometer, a 3-D rate gyroscope, a 3-D magnetometer, and a barometer.

The 17 trackers are placed at strategic locations on the body (secured by the straps), to measure motion of each body segment. Fig. 6.1 shows the location of the straps and attached MTw. The MVN system is controlled by the MVN studio software. A snapshot of the MVN system is shown in Fig. 6.2.

\section{Nexus 5}

Four Nexus 5 smartphones are carried by the subjects in the experiments. The Nexus 5 is equipped with multiple sensors; The sensors that are considered in this work are the A-GPS, the 3-D linear accelerometer, the 3-D rate gyroscope, and the barometer. 


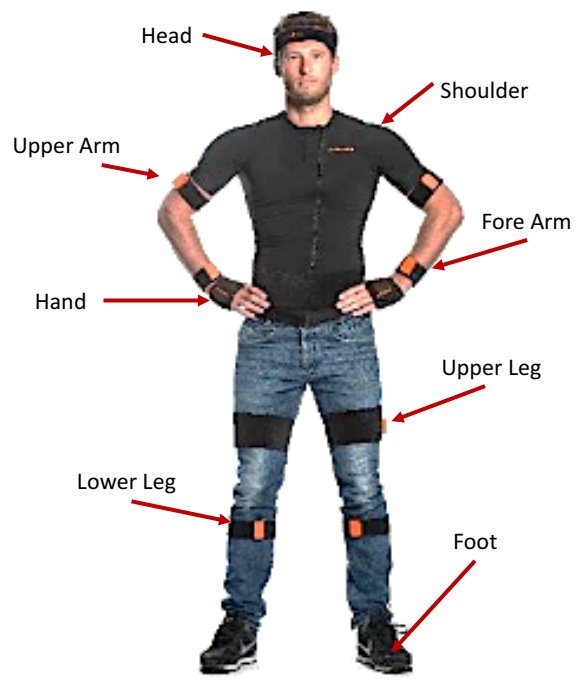

Figure 6.1: MVN Awinda Straps by courtesy of Xsens [49, C2017 IEEE].

The sensor fusion Android app [57, 39], installed on the Nexus 5 phones, is used to log the sensors measurements from the phones.

All phone locations used in the experiment are presented in Table 6.1. A summary of the details about both the MVN system and the phone is provided in Table 6.2; application, sampling frequency, sensor's positions, and IMU sensors.

Table 6.2: Measurement device specifications [49, (C2017 IEEE].

\begin{tabular}{|c|c|c|c|c|}
\hline Device & Application & $\begin{array}{c}\text { Sampling } \\
\text { Frequency } \\
{[\mathrm{Hz}]}\end{array}$ & Position & Sensors \\
\hline Xsens & $\begin{array}{c}\text { MVN } \\
\text { Awinda }\end{array}$ & 60 & $\begin{array}{c}\text { Head } \\
\text { Shoulders } \\
\text { Upper Arms } \\
\text { Fore Arms } \\
\text { Upper Legs } \\
\text { Lower Legs } \\
\text { Hands } \\
\text { Feet } \\
\text { Pelvis }\end{array}$ & $\begin{array}{c}\text { Accelerometer } \\
\text { Gyroscope } \\
\text { Magnetometer } \\
\text { Barometer }\end{array}$ \\
\hline Nexus 5 & $\begin{array}{l}\text { Sensor } \\
\text { Fusion } \\
\text { app }\end{array}$ & 100 & $\begin{array}{c}\text { Hand } \\
\text { Front Pocket } \\
\text { Back Pocket } \\
\text { Backpack }\end{array}$ & $\begin{array}{c}\text { GPS } \\
\text { Accelerometer } \\
\text { Gyroscope } \\
\text { Magnetometer } \\
\text { Barometer }\end{array}$ \\
\hline
\end{tabular}




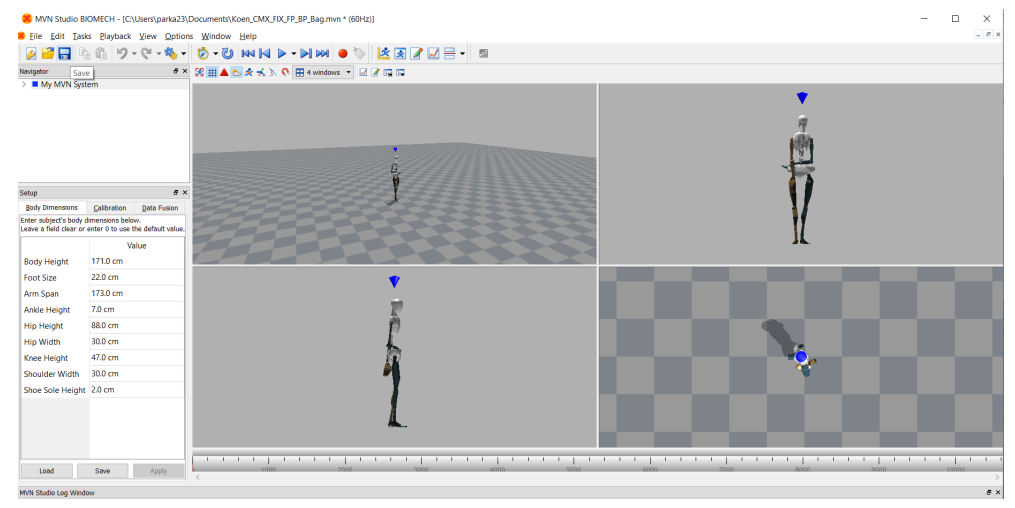

Figure 6.2: MVN Studio BIOMECH application [49, C2017 IEEE].

\subsubsection{Scenarios}

Measurements were collected in a building at Twente university. During the experiments the subjects walked three different paths, with a mixture of different motion modes, as represented in Table 6.1. We followed certain rules when gathering the data so that the obtained measurements should mimic reality. For instance, to avoid any abnormal behavior the subjects were asked to carry one smartphone in the hand at each time. One run-through of the scenarios including a preparation phase lasts around 45-60 min.

Fig. 6.3 illustrates three different paths on top of the map of the area where measurements were obtained. The paths on the maps are for illustrative purposes and are inexact. For each path in Fig. 6.3, the subject holds one mobile phone in the hand, two more phones in front and back pockets, and one in the backpack. In this section, we thoroughly present the three measurement scenarios studied in this work. One scenario contains outdoor-only measurements, while the other two scenarios combine both outdoor and indoor paths. All three scenarios share the property that the measurement begins and ends at the same point, in an outdoor environment.

\section{Outdoor-only, Standing Still, Walking and Running}

The simplest scenario corresponds to Fig. 6.3a where the whole measurement is performed outdoors where the GPS signal is available. In this scenario, measurements are performed while the subject covers several motion and device modes, corresponding to Case 1 in Table 6.3.

\section{Outdoor-indoor, Walking}

As in the previous scenario, the measurement starts and ends at the same point outside the building. However, a bit in to the track, the subject gets into the building and walks across a corridor, as illustrated in Fig. 6.3b. In this scenario, 


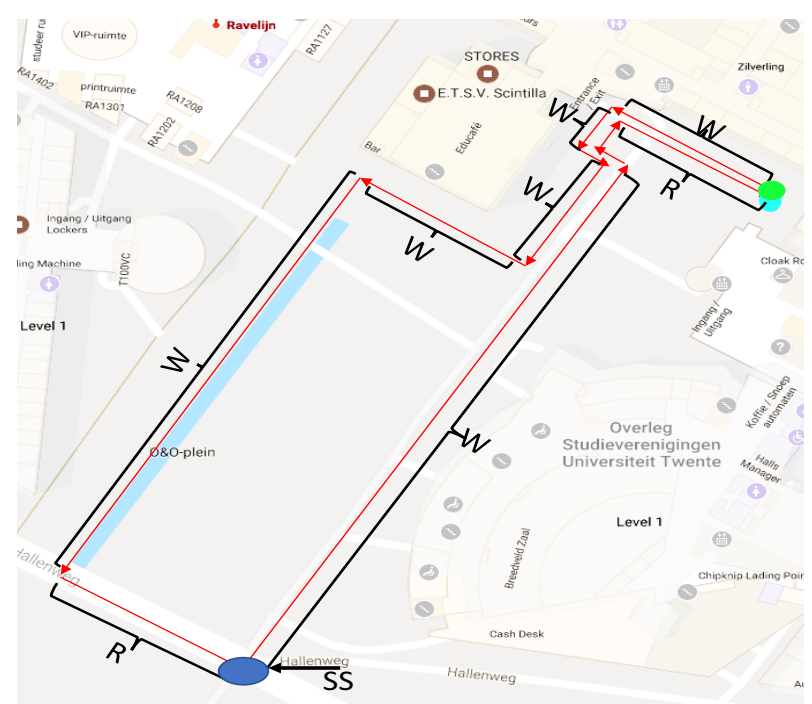

(a) Outdoor-only scenario with all classes (Case 1).

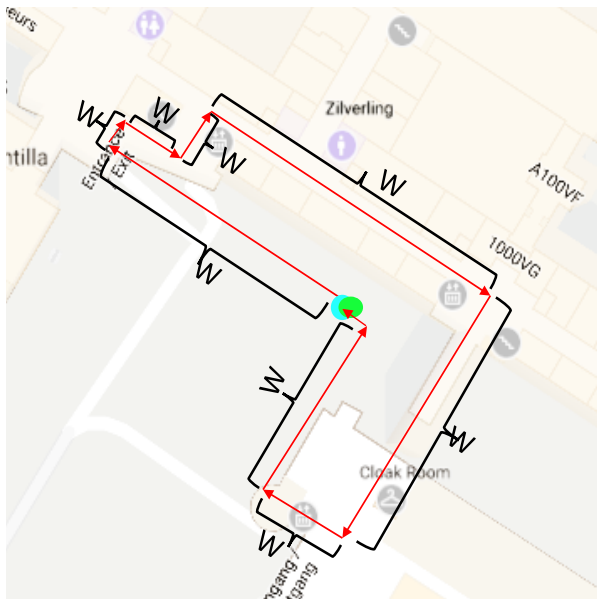

(b) Outdoor-Indoor scenario containing the W1 class (Case 2).

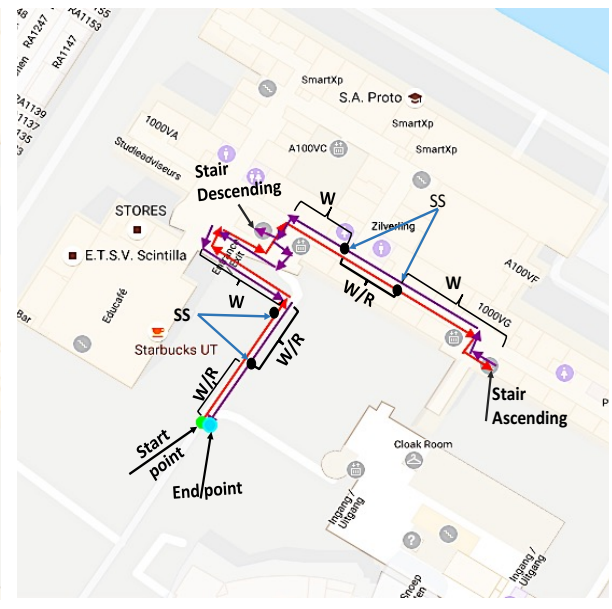

(c) Outdoor-Indoor scenario containing all classes. Forward and backward paths are indicated with red and purple colors, respectively (Case 3 and Case 4).

Figure 6.3: Different measurement scenarios. The start and end points are indicated by green and cyan dots, respectively. All the motion modes and classes are defined based on Table 6.1 [49, (C2017 IEEE].

measurements are performed while the subject walks the whole path and holds the smartphone flat and fixed in the hand, corresponding to Case 2 in Table 6.3. 


\section{Outdoor-indoor, Standing Still, Walking and Running}

In this scenario, we consider the most complex behavior in terms of motion modes and also the experiment path. Along the path depicted in Fig. 6.3c, the subject starts outside the building, then gets into the building and passes one corridor, takes the stairs up and passes a certain distance, followed by taking the stairs down and getting back to the starting point. The designed path in this scenario, is measured for two different cases:

- Case 3: The subject walks along the path for both sets of device modes presented in Table 6.3 (each set is performed separately).

- Case 4: The subject has several motion modes along the path for both sets of device modes presented in Table 6.3 (each set is performed separately).

\subsubsection{Participants}

The described experiments were performed by twelve volunteers, 7 males and 5 females, with ages ranging from 25 to 45 years old. Due to some technical and practical issues (GPS signal loss outdoors, physical difficulties making it impossible for subjects to perform all the experiments), only 6 men and 2 women performed all the explained scenarios.

\subsection{Available Data}

This section provides a detailed explanation of how the data was collected followed by a description of the ground truth. Finally, we clarify the data structure as well as provide instructions on accessing different parts of the available data summarized in Table 6.3.

\subsubsection{Collected Data}

Data is gathered from the scenarios in Sec. 6.1.2 using the hardware specified in Sec. 6.1.1. Before each experiment/data collection, the MVN Awinda is calibrated. The calibration can be done by MVN studio in a less disturbed magnetic field area while the subject is standing in a fixed pose for around 5 seconds. Table 6.3 summarizes all the different scenarios. It is worth noting that the laptop connected to the Awinda station must be near the subjects while recording measurements due to short signal range. Fig. 6.4 shows a subject while doing an experiment. She is wearing Xsens suit and carrying several phones.

The available data for both smartphone and Xsens suits together with units of each measurement are presented in Table 6.4. Both the raw sensory data as well as the virtual IMU measurements from the Biomechanical (BM) model are extracted from MVN studio and provided in the dataset. Although MTws are equipped with a barometer, the MVN studio cannot extract this feature, therefore, the MTw data for the barometer is not used. 
Table 6.3: Measurement scenarios. Device and motion modes are presented in Table 6.1. Paths corresponding to each case are depicted in Fig. 6.3 [49, (C) 2017 IEEE].

\begin{tabular}{|c|c|c|c|c|}
\hline Scenario & $\begin{array}{c}\text { Device } \\
\text { Mode }\end{array}$ & $\begin{array}{c}\text { Motion } \\
\text { Mode }\end{array}$ & Participants & $\begin{array}{c}\text { Duration } \\
\text { (average) } \\
{[\mathbf{s}]}\end{array}$ \\
\hline Case 1 & $1,2,3,4$ & W,R,SS & $\begin{array}{c}5 \text { Males } \\
\text { 2 Females }\end{array}$ & 190 \\
\hline Case 2 & $1,3,4$ & $\mathrm{~W}$ & $\begin{array}{c}6 \text { Males } \\
\text { 2 Females }\end{array}$ & 200 \\
\hline Case 3 & $\begin{array}{c}1,3,4 ; \\
2,3,4\end{array}$ & $\begin{array}{c}\text { Wpstairs } \\
\text { Downstairs }\end{array}$ & $\begin{array}{c}\text { 6 Males } \\
\text { 2 Female }\end{array}$ & 280 \\
\hline Case 4 & $\begin{array}{c}\text { W, } 3,4 ; \\
2,3,4\end{array}$ & $\begin{array}{c}\text { Upstairs } \\
\text { Downstairs }\end{array}$ & $\begin{array}{c}\text { 6 Males } \\
\text { 2 Female }\end{array}$ & 270 \\
\hline
\end{tabular}

The phone data is only available in raw version. The GPS signal from the phones is converted to East-North-Up (ENU) coordinates, with the first measured GPS position considered as the reference point.

\subsubsection{Ground Truth}

The ground truth plays an important role in the classification process. The classifier uses part of the data for which the corresponding class is known as training data in order to establish the discriminating criteria. The part of the ground truth which was not used for training purposes will afterwards be used for the assessment of the classification accuracy. The remaining data with unknown class could then be assigned to a class.

The classification accuracy refers to the correspondence between the class label assigned to each sample and the "true" class obtain by the ground truth. Fuzzy class boundaries and incorrectly assigned classes are two main degrading factors of classification accuracy. Additionally, if the ground truth does not represent all classes adequately the classification result and the corresponding accuracy may be unpredictable.

As Fig. 6.2 shows, MVN studio provides a movie of all the subject activities. We visually inspect these movies to determine the time of switch between the modes. However, the provided output of the MVN studio is a downsampled version of the signal. Thus, the visually obtained times need to be matched by the samples of the signal. We use the extracted output of the MVN studio that gives the exact time of each sample with milisecond(s) accuracy. All the samples are then labeled to appropriate classes using the derived time stamps used to form the ground truth.

The ground truth is separately formed for high and low quality devices using 


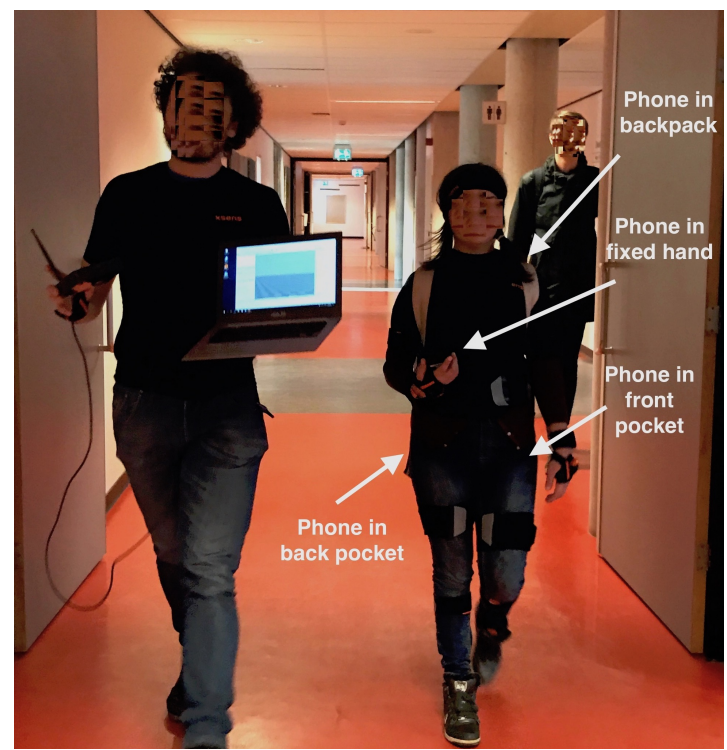

(a) The subject is walking constantly with almost constant speed and carrying a smartphone in flat and fixed hand.

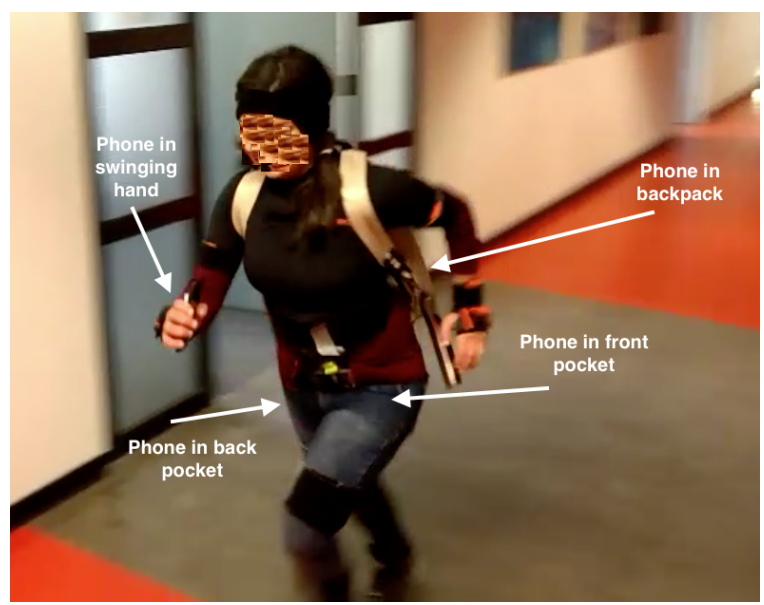

(b) The subject is running and carrying phone in swinging hand.

Figure 6.4: Photo from measurement campaign [49, O2017 IEEE].

the labels obtained. Depending on the device mode, signals obtained from appropriate MTws should be considered. For example, if the goal is to investigate the scenario in which the smartphone is being carried in hand, the hand-mounted sensor is the one that mimics that behaviors best. Another example is the case 
Table 6.4: Dataset details given from both smartphone and MTws. Signals from MTws are available in two versions; "Raw" data and filtered by some Biomechanical algorithms (BM). Smartphone data is only available in "Raw" version [49, C2017 IEEE].

\begin{tabular}{|c|c|c|c|}
\hline Variable Names & Unit & MTw data & Phone data \\
\hline 3D AccR (Raw) & {$\left[\mathrm{m} / \mathrm{s}^{2}\right]$} & ${ }^{*}$ & ${ }^{*}$ \\
\hline 3D AngVelR(Raw) & {$[\mathrm{rad} / \mathrm{s}]$} & ${ }^{*}$ & ${ }^{*}$ \\
\hline 3D OriR (Raw) & {$\left[{ }^{\circ}\right]$} & ${ }^{*}$ & ${ }^{*}$ \\
\hline 3D MagR (Raw) & {$[\mathrm{Gauss}]$} & ${ }^{*}$ & ${ }^{*}$ \\
\hline GPS & {$[\mathrm{m}]$} & - & ${ }^{*}$ \\
\hline Pressure & {$[\mathrm{Pa}]$} & - & ${ }^{*}$ \\
\hline Mtw Position & {$[\mathrm{cm}]$} & ${ }^{*}$ & - \\
\hline Velocity & {$[\mathrm{m} / \mathrm{s}]$} & ${ }^{*}$ & - \\
\hline 3-D Acc (BM) & {$\left[\mathrm{m} / \mathrm{s}^{2}\right]$} & ${ }^{*}$ & - \\
\hline 3-D AngVel(BM) & {$[\mathrm{rad} / \mathrm{s}]$} & ${ }^{*}$ & - \\
\hline 3-D Ang.Acc (BM) & {$\left[\mathrm{rad} / \mathrm{s}^{2}\right]$} & ${ }^{*}$ & - \\
\hline Ori (BM) & {$\left[{ }^{\circ}\right]$} & ${ }^{*}$ & - \\
\hline Joint Angle & {$\left[{ }^{\circ}\right]$} & ${ }^{*}$ & - \\
\hline Ground Truth (GT) & - & ${ }^{*}$ & ${ }^{*}$ \\
\hline
\end{tabular}

where the phone is in pocket where the most appropriate MTw revealing same properties is the one mounted on the upper leg. Similarly, we further assumed the MTw on the pelvis to simulate the scenario where the phone is being carried in backpack.

\subsubsection{Acquire Data}

The dataset containing the scenarios is defined in Table 6.3 and is available from [1]. The logged data from the phones and the MVN studio are extracted with 100 and $60 \mathrm{~Hz}$ sampling frequencies, respectively. Data from both devices are merged into a MATLAB '.mat'-file. The naming of experiments is in line with the structure given in Table 6.3. This file contains the structure of the data and attributes corresponding to each subject. For example, all measurements related to Case 4, are stored in Case 4 dataset and Case 4 . Subject (1) provides the data for all sensors and attributes associated with subject 1 .

Table 6.4 represents all available data for both devices in the measurement scenarios dataset. During the post-analysis phase of the data, some signals were identified as either corrupted or missing. As a result, the dataset contains some empty fields. The dataset contains 4 measurement scenarios with 7-8 measurement sets each, depending on the number of participants. To further simplify working with the dataset, a toy example with MATLAB code to extract the data is provided together with the dataset. The first measurement scenario (Case 1) for first subject is set as default. More details about setting variables and extracting desired outputs is provided in a README file attached to the dataset. 


\subsection{Data Analysis}

We apply a classification algorithm to the data to assign them to the defined classes in Table 6.1. This is done in two steps; feature extraction on the raw signals, followed by a classification step.

\subsubsection{Feature Extraction}

Feature extraction is a way to try to better bring out the inherent information in the available data, and reduce the dimensionality of the raw/pre-processed data in order to be able to apply classification algorithms on it.

The feature extraction phase is performed by dividing the inertial data in sliding windows of $N$ samples with no overlap. The window size must be selected such that it satisfies two different objectives. On one hand, it must be long enough to cover at least one gait cycle. On the other hand, it should be short enough to identify sudden motion mode transitions. In this work, we set the window size to 0.5 seconds. This translates to 50 and 30 samples for phone and motion trackers, respectively, imposed by their sampling frequencies.

In the rest of this section we define the features we feed to the classifier and provide more specific examples from the dataset. All given examples correspond to Case 1 described in Table 6.3 where the subject intentionally switches between the fixed and swinging device modes.

\section{Signal Norm}

For a generic signal $S[n],\|S\|_{\max }$ denotes the maximum norm over the sampling window as follows

$$
\|S\|_{\max }=\max _{n}\|S[n]\| .
$$

The norm contains useful information used to discriminate between different modes. More precisely, any change of motion mode results in a large difference in the values of accelerometer norm, $\|a\|_{\max }$. This enables the identification of a change in the motion mode. In addition, large difference in the values of the gyroscope norm $\|\omega\|_{\max }$ can be translated into a switch in the device mode.

To further illustrate the behavior of $\|a\|_{\max }$ relative to the change in the motion mode see Fig. 6.5, where the norms of signals are depicted while the smartphone is in both fixed and swinging modes. These signals correspond to Case 1 in Fig. 6.3. Fig. 6.5b further presents how the gyroscope norm, $\|\omega\|_{\max }$, assists in device mode discrimination.

\section{Signal Energy}

Let $S[n]$ be a generic signal, e.g. a single accelerometer/gyroscope direction or accelerometer/gyroscope norm. The energy of the signal $E_{S}$ is obtained by taking the squared norm of $S[n]$ and summing and normalizing it over the sliding 


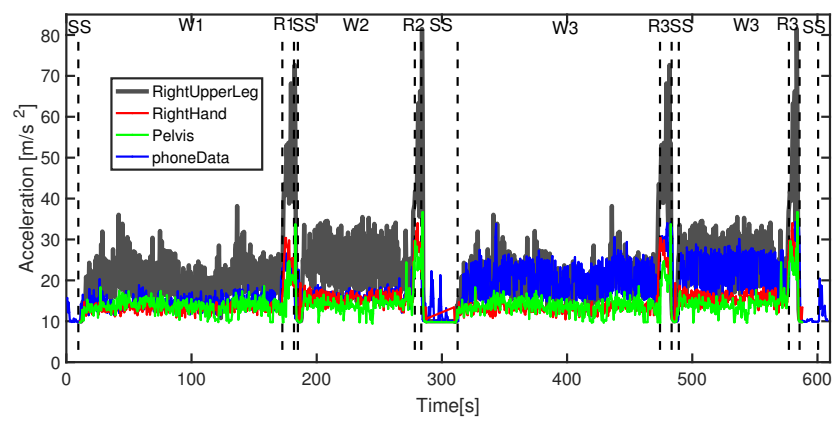

(a) Accelerometer norm of signal.

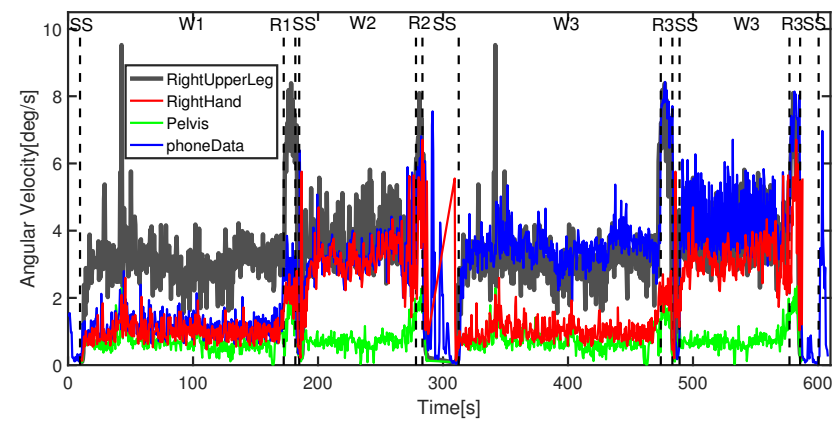

(b) Gyroscope norm of signal.

Figure 6.5: Signal norm for 4 different motion trackers together with the signal from the smartphones. The subject performed the Case 1 from Fig. 6.3. Black lines separate the different classes. Related class for each section is indicated in the figures. The subject carried a smartphone in the right hand and one in the front right pocket. The device carried by hand is switching between fixed and swinging mode [49, C2017 IEEE].

window

$$
E_{S}=\frac{1}{N} \sum_{n=0}^{N-1} S[n]^{2} .
$$

The obtained energy is a useful feature allowing us to distinguish fixed or moving device modes due to rapid response to mode transition. For example, the gyroscope will have higher energies in swinging hand device mode than the fixed hand scenario.

Fig. 6.6 shows the energy signal for both accelerometer and gyroscope. As shown in Fig. 6.6a each transition between motion modes results in a noticeable change in the energy of the accelerometer signal. The effect of the device mode on the energy signal is illustrated in Fig. 6.6b, where it is shown that switching from fixed to swinging mode increases the angular velocity energy drastically. 


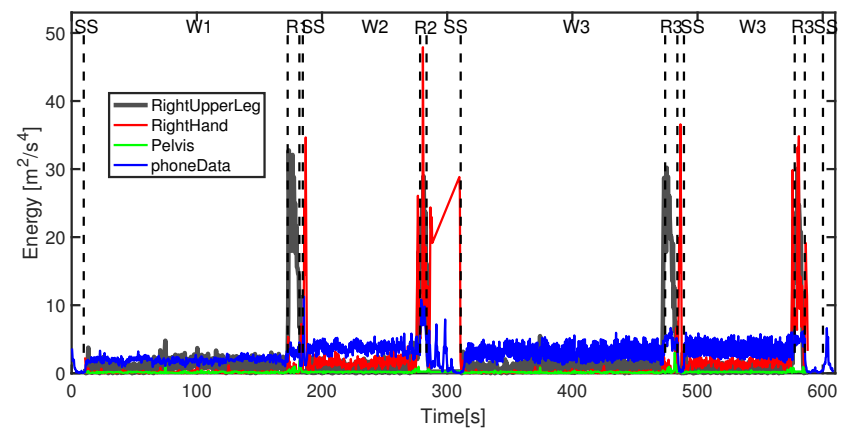

(a) Accelerometer energy signal.

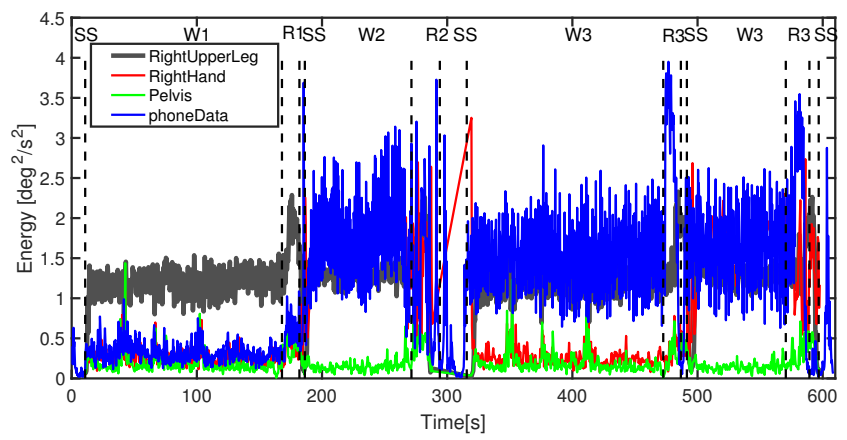

(b) Gyroscope energy signal.

Figure 6.6: Energy signal for 4 different motion trackers together with the signal from the smartphones. The sensor locations and measurement scenario are the same as the one described in Fig. 6.5 [49, (C2017 IEEE].

\section{Signal variance}

For any generic signal $S[n]$ the average of the squared differences from the mean, the variance signal, is defined as follows

$$
\sigma_{S}^{2}=\frac{1}{N-1} \sum_{n=0}^{N-1}\left(\|S[n]\|-\frac{1}{N} \sum_{n=0}^{N-1}\|S[n]\|\right)^{2} .
$$

The variance signal assists to discriminate between high and low intensity movements. For example, the estimated variance of both accelerometer and gyroscope is highly informative while the objective is to distinguish between swinging mode with any other less intense movement scenario.

Fig. 6.7 shows the signal variance of both accelerometer and gyroscope. One advantage of the variance signal is to enhance robustness of the classification algorithm in motion mode recognition. Running leads to more intense movements than both walking and standing still. Higher peaks in the signal variance, as 


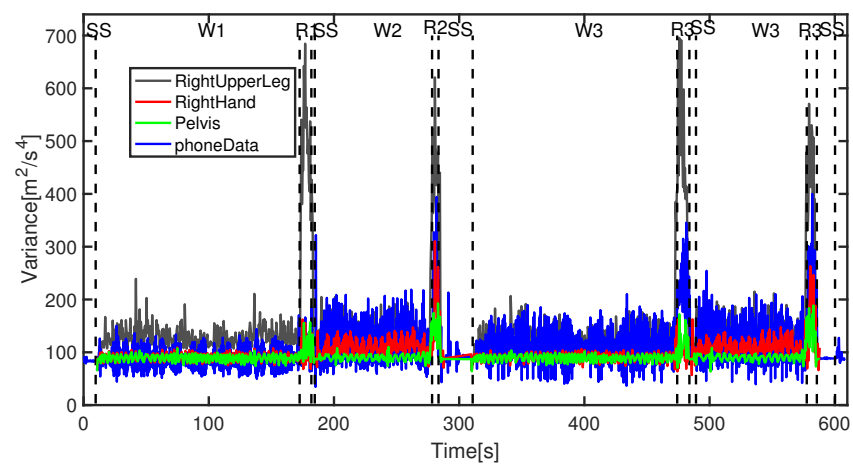

(a) Accelerometer signal variance.

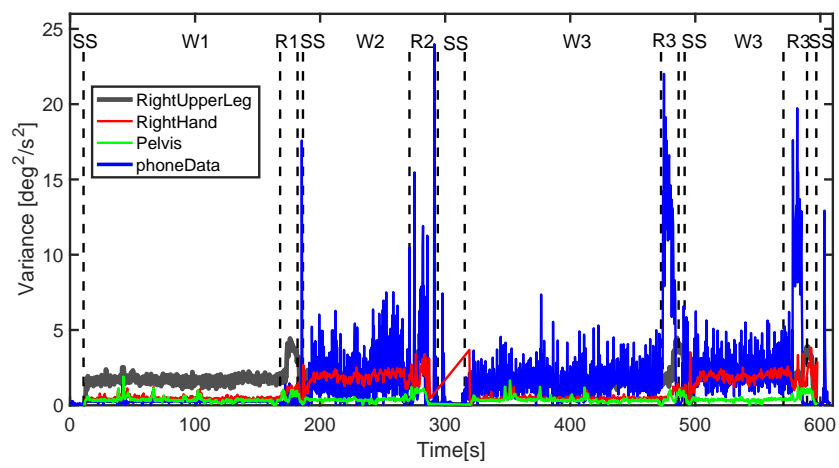

(b) Gyroscope signal variance.

Figure 6.7: Signal variance for 4 different motion trackers together with the signal from the smartphones. The sensor locations and measurement scenario are the same as the one described in Fig. 6.5 [49, (C2017 IEEE].

shown in Fig. 6.7a, can then be identified once the user switches to this mode. Additionally, the signal variance analysis can be used to recognize device mode switches. The variation of the variance signal illustrated in Fig. 6.7b shows how changing between classes with different device modes, W1 and W2 for example, can be distinguished. 


\section{Frequency Analysis}

This feature allows us to identify any movement such as periodic movement from aperiodic ones. Different activities have different frequencies, resulting in a varying power spectrum that assists in activity identification. Thus, the analysis of the frequency domain of inertial signals recorded with hand-held devices allows capturing the periodicity of the accelerometer/gyroscope signals due to the subject's activity. Presence or absence of peaks in the spectogram of the inertial signals gives useful insights whether the subject is having a periodic movement or standing still.

The spectogram of the gyroscope signal is obtained using Short Time Fourier Transform (STFT) and is reported in Fig. 6.8. The periodicity of the walking and running mode is visible in the frequency peaks of the spectogram while it is possible to identify a static case around time 300 .

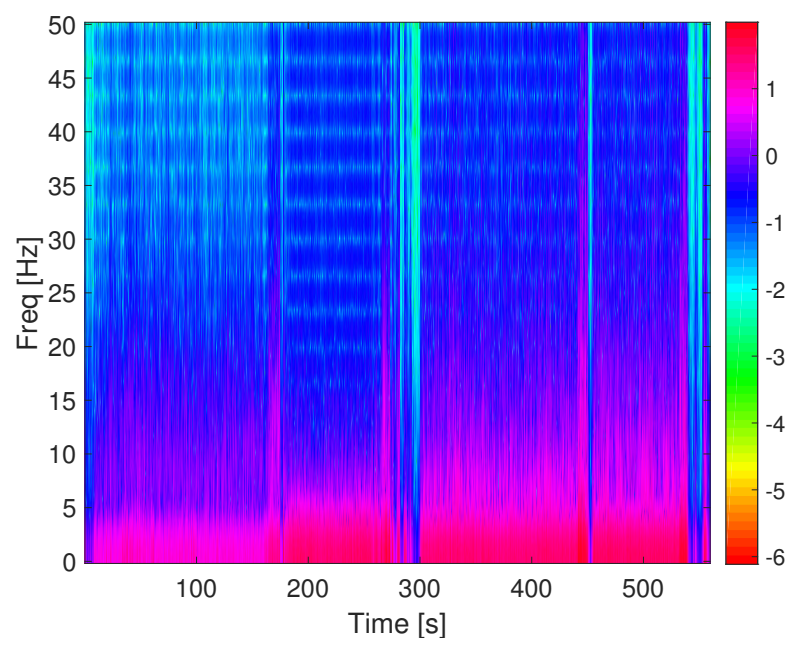

Figure 6.8: Spectogram of the smartphone's gyroscope signal carried in the right hand. The subject performed the Case 1 from Fig. 6.3 [49, (C2017 IEEE]. 


\subsubsection{Classification}

As a final step in characterizing the data, a classifier is applied to the features to try to extract the motion and device modes as defined in Table 6.1.

Different machine learning algorithms can be used for the classification. For example, [80] applies a decision tree for classification purposes while [94] uses multi-layer perceptron (MLP) and a support vector machine (SVM) to improve the performance in terms of recognition of human activity.

Two datasets, are formed for phone and motion tracker signals as described in Sec. 6.2.2, separately. Each set contains eight features constructed from four signal attributes introduced in Sec. 6.3 for both gyroscope and accelerometer. Since the classes are labeled accurately for each dataset, a supervised learning approach is applied. A multivariate decision tree classifier is trained using MATLAB; Machine learning toolbox. The classifier is validated with 10 -fold cross-validation.

The performance of the classifier for both devices are summarized in the confusion matrices given in Tables 6.5a and 6.5b. In the MTw data, having a sensor to mimic the behavior of the phone data carried by backpack is challenging. As Table 6.5a reports, the mode detection success rate for MTw sensors are over $70 \%$ for all classes. SS and W1 classes are missclassified with W4 class corresponding to the pelvis sensor. To explain this observation, one needs to note how the pelvis sensor is rigidly mounted on the body. This leads to miss-classification with other low intensity modes. With phone data, the classification results are promising as represented by Table $6.5 \mathrm{~b}$. All the classes are detected correctly with over $75 \%$ for walking and standing still modes and over $56 \%$ for running mode. Running mode recognition is challenging as the phone has movement. Missclassification of R1 with R4 and W1 with W4 can be explained by the fact that the phone is fixed in either of these situations. 
Table 6.5: Confusion matrix of the modes classification represents in Table 6.1 using the binary decision tree classifier using cross-validation with 10 folds. The table shows how different annotated activities are classified in [\%] [49, O2017 IEEE].

(a) Data from 4 different motion trackers

Recognised activity

\begin{tabular}{|c|c|c|c|c|c|c|c|c|c|}
\hline & \& & $R^{2}$ & $P^{3}$ & $R^{*}$ & ss & $s$ & $\Delta^{2}$ & $N^{3}$ & $s^{\star}$ \\
\hline R1 & 83 & 8 & 0 & 6 & 0 & 0 & 0 & 0 & 0 \\
\hline $\mathrm{R} 2$ & 5 & 72 & 1 & 0 & 0 & 0 & 0 & 1 & 0 \\
\hline R3 & 0 & 2 & 96 & 0 & 0 & 0 & 0 & 0 & 0 \\
\hline R4 & 5 & 0 & 0 & 85 & 1 & 0 & 0 & 0 & 1 \\
\hline SS & 0 & 2 & 0 & 1 & 79 & 2 & 0 & 0 & 1 \\
\hline W1 & 0 & 0 & 1 & 1 & 5 & 78 & 1 & 0 & 13 \\
\hline W2 & 0 & 8 & 0 & 0 & 0 & 1 & 84 & 6 & 0 \\
\hline W3 & 7 & 8 & 1 & 3 & 2 & 1 & 13 & 92 & 0 \\
\hline W4 & 0 & 0 & 1 & 4 & 13 & 17 & 1 & 0 & 84 \\
\hline
\end{tabular}

(b) Data from phones.

Recognised activity

\begin{tabular}{|c|c|c|c|c|c|c|c|c|c|}
\hline & 2 & $\mathrm{P}^{2}$ & $p^{3}$ & $P^{*}$ & ss & $\Delta$ & $\Delta^{2}$ & $\$^{3}$ & $s^{*}$ \\
\hline R1 & 58 & 3 & 0 & 0 & 0 & 0 & 0 & 2 & 0 \\
\hline R2 & 1 & 60 & 4 & 4 & 0 & 0 & 1 & 2 & 0 \\
\hline R3 & 0 & 13 & 83 & 0 & 1 & 0 & 0 & 0 & 0 \\
\hline $\mathrm{R} 4$ & 20 & 3 & 0 & 56 & 0 & 0 & 0 & 2 & 0 \\
\hline SS & 1 & 0 & 6 & 0 & 84 & 4 & 3 & 2 & 1 \\
\hline W1 & 3 & 0 & 0 & 7 & 7 & 83 & 3 & 1 & 8 \\
\hline W2 & 1 & 3 & 6 & 7 & 2 & 1 & 77 & 14 & 0 \\
\hline W3 & 14 & 17 & 2 & 22 & 1 & 1 & 16 & 75 & 0 \\
\hline W4 & 0 & 0 & 0 & 4 & 5 & 11 & 1 & 0 & 90 \\
\hline
\end{tabular}


Part III

\section{Conclusion and Future works}





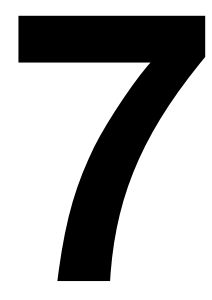

\section{Concluding Remarks}

Mobile positioning applications are enabled by using measurements provided by almost all recent smartphones. In this thesis, we contribute to the development of models for different measurements and investigate how their parameters can be estimated. Given that the parameters are known, the position of the mobile user can be estimated. The position of a mobile device can be inferred from models relating the measurements provided by sensors and receivers equipped in the measuring device to its position. The positioning performance depends on the quality of measurements on one hand and the accuracy of the models on the other.

In this thesis, we investigated how to estimate unknown parameters of different models. The measurements considered here can be provided by almost all recent smartphones. Among different alternatives, we consider radio-based RSS, GPS, and inertial measurements. Unknown parameters of the models are then estimated using a least square estimator or in a filtering framework.

To address the reliability issue of GNSS methods, while taking advantage of their accuracy, they were used upon availability of strong satellite signals. For instance, positioned RSS signals are used to estimate the unknown parameters of the RSS model in an offline phase. The estimated parameters can then be used in RSS-based positioning methods. Furthermore, a gait model is developed in which the gait parameters and the position of the mobile users are estimated using inertial sensor measurements. However, solely relying on the inertial sensors will lead to cumulative error in the estimations. To address this, in time intervals when GPS signals are available, we use them to correct our estimates.

The performance of the developed methods are evaluated on simulated as well as real data and the results are presented. In the rest of this section, we first provide concluding remarks in each application and then finalize the work with some future research directions. 


\subsection{Antenna gait parameters estimation}

The semi-empirical joint antenna and propagation model parameter estimation was described in Section 4.2. The associated parameter estimation problem can be formulated as a least square problem, which enables efficient estimation of the model parameters in Section 2.1.3. The developed model accounts for both the RSS decay rate using an empirical log-distance and the deterministic antenna radiation models. The performance of the proposed method was evaluated using both simulation data and real RSS measurements. The results indicated that the joint antenna and propagation model provide significantly better accuracy compared to a propagation model alone as reported in Section 4.3.

\subsection{Pedestrian dead reckoning positioning}

Inertial sensors measurements are another source of information that can be used either in standalone positioning algorithms or together with other radio measurements. To mitigate the main disadvantage of the standalone INS systems, being the integration drift caused by integrating inertial sensors measurements, a framework for a pedestrian dead reckoning algorithm was developed. In this application, we proposed two extended PDR models in Section 5.1.1 which were the extended version of basic PDR, given in Section 3.3. The approach was based on an offline and online multi-rate Kalman filter bank, given in Section 5.3, that learned gait parameters in time intervals when GPS position estimates were available. This improved the PDR in time intervals when the measurement is unavailable. The proposed methods had been evaluated using simulated data as well as real experiment of IMU measurements, given in Section 5.4 where it was shown that promising results could be obtained especially when the step detection threshold was chosen adaptively.

In the offline approach, the algorithm assumed a subject moving with constant speed. It is explained that the results deteriorate if a fixed threshold is used that is not appropriately chosen to suit the current user. Given the experimental data provided in this work a low step detection threshold would probably work satisfactory.

To relax the constant velocity assumption, the online approach set no restriction on the subjects speed and the experiment was done while different walking models were considered; running, walking and standing still. However, it was shown in the online Kalman filter bank in Section 5.4.2 that by having more variations in the user's walking patterns and with regular stops or running, the step detection and position estimation accuracy were affected.

\subsection{Motion and Device Mode Classification}

To overcome the negative effect of the multiple walking patterns and also to extend the algorithm to more realistic scenarios, the user's motion modes need to be known. An extensive dataset was presented for classification and investigation 
of the motion and device mode for pedestrian navigation systems, where many device modes, a number of users and a few motion modes were logged for three different trajectories. All the experiment setup and details about the dataset were given in Section 6.1 and Section 6.2, respectively. All data were fully annotated with ground truth classes, and other metadata such as position and accurate full body motion using bio-mechanical models.

It was discussed how the motion and device mode relate to pedestrian deadreckoning algorithms, the working horse of PNS, and how PNS can otherwise benefit from improved and extended mode classification in Section 6.3. The larger part of the work described the field tests in detail. The last part, Section 6.3.2, suggested a few features that can be computed from the logged data, and applied a straight forward classifier on these features. The result was promising, yet preliminary. The dataset is publicly available [1], and we hope the research community can benefit from this to improve the classification results further.

\subsection{Future Work}

A future extension of this work can be to combine the algorithms developed in this thesis. This allows for a robust localization algorithm operating in outdoor and indoor environments with and without GPS signals. Using positioned RSS measurements is an example of how to make the algorithm robust against GPS denied signal conditions. Classification methods could also be efficiently combined with the proposed gait parameter estimation algorithm to give a more accurate step detection while the user's motion mode varies along the trajectory. In a real life navigation session the user's motion can be a combination of walking, running, and standing still. Knowledge of the motion status of the user drastically improves the gait parameter estimations leading to a more accurate positioning. Classification methods, as we discussed in Chapter 6, allow for distinguishing between the motion modes that can be added to the algorithm as a pre-process, prior to the step detection phase. Thus, one interesting idea is to combine the motion mode and device mode information with PDR positioning application to improve, in an online fashion, the step detection and step length estimation algorithms. 



\section{Bibliography}

[1] IMU Dataset for Device and Motion Mode Classification. URL http:// users.isy.liu.se/rt/parka23/research.html. Cited on pages 81 and 93.

[2] Senion AB. Senion, 2017. URL https://goo.gl/NjVkk2. Cited on page 5.

[3] M.A. Al-Ammar, S. Alhadhrami, A. Al-Salman, A. Alarifi, H.S. Al-Khalifa, A. Alnafessah, and M. Alsaleh. Comparative survey of indoor positioning technologies, techniques, and algorithms. In International Conference on Cyberworlds (CW), Santander, Spain, October 2014. Cited on page 23.

[4] J. C. Alvarez, D. Alvarez, A. López, and R. C. González. Pedestrian navigation based on a waist-worn inertial sensor. Sensors, 12(8):10536-10549, 2012. Cited on pages 4 and 5.

[5] B.D. Anderson and J.B. Moore. Optimal Filtering. Prentice Hall, Inc, 1979. ISBN 0-13-638122-7. Cited on pages 19 and 20.

[6] P. Andersson. Adaptive forgetting in recursive identification through multiple models. Analysis and Optimization of Systems, 62:171-185, 1984. Cited on page 54 .

[7] K. J. Åström. Introduction to Stochastic Control Theory. Dover Publications, Mineola, NY, USA, 2006. Cited on page 15.

[8] Paramvir Bahl and Venkata N. Padmanabhan. RADAR: an in-building RFbased user location and tracking system. In Institute of Electrical and Electronics Engineers, Inc., March 2000. Cited on page 3.

[9] Y. Bar-Shalom, X. R. Li, and T. Kirubarajan. Estimation with Applications to Tracking and Navigation. Wiley-Interscience, 2001. Cited on pages 11, 15, 18,57 , and 58 .

[10] Y. Bar-Shalom, S. Challa, and H. A. P. Blom. IMM estimator versus optimal estimator for hybrid systems. IEEE Trans. Aerosp. Electron. Syst., 41(3):986991, July 2005. Cited on page 57. 
[11] S. Beauregard. Omnidirectional pedestrian navigation for first responders. In 4th Workshop on Positioning, Navigation and Communication (WPNC), Hannover, Germany, March 2007. Cited on pages 4 and 5.

[12] H.A.P Blom and Y. Bar-Shalom. The interacting multiple model algorithm for systems with markovian switching coefficients. IEEE Transactions on Automatic Control, 33(8):780-783, 1988. Cited on page 54.

[13] C. Botteron, M. Fattouche, and A. Host-Madsen. Statistical theory of the effects of radio location system design parameters on the positioning performance. In IEEE 56th Vehicular Technology Conference, (VTC 2002-Fall), volume 2, pages 1187-1191, 2002. doi: 10.1109/VETECF.2002.1040792. Cited on page 3 .

[14] James J. Caffery. Wireless Location in CDMA Cellular Radio Systems. Kluwer Academic Publishers, Norwell, MA, USA, 1999. ISBN 0792377036. Cited on pages 2 and 36.

[15] Jonas Callmer. Autonomous Localization in Unknown Environments. PhD thesis, Linköping University, 2013. Cited on page 27.

[16] N. Chang, R. Rashidzadeh, and M. Ahmadi. Robust indoor positioning using differential Wi-Fi access points. Consumer Electronics, IEEE Transactions on, 56(3):1860-1867, Aug 2010. ISSN 0098-3063. doi: 10.1109/TCE.2010. 5606338. Cited on page 2 .

[17] Aaron J. Coutts and Rob Duffield. Validity and reliability of GPS devices for measuring movement demands of team sports. Journal of Science and Medicine in Sport, 13(1):133 - 135, 2010. ISSN 1440-2440. doi: http://dx. doi.org/10.1016/j.jsams.2008.09.015. Cited on page 2 .

[18] E. Damasso and L. M. Correia, editors. Digital mobile radio towards future generation systems. European Cooperation in the field of Scientific and Technical research (COST), Brussels, Belgium, 1999. Cited on page 36.

[19] M. Eladio, V. Oriol, F. Gerald, and R. Ruzena. Precise indoor localization using smart phones. In Proceedings of the International Conference on Multimedia, MM '10, pages 787-790, New York, NY, USA, 2010. ACM. ISBN 978-1-60558-933-6. doi: 10.1145/1873951.1874078. Cited on page 2.

[20] M. Elhoushi, J. Georgy, A. Noureldin, and M.J Korenberg. Motion mode recognition for indoor pedestrian navigation using portable devices. IEEE Transactions on Instrumentation and Measurement, 65(1):208-221, 2016. Cited on page 6 .

[21] M. Elhoushi, J. Georgy, A. Noureldin, and M.J Korenberg. A survey on approaches of motion mode recognition using sensors. IEEE Transactions on Intelligent Transportation Systems, PP(99):1-25, 2016. Cited on page 6. 
[22] R. Faragher and R. Harle. An analysis of the accuracy of bluetooth low energy for indoor positioning applications. In Proceedings of the 27th International Technical Meeting of The Satellite Division of the Institute of Navigation (ION GNSS+ 2014), Tampa, Florida, September 2014. Cited on page 32.

[23] Z. Farid, R. Nordin, and M. Ismail. Recent advances in wireless indoor localization techniques and system. Journal of Computer Networks and Communications, 2013(15):1-12, August 2013. Cited on page 31.

[24] C. Feng, W.S.A. Au, S. Valaee, and T. Zhenhui. Received-Signal-StrengthBased indoor positioning using compressive sensing. IEEE Transactions on Mobile Computing, 11(12):1983-1993, Dec 2012. ISSN 1536-1233. doi: 10.1109/TMC.2011.216. Cited on page 2.

[25] Y. Feng, Y. Zhao, and F. Gunnarsson. Proximity report triggering threshold optimization for network-based indoor positioning. In 18th International Conference on Information Fusion (Fusion), Washington, DC, USA, July 2015. Cited on page 32.

[26] E. Foxlin. Pedestrian tracking with shoe-mounted inertial sensors. IEEE Comput. Graph., 25:38-46, 2005. Cited on page 5.

[27] S. Garcia-Villalonga and A. Perez-Navarro. Influence of human absorption of Wi-Fi signal in indoor positioning with Wi-Fi fingerprinting. In International Conference on Indoor Positioning and Indoor Navigation (IPIN), Banff, AB, Canada, October 2015. Cited on page 31.

[28] GIS Geography. World geodetic system WGS84, 2017. URL https : / / goo. gl/BgGVV7. Cited on page 29.

[29] GPS.Gov. Official U.S. government information about the global positioning system (GPS) and related topics, 2017. URL https://goo.gl/t6PxbU. Cited on page 29.

[30] F. Gunnarsson, M.N. Johansson, A. Furuskar, M. Lundevall, A. Simonsson, C. Tidestav, and M. Blomgren. Downtilted base station antennas - a simulation model proposal and impact on HSPA and LTE performance. In IEEE 68th Vehicular Technology Conference (VTC 2008-Fall), pages 1-5, Sept 2008. doi: 10.1109/VETECF.2008.49. Cited on page 37.

[31] F. Gunnarsson, F. Gunnarsson and N. Carlsson. Particle filtering for networkbased positioning terrestrial radio networks. In IET Conference on Data Fusion Target Tracking: Algorithms and Applications (DF TT 2014), pages 1-7, April 2014. doi: 10.1049/cp.2014.0523. Cited on page 3.

[32] C. Gusenbauer, D. Isert and J. Krosche. Self-contained indoor positioning on off-the-shelf mobile devices. In Proceedings of IEEE 2nd Conference on Indoor Positioning and Indoor Navigation, Zurich, Switzerland, September 2010. Cited on page 5. 
[33] F. Gustafsson. Statistical Sensor Fusion. Studentlitteratur, 2010. Cited on pages 48 and 55 .

[34] F. Gustafsson and F. Gunnarsson. Mobile positioning using wireless networks: possibilities and fundamental limitations based on available wireless network measurements. IEEE Signal Processing Magazine, 22(4):41-53, July 2005. ISSN 1053-5888. doi: 10.1109/MSP.2005.1458284. Cited on pages 2, 3 , and 44 .

[35] F. Gustafsson, F. Gunnarsson, N. Bergman, and U. Forssell. Particle filters for positioning, navigation and tracking. IEEE Transactions on Signal Processing, 50(2):425-437, February 2002. Cited on page 27.

[36] M. Hata. Empirical formula for propagation loss in land mobile radio services. IEEE J VT, 29(3):317-325, 1980. Cited on page 36.

[37] G. Hendeby. Performance and Implementation Aspects of Nonlinear Filtering. PhD thesis, Linköping Studies in Science and Technology., 2008. Cited on page 54 .

[38] G. Hendeby, F. Gustafsson, and N. Wahlström. Teaching sensor fusion and Kalman filtering using a smartphone. In Proceedings of the 19th IFAC World Congress, Cape Town, South Africa, August 2014. Cited on page 63.

[39] G. Hendeby, F. Gustafsson, N. Wahlström, and S. Gunnarsson. Platform for teaching sensor fusion using a smartphone. International journal of engineering education, 33(2(B)):781-789, 2017. Cited on pages 42 and 75 .

[40] N. Ho, P. Truong, and G. Jeong. Step-detection and adaptive step-length estimation for pedestrian dead-reckoning at various walking speeds using a smartphone. Sensors, 16(9):1-13, September 2016. Cited on pages 50, 52, and 53.

[41] A. H. Jazwinski. Stochastic processes and filtering theory, volume 64. Academic Press, Inc., 1970. Cited on page 21.

[42] R. Jirawimut, P. Ptasinski, V. Garaj, F. Cecelja, and W. Balachandran. A method for dead reckoning parameter correction in pedestrian navigation system. IEEE Trans. Instrum. Meas., 52:209-215, 2003. ISSN 0018-9456. Cited on page 4 .

[43] T. Kailath, A.H. Sayed, and B. Hassibi. Linear Estimation. Prentice Hall, 2000. ISBN 978-0-13-022464-4. Cited on pages 19 and 20.

[44] R.E. Kalman. New approach to linear filtering and prediction problems. Transactions of the ASME-Journal of Basic Engineering, 82:35-45, 1960. Cited on page 19. 
[45] W. Kang and Y. Han. SmartPDR: Smartphone-based pedestrian dead reckoning for indoor localization,". IEEE Sensors Journal, 15(6):2906-2916, May 2015. Cited on pages 49, 50, and 52.

[46] P. Kasebzadeh, G.-S. Granados, and E.S. Lohan. Indoor localization via WLAN path-loss models and Dempster-Shafer combining. In International Conference on Localization and GNSS (ICL-GNSS), pages 1-6, June 2014. doi: 10.1109/ICL-GNSS.2014.6934173. Cited on pages 2, 30, and 36.

[47] P. Kasebzadeh, C. Fritsche, E. Özkan, F. Gunnarsson, and F. Gustafsson. Joint antenna and propagation model parameter estimation using rss measurements. In 18th International Conference on Information Fusion (Fusion), Washington, DC, USA, July 2015. Cited on pages 2, 35, 38, 40, 41, 42, 43, and 44 .

[48] P. Kasebzadeh, C. Fritsche, G. Hendeby, F. Gunnarsson, and F. Gustafsson. Improved pedestrian dead reckoning positioning with gait parameter learning. In International Conference on Information Fusion, Heidelberg, Germany, July 2016. Cited on pages 2, 5, 45, 46, 47, 51, 52, 54, 55, 56, 63, 64, and 65.

[49] P. Kasebzadeh, G. Hendeby, C. Fritsche, F. Gunnarsson, and F. Gustafsson. Imu dataset for motion and device mode classification. In 8th internation Conference on Indoor Positioning and Indoor Navigation (IPIN2017), Sapporo, Japan, September 2017. Cited on pages 2, 7, 73, 74, 75, 76, 77, 79, 80, $81,83,84,85,86$, and 88 .

[50] S.M. Kay. Fundamentals of Statistical Signal Processing: Estimation Theory, volume 1. Prentice-Hall, Inc, 1993. ISBN 0-13-042268-1. Cited on page 11.

[51] J. W. Kim and D. H. Jang, J. J .and Hwang. A step, stride and heading determination for the pedestrian navigation system. Journal of Global Positioning Systems, 3(1-2):273-279, 2004. Cited on page 4.

[52] M. Kok. Probabilistic modeling for sensor fusion with inertial measurements. PhD thesis, Linköping studies in science and technology, 2017. Cited on pages 24,25 , and 28 .

[53] F. Lassabe, P. Canalda, P. Chatonnay, and F. Spies. Indoor Wi-Fi positioning: techniques and systems. annals of telecommunications - annales des télécommunications, 64(9-10):651-664, 2009. ISSN 0003-4347. doi: 10.1007/s12243-009-0122-1. Cited on page 2.

[54] E.L. Lehmann. Theory of Point Estimation. Probability and Mathematical Statistics. John Wiley \& Sons, Ltd, 1983. ISBN 0-471-05849-1. Cited on page 11 .

[55] R.W. Levi and T. Judd. Dead reckoning navigational system using accelerometer to measure foot impacts, 1996. Cited on page 4. 
[56] J.F. Li, Q.H. Wang, X.M. Liu, and M.Y. Zhang. An autonomous waistmounted pedestrian dead reckoning system by coupling low-cost MEMS inertial sensors and GPS receiver for 3D urban navigation. Journal of Engineering Science and Technology, 7(9):9-14, 2014. Cited on pages 4 and 5.

[57] Linköping University, Sweden. Sensor fusion app, December 2014. URL https://goo.gl/0qNyu. Cited on pages 63 and 75.

[58] Y. Liu, Y. Chen, L. Shi, Z. Tian, M. Zhou, and L. Li. Accelerometer based joint step detection and adaptive step length estimation algorithm using handheld devices. Journal of Communications, 10(7):520-525, 2015. Cited on pages $4,5,49,50$, and 52 .

[59] L. Ljung. System Identification: Theory for the User. Prentice Hall, Upper Saddle River, NJ, USA, 1999. ISBN 978-0-13-656695-3. Cited on page 15.

[60] H. J. Luinge and P.H. Veltink. Inclination measurement of human movement using a 3-D accelerometer with autocalibration. IEEE Transactions Neural System Rehabil. Eng., 12(1):112-121, Mar. 2004. Cited on pages 4 and 5.

[61] J.T. MacDonald, D.A. Roberson, and D.R. Ucci. Location estimation of isotropic transmitters in wireless sensor networks. In IEEE Military Communications Conference (MILCOM), pages 1-5, Oct 2006. doi: 10.1109/ MILCOM.2006.302347. Cited on page 3.

[62] MapToaster. How GPS works, 2017. URL https://goo.gl/khHmxw. Cited on page 29.

[63] Medium. Recently popular techniques and technologies used for indoor locationing systems, 2017. URL https://goo.gl/389f69. Cited on page 31.

[64] R. S. Nerem and K. M. Larson. Global positioning system, theory and practice, 5th edition. Eos, Transactions American Geophysical Union, 82(33): 365-365, 2001. ISSN 2324-9250. doi: 10.1029/01EO00224. Cited on page 2.

[65] N. Patwari, J.N. Ash, S. Kyperountas, A.O. Hero, R.L. Moses, and N.S. Correal. Locating the nodes: cooperative localization in wireless sensor networks. IEEE Signal Processing Magazine, 22(4):54-69, July 2005. ISSN 1053-5888. doi: 10.1109/MSP.2005.1458287. Cited on page 3.

[66] L. Pei, J. Liu, R. Guinness, Y. Chen, H. Kuusniemi, and R. Chen. Using LSSVM based motion recognition for smartphone indoor wireless positioning. Sensors, 12:6155-6175, 2012. Cited on page 6.

[67] K. Radnosrati, F. Gunnarsson, and F. Gustafsson. New trends in radio network positionig. In 18th International Conference on Information Fusion (Fusion), Washington, DC, USA, July 2015. Cited on page 2. 
[68] K. Radnosrati, C. Fritsche, G. Hendeby, F. Gunnarsson, and F. Gustafsson. Fusion of TOF and TDOA for 3GPP positioning. In 19th International Conference on Information Fusion (FUSION), Heidelberg, Germany, July 2016. Cited on page 2 .

[69] T.S. Rappaport. Wireless Communications: Principles and Practice. Prentice Hall PTR, Upper Saddle River, NJ, USA, 2nd edition, 2001. ISBN 0130422320. Cited on pages 29, 30, and 36.

[70] A. Reiss, G. Hendeby, and D. Stricker. A competitive approach for human activity recognition on smartphones. In European Symposium on Artificial Neural Networks, Computational Intelligence and Machine Learning (ESANN), Bruges, Belgium, April 2013. Cited on page 6.

[71] V. Renaudin, M. Susi, and G. Lachapelle. Step length estimation using handheld inertial sensors. IEEE Sensors Journal, 12:8507-8525, 2012. Cited on pages $46,49,50$, and 53 .

[72] V. Renaudin, V. Demeule, and M. Ortiz. Adaptative pedestrian displacement estimation with a smartphone. In International Conference on Indoor Positioning and Indoor Navigation (IPIN), Calgary, Canada, October 2013. Cited on page 46.

[73] S. Särkkä. Bayesian Filtering and Smoothing. Cambridge University Press, 2013. Cited on page 18 .

[74] Stanley F. Schmidt. Applications of state space methods to navigation problems. in C. T. Leondes, Editor, Advanced Control Systems, 3:293-340, 1966. Cited on page 21 .

[75] B. Shin, S. Lee, C. Kim, J. Kim, T. Lee, C. Kee, S. Heo, and H. Rhee. Implementation and performance analysis of smartphone-based 3D pdr system with hybrid motion and heading classifier. In Position, Location and Navigation Symposium (PLANS), July 2014. Cited on page 49.

[76] B. Shin, C. C. Kim, J. Kim, S. Lee, C. Kee, H. Kim, and T. Lee. Motion recognition-based 3D pedestrian navigation system using smartphone. IEEE Sensors Journal, 16(18):6977-6989, September 2016. Cited on pages 49 and 50 .

[77] S. H. Shin, C. G. Park, J. W. Kim, H. S. Hong, and J. M. Lee. Adaptive step length estimation algorithm using low-cost MEMS inertial sensors. In Sensors Applications Symposium (SAS '07), San Diego, California, USA, February 2007. Cited on page 4 .

[78] Gerald L. Smith, Stanley F. Schmidt, and Leonard A. McGee. Application of statistical filter theory to the optimal estimation of position and velocity on board a circumlunar vehicle. Technical report, NASA TR R-135, National Aeronatics and Space Administration, 1962. Cited on page 21. 
[79] U. Steinhoff and B. Schiele. Dead reckoning from the pocket-an experimental study. In IEEE International Conference on Pervasive Computing and Communications (PerCom), Mannheim, Germany, March 2010. Cited on page 5.

[80] V. Susi, M. Renaudin and G. Lachapelle. Motion mode recognition and step detection algorithms for mobile phone users. Sensors, 13(2):1539-1562, 2013. Cited on pages 6 and 87 .

[81] P. Tarrío, Ana M. Bernardos, and J.R. Casar. Weighted least squares techniques for improved Received Signal Strength Based localization. Sensors, 11(9):8569-8592, 2011. ISSN 1424-8220. doi: 10.3390/s110908569. Cited on page 3 .

[82] Q. Tian, K.I-K. Salcic, Z. Wang, and Y. Pan. A multi-mode dead reckoning system for pedestrian tracking using smartphones. IEEE Sensors Journal, 16 (7):2079 - 2093, 2016. Cited on page 6 .

[83] Z. Tian, Y. Zhang, M. Zhou, and Y. Liu. Pedestrian dead reckoning for MARG navigation using a smartphone. EURASIP Journal on Advances in Signal Processing, 16(1):2-11, 2014. Cited on pages 4, 5, 49, and 52.

[84] D.H. Titterton and J.L. Weston. Strapdown inertial navigation technology. Peter Peregrinis Ltd. on behalf of the Institution of Electrical Engineers, 1997. Cited on pages 25 and 26.

[85] S. Tomažič and I. Škrjanc. Fusion of visual odometry and inertial navigation system on a smartphone. Elsevier on Computers in Industry, 74:119-134, 2015. Cited on pages 4 and 5 .

[86] Target Tracking. Derivation of the IMM filter, 2014. URL https://goo. gl/MHyNuG. Cited on page 58.

[87] Q.D. Vo and P. De. A survey of fingerprint-based outdoor localization. IEEE Communication Surveys and Tutorials, 18(1):491-506, Firstquarter 2016. Cited on page 30 .

[88] O.J. Woodman. An introduction to inertial navigation. Technical Report 696, University Of Cambridge, 2007. Cited on page 46.

[89] S. Xia, Y. Liu, G. Yuan, M. Zhu, and Z. Wang. Indoor fingerprint positioning based on wi-fi: An overview. ISPRS International Journal of GeoInformation, 6(5), April 2017. Cited on page 31.

[90] Z. Yang, C. Wu, and Y. Liu. Locating in fingerprint space: Wireless Indoor Localization with little human intervention. In Proceedings of the 18th Annual International Conference on Mobile Computing and Networking, Mobicom '12, pages 269-280, New York, NY, USA, 2012. ACM. ISBN 978-14503-1159-5. doi: 10.1145/2348543.2348578. Cited on page 2. 
[91] Jaegeol Yim, Chansik Park, Jaehun Joo, and Seunghwan Jeong. Extended Kalman filter for wireless LAN based indoor positioning. Decision Support Systems, 45(4):960 - 971, 2008. ISSN 0167-9236. doi: http://dx.doi. org/10.1016/j.dss.2008.03.004. Information Technology and Systems in the Internet-Era. Cited on page 2.

[92] Jie Yin, Qiang Yang, and L.M. Ni. Learning adaptive temporal radio maps for signal-strength-based location estimation. IEEE Transactions on Mobile Computing, 7(7):869-883, July 2008. ISSN 1536-1233. doi: 10.1109/TMC. 2007.70764. Cited on page 3 .

[93] Paul A Zandbergen. Accuracy of iPhone locations: A comparison of assisted GPS, WiFi and cellular positioning. Transactions in GIS, 13:5-25, 2009. ISSN 1467-9671. doi: 10.1111/j.1467-9671.2009.01152.x. Cited on page 2.

[94] H. Zhang, W. Yuan, Q. Shen, T. Li, and H. Chang. A handheld inertial pedestrian navigation system with accurate step modes and device poses recognition. IEEE Sensors Journal, 15(3):1421-1429, 2015. Cited on pages 4, 5, 6, 50,53 , and 87. 



\section{Licentiate Theses \\ Division of Automatic Control \\ Linköping University}

P. Andersson: Adaptive Forgetting through Multiple Models and Adaptive Control of Car Dynamics. Thesis No. 15, 1983.

B. Wahlberg: On Model Simplification in System Identification. Thesis No. 47, 1985.

A. Isaksson: Identification of Time Varying Systems and Applications of System Identification to Signal Processing. Thesis No. 75, 1986.

G. Malmberg: A Study of Adaptive Control Missiles. Thesis No. 76, 1986.

S. Gunnarsson: On the Mean Square Error of Transfer Function Estimates with Applications to Control. Thesis No. 90, 1986.

M. Viberg: On the Adaptive Array Problem. Thesis No. 117, 1987.

K. Ståhl: On the Frequency Domain Analysis of Nonlinear Systems. Thesis No. 137, 1988.

A. Skeppstedt: Construction of Composite Models from Large Data-Sets. Thesis No. 149, 1988.

P. A. J. Nagy: MaMiS: A Programming Environment for Numeric/Symbolic Data Processing. Thesis No. 153, 1988.

K. Forsman: Applications of Constructive Algebra to Control Problems. Thesis No. 231, 1990.

I. Klein: Planning for a Class of Sequential Control Problems. Thesis No. 234, 1990.

F. Gustafsson: Optimal Segmentation of Linear Regression Parameters. Thesis No. 246, 1990.

H. Hjalmarsson: On Estimation of Model Quality in System Identification. Thesis No. 251, 1990.

S. Andersson: Sensor Array Processing; Application to Mobile Communication Systems and Dimension Reduction. Thesis No. 255, 1990.

K. Wang Chen: Observability and Invertibility of Nonlinear Systems: A Differential Algebraic Approach. Thesis No. 282, 1991.

J. Sjöberg: Regularization Issues in Neural Network Models of Dynamical Systems. Thesis No. 366, 1993.

P. Pucar: Segmentation of Laser Range Radar Images Using Hidden Markov Field Models. Thesis No. 403, 1993.

H. Fortell: Volterra and Algebraic Approaches to the Zero Dynamics. Thesis No. 438, 1994.

T. McKelvey: On State-Space Models in System Identification. Thesis No. 447, 1994.

T. Andersson: Concepts and Algorithms for Non-Linear System Identifiability. Thesis No. 448, 1994.

P. Lindskog: Algorithms and Tools for System Identification Using Prior Knowledge. Thesis No. 456, 1994.

J. Plantin: Algebraic Methods for Verification and Control of Discrete Event Dynamic Systems. Thesis No. 501, 1995.

J. Gunnarsson: On Modeling of Discrete Event Dynamic Systems, Using Symbolic Algebraic Methods. Thesis No. 502, 1995.

A. Ericsson: Fast Power Control to Counteract Rayleigh Fading in Cellular Radio Systems. Thesis No. 527, 1995.

M. Jirstrand: Algebraic Methods for Modeling and Design in Control. Thesis No. 540, 1996.

K. Edström: Simulation of Mode Switching Systems Using Switched Bond Graphs. Thesis No. 586, 1996. 
J. Palmqvist: On Integrity Monitoring of Integrated Navigation Systems. Thesis No. 600, 1997.

A. Stenman: Just-in-Time Models with Applications to Dynamical Systems. Thesis No. 601, 1997.

M. Andersson: Experimental Design and Updating of Finite Element Models. Thesis No. 611, 1997.

U. Forssell: Properties and Usage of Closed-Loop Identification Methods. Thesis No. 641, 1997.

M. Larsson: On Modeling and Diagnosis of Discrete Event Dynamic systems. Thesis No. 648, 1997.

N. Bergman: Bayesian Inference in Terrain Navigation. Thesis No. 649, 1997.

V. Einarsson: On Verification of Switched Systems Using Abstractions. Thesis No. 705, 1998.

J. Blom, F. Gunnarsson: Power Control in Cellular Radio Systems. Thesis No. 706, 1998.

P. Spångéus: Hybrid Control using LP and LMI methods - Some Applications. Thesis No. 724, 1998.

M. Norrlöf: On Analysis and Implementation of Iterative Learning Control. Thesis No. 727, 1998.

A. Hagenblad: Aspects of the Identification of Wiener Models. Thesis No. 793, 1999.

F. Tjärnström: Quality Estimation of Approximate Models. Thesis No. 810, 2000.

C. Carlsson: Vehicle Size and Orientation Estimation Using Geometric Fitting. Thesis No. 840, 2000.

J. Löfberg: Linear Model Predictive Control: Stability and Robustness. Thesis No. 866, 2001.

O. Härkegård: Flight Control Design Using Backstepping. Thesis No. 875, 2001.

J. Elbornsson: Equalization of Distortion in A/D Converters. Thesis No. 883, 2001.

J. Roll: Robust Verification and Identification of Piecewise Affine Systems. Thesis No. 899, 2001.

I. Lind: Regressor Selection in System Identification using ANOVA. Thesis No. 921, 2001.

R. Karlsson: Simulation Based Methods for Target Tracking. Thesis No. 930, 2002.

P.-J. Nordlund: Sequential Monte Carlo Filters and Integrated Navigation. Thesis No. 945, 2002.

M. Östring: Identification, Diagnosis, and Control of a Flexible Robot Arm. Thesis No. 948, 2002.

C. Olsson: Active Engine Vibration Isolation using Feedback Control. Thesis No. 968, 2002.

J. Jansson: Tracking and Decision Making for Automotive Collision Avoidance. Thesis No. 965, 2002.

N. Persson: Event Based Sampling with Application to Spectral Estimation. Thesis No. 981, 2002.

D. Lindgren: Subspace Selection Techniques for Classification Problems. Thesis No. 995, 2002.

E. Geijer Lundin: Uplink Load in CDMA Cellular Systems. Thesis No. 1045, 2003.

M. Enqvist: Some Results on Linear Models of Nonlinear Systems. Thesis No. 1046, 2003.

T. Schön: On Computational Methods for Nonlinear Estimation. Thesis No. 1047, 2003.

F. Gunnarsson: On Modeling and Control of Network Queue Dynamics. Thesis No. 1048, 2003.

S. Björklund: A Survey and Comparison of Time-Delay Estimation Methods in Linear Systems. Thesis No. 1061, 2003. 
M. Gerdin: Parameter Estimation in Linear Descriptor Systems. Thesis No. 1085, 2004.

A. Eidehall: An Automotive Lane Guidance System. Thesis No. 1122, 2004.

E. Wernholt: On Multivariable and Nonlinear Identification of Industrial Robots. Thesis No. 1131, 2004.

J. Gillberg: Methods for Frequency Domain Estimation of Continuous-Time Models. Thesis No. 1133, 2004.

G. Hendeby: Fundamental Estimation and Detection Limits in Linear Non-Gaussian Systems. Thesis No. 1199, 2005.

D. Axehill: Applications of Integer Quadratic Programming in Control and Communication. Thesis No. 1218, 2005.

J. Sjöberg: Some Results On Optimal Control for Nonlinear Descriptor Systems. Thesis No. 1227, 2006.

D. Törnqvist: Statistical Fault Detection with Applications to IMU Disturbances. Thesis No. 1258, 2006.

H. Tidefelt: Structural algorithms and perturbations in differential-algebraic equations. Thesis No. 1318, 2007.

S. Moberg: On Modeling and Control of Flexible Manipulators. Thesis No. 1336, 2007.

J. Wallén: On Kinematic Modelling and Iterative Learning Control of Industrial Robots. Thesis No. 1343, 2008.

J. Harju Johansson: A Structure Utilizing Inexact Primal-Dual Interior-Point Method for Analysis of Linear Differential Inclusions. Thesis No. 1367, 2008.

J. D. Hol: Pose Estimation and Calibration Algorithms for Vision and Inertial Sensors. Thesis No. 1370, 2008.

H. Ohlsson: Regression on Manifolds with Implications for System Identification. Thesis No. 1382, 2008.

D. Ankelhed: On low order controller synthesis using rational constraints. Thesis No. 1398, 2009.

P. Skoglar: Planning Methods for Aerial Exploration and Ground Target Tracking. Thesis No. 1420, 2009.

C. Lundquist: Automotive Sensor Fusion for Situation Awareness. Thesis No. 1422, 2009.

C. Lyzell: Initialization Methods for System Identification. Thesis No. 1426, 2009.

R. Falkeborn: Structure exploitation in semidefinite programming for control. Thesis No. 1430, 2010.

D. Petersson: Nonlinear Optimization Approaches to $\mathcal{H}_{2}$-Norm Based LPV Modelling and Control. Thesis No. 1453, 2010.

Z. Sjanic: Navigation and SAR Auto-focusing in a Sensor Fusion Framework. Thesis No. 1464, 2011.

K. Granström: Loop detection and extended target tracking using laser data. Thesis No. 1465, 2011.

J. Callmer: Topics in Localization and Mapping. Thesis No. 1489, 2011.

F. Lindsten: Rao-Blackwellised particle methods for inference and identification. Thesis No. 1480, 2011.

M. Skoglund: Visual Inertial Navigation and Calibration. Thesis No. 1500, 2011.

S. Khoshfetrat Pakazad: Topics in Robustness Analysis. Thesis No. 1512, 2011.

P. Axelsson: On Sensor Fusion Applied to Industrial Manipulators. Thesis No. 1511, 2011.

A. Carvalho Bittencourt: On Modeling and Diagnosis of Friction and Wear in Industrial Robots. Thesis No. 1516, 2012.

P. Rosander: Averaging level control in the presence of frequent inlet flow upsets. Thesis No. 1527, 2012. 
N. Wahlström: Localization using Magnetometers and Light Sensors. Thesis No. 1581, 2013.

R. Larsson: System Identification of Flight Mechanical Characteristics. Thesis No. 1599, 2013.

Y. Jung: Estimation of Inverse Models Applied to Power Amplifier Predistortion. Thesis No. 1605, 2013.

M. Syldatk: On Calibration of Ground Sensor Networks. Thesis No. 1611, 2013.

M. Roth: Kalman Filters for Nonlinear Systems and Heavy-Tailed Noise. Thesis No. 1613, 2013.

D. Simon: Model Predictive Control in Flight Control Design - Stability and Reference Tracking. Thesis No. 1642, 2014.

J. Dahlin: Sequential Monte Carlo for inference in nonlinear state space models. Thesis No. 1652, 2014.

M. Kok: Probabilistic modeling for positioning applications using inertial sensors. Thesis No. 1656, 2014.

J. Linder: Graybox Modelling of Ships Using Indirect Input Measurements. Thesis No. 1681, 2014.

G. Mathai: Direction of Arrival Estimation of Wideband Acoustic Wavefields in a Passive Sensing Environment. Thesis No. 1721, 2015.

I. Nielsen: On Structure Exploiting Numerical Algorithms for Model Predictive Control. Thesis No. 1727, 2015.

C. Veibäck: Tracking of Animals Using Airborne Cameras. Thesis No. 1761, 2016.

N. Evestedt: Sampling Based Motion Planning for Heavy Duty Autonomous Vehicles. Thesis No. 1762, 2016.

H. Nyqvist: On Pose Estimation in Room-Scaled Environments. Thesis No. 1765, 2016.

Y. Zhao: Position Estimation in Uncertain Radio Environments and Trajectory Learning. Thesis No. 1772, 2017. 